TRANSACTIONS OF THE

AMERICAN MATHEMATICAL SOCIETY

Volume 360, Number 9, September 2008, Pages 4929-4987

S 0002-9947(08)04495-4

Article electronically published on April 25, 2008

\title{
THE T-LIMIT AND THE RELATED GRADIENT FLOW FOR SINGULAR PERTURBATION FUNCTIONALS OF PERONA-MALIK TYPE
}

\author{
G. BELLETTINI AND G. FUSCO
}

\begin{abstract}
We consider a class of nonconvex functionals of the gradient in one dimension, which we regularize with a second order derivative term. After a proper rescaling, suggested by the associated dynamical problems, we show that the sequence $\left\{F_{\nu}\right\}$ of regularized functionals $\Gamma$-converges, as $\nu \rightarrow 0^{+}$, to a particular class of free-discontinuity functionals $\mathcal{F}$, concentrated on $S B V$ functions with finite energy and having only the jump part in the derivative. We study the singular dynamic associated with $\mathcal{F}$, using the minimizing movements method. We show that the minimizing movement starting from an initial datum with a finite number of discontinuities has jump positions fixed in space and whose number is nonincreasing with time. Moreover, there are a finite number of singular times at which there is a dropping of the number of discontinuities. In the interval between two subsequent singular times, the vector of the survived jumps is determined by the system of ODEs which expresses the $L^{2}$-gradient of the $\Gamma$-limit. Furthermore the minimizing movement turns out to be continuous with respect to the initial datum. Some properties of a minimizing movement starting from a function with an infinite number of discontinuities are also derived.
\end{abstract}

\section{INTRODUCTION}

Nonconvex functionals of the gradient of the form

$$
F(u, \Omega):=\frac{1}{2} \int_{\Omega} \phi(\nabla u) d x,
$$

where $\Omega \subset \mathbb{R}^{n}$ is a bounded domain and $\nabla u$ is the gradient of $u: \Omega \rightarrow \mathbb{R}^{m}$, arise in the mathematical modeling of several physical phenomena, for example, in nonlinear elasticity and phase transitions [40, 32, 1], 4, crystal growth [17, and in the theory of image processing [43], [41]. Due to the nonconvex character of the energy density $\phi$, the gradient dynamic associated with the functionals of the form (1.1) is not well posed. For instance if, as we do in the following, we assume

Received by the editors December 1, 2004 and, in revised form, September 22, 2006.

2000 Mathematics Subject Classification. Primary 49J45, 35B25, 74H40, 74G65.

Key words and phrases. Singular perturbations, $\Gamma$-convergence, gradient flows, minimizing movements.

The authors gratefully acknowledge the hospitality and the support of the Centro De Giorgi of the Scuola Normale Superiore di Pisa, where this paper was completed. The first author gratefully acknowledges also the hospitality and the support of the Max Planck Institute for Gravitational Physics in Golm. The authors are also grateful to the referee for some useful comments. 
$n=m=1, \Omega=I=] \alpha, \beta[$, then one is led to the parabolic equation

$$
u_{t}=\left(\phi^{\prime}\left(u_{x}\right)\right)_{x},
$$

coupled with suitable boundary and initial conditions, which for smooth enough solutions $u$ and provided that $\phi$ is smooth, takes the form $u_{t}=\phi^{\prime \prime}\left(u_{x}\right) u_{x x}$; such an equation has a forward-backward character depending on the sign of $\phi^{\prime \prime}\left(u_{x}\right)$. A consequence of the ill-posedness of (1.2) is that, in spite of some interesting attempts, 33., 45], 37, [27, 26], [36], 31, there is not yet a satisfactory notion of weak solution which can adequately describe the rich phenomenology observed in numerical experiments [28, 41]. A natural approach in this situation is to associate with (1.2) a family of regular problems depending on a small parameter $\varepsilon>0$ which are expected to approach (1.2) in the limit $\varepsilon \rightarrow 0^{+}$, and try to define solutions to (1.2) as limits as $\varepsilon \rightarrow 0^{+}$of solutions of the regularized problems, i.e.,

$$
u(x, t)=\lim _{\varepsilon \rightarrow 0^{+}} u^{\varepsilon}(x, t) .
$$

Various types of regularizations are possible, for instance: space discretization 27]; addition of a dissipative term of the form $-\varepsilon u_{x x t}$ [7, 8], 44]; approximation through a convolution kernel 18; approximation through a system of two equations [41. Following [45] and 23] we regularize by adding to $F$ a small higher order term and consider for $0<\varepsilon<1$ the family of functionals

$$
F^{\varepsilon}(u):=\frac{1}{2} \int_{I}\left(\varepsilon^{2}\left(u_{x x}\right)^{2}+\phi\left(u_{x}\right)\right) d x
$$

and the corresponding $L^{2}$-gradient system

$$
\left\{\begin{array}{l}
u_{t}=-\varepsilon^{2} u_{x x x x}+\frac{1}{2}\left(\phi^{\prime}\left(u_{x}\right)\right)_{x}, \\
+ \text { B.C. }
\end{array}\right.
$$

which is well posed. Equation (1.4) is a singular perturbation of (1.2), and therefore it is to be expected that, for small $\varepsilon>0$, the pathological character of (1.2) begins to show up in the solution $u^{\varepsilon}$ of (1.4). This behaviour is confirmed by numerical experiments [11 that indicate the existence of three distinct time scales with associated "slow time" $\theta$, "standard time" $t$, and "fast time" $\tau$, related to the original time $t$ by

$$
\left\{\begin{array}{l}
t / T_{\varepsilon}=\theta, \\
t=t, \\
t=\tau / \mathcal{T}_{\varepsilon},
\end{array}\right.
$$

where $T_{\varepsilon}, \mathcal{T}_{\varepsilon}>0$ are "constants of time" that converge to 0 as $\varepsilon \rightarrow 0^{+}$and determine the order of magnitude of the duration of typical phenomena observed in the dynamics of (1.4) for small $\varepsilon>0$. Heuristic arguments [1] suggest that $T_{\varepsilon}$ should be of order $O\left(\varepsilon^{2}\right)$. The order of magnitude of $\mathcal{T}_{\varepsilon}$ depends on the choice of $\phi$, see (1.9) and (1.8) below. In the time intervals of $O(1)$ of the slow time $\theta$ one observes the rapid formation of microstructures in $\sigma_{L, u}:=\left\{x \in I: u_{x}(x) \in \sigma_{L}^{\phi}\right\}$, $\sigma_{L}^{\phi}:=\left\{p \in \mathbb{R}: \phi^{\prime \prime}(p)<0\right\}$ (see [28]), where the initial datum $\bar{u}$ has derivative $\bar{u}_{x}(x)$ in the nonconvex region $\sigma_{L}^{\phi}$ of $\phi$. An important effect of this phenomenon is a drastic local reduction of the energy in $\sigma_{L, u}$ and one expects, as suggested by the simulations, that

$$
\lim _{\varepsilon \rightarrow 0^{+}} u^{\varepsilon}\left(\cdot, T_{\varepsilon}\right)=\bar{u}(\cdot)
$$


and at the same time

$$
\lim _{\varepsilon \rightarrow 0^{+}} F^{\varepsilon}\left(u^{\varepsilon}\left(\cdot, T_{\varepsilon}\right), \sigma_{L, u}\right)<F\left(\bar{u}, \sigma_{L, u}\right) .
$$

That is, the size of microstructures vanishes in the limit $\varepsilon \rightarrow 0^{+}$and one has (1.5), but nevertheless by (1.6) there is a discontinuity of the energy at $t=0$ in the limit $\varepsilon \rightarrow 0^{+}$. A rigorous proof of (1.5) and (1.6) would imply that the dynamics of (1.4) offers a first explicit example of the phenomenon of "fibrillation" as defined in [24]. After the formation of microstructures and the consequent reduction of the energy, the evolution of $u^{\varepsilon}$ in $\sigma_{L, u}$ is quite slow while, in time interval $t$ of $O(1)$, the evolution of $u^{\varepsilon}$ in $\sigma_{L, u}^{c}:=\left\{x \in I: \bar{u}_{x}(x) \in\left\{p \in \mathbb{R}: \phi^{\prime \prime}(p)>0\right\}\right\}$ seems to be dictated by (1.2), which in $\sigma_{L, u}^{c}$ has a forward parabolic character and therefore a heat-equation-like behaviour. The effect of this evolution is a substantial reduction of the energy also in $\sigma_{L, u}^{c}$. We remark that the different pace of evolution in regions $\sigma_{L, u}$ and $\sigma_{L, u}^{c}$ may, as actually shown by the simulations, generate singularities and even discontinuities at the interface between $\sigma_{L, u}$ and $\sigma_{L, u}^{c}$ (which actually has the rôle of a free boundary). When also the second phase of evolution is completed and the energy has been reduced to small values both in $\sigma_{L, u}$ and $\sigma_{L, u}^{c}, u^{\varepsilon}$ has $u_{x}^{\varepsilon}$ near the set of absolute minimizers of the convex envelope $\phi^{* *}$ of $\phi$ and therefore $u^{\varepsilon}$ is observed to assume a kind of piecewise linear structure which depends on the global structure of the particular $\phi$ considered. In the subsequent evolution, $u^{\varepsilon}$ keeps its approximate piecewise linear structure compatible with small values of the energy and evolves very slowly. This phase of "slow motion" is appropriately described by the fast time $\tau$ and has a "coarsening" effect, which consists in the reduction of the numbers of pseudo-linear elements that one observes in the graph of $u^{\varepsilon}$. In the limit $\varepsilon \rightarrow 0^{+}$the interval $\left[0, O\left(T_{\varepsilon}\right)\right.$ [ of first evolution is contracted to nothing while the interval of slow evolution $] O\left(1 / \mathcal{T}_{\varepsilon}\right),+\infty$ [ is displaced to $+\infty$. We remark that, while the formation of microstructures appears to be intrinsically related to the nonconvexity of $\phi$, the qualitative aspects of the phenomenon are strongly dependent on the particular regularization of the functional $F$ considered. For instance, for the regularization considered in [26, the region of interest for microstructures is the larger set $\sigma_{G, u}:=$ $\left\{x \in I: \phi\left(u_{x}(x)\right)>\phi^{* *}\left(u_{x}(x)\right)\right\}$. This difference in the dynamics in the first time scale leads, in general, to striking different evolutions in the second time scale [11, 35. A related phenomenon is that it is possible to associate with the same initial datum $\bar{u}$ infinitely many different evolutions by considering sequences $\bar{u}^{\varepsilon}$ of initial data that converge uniformly to $\bar{u}$ as $\varepsilon \rightarrow 0^{+}$. We refer to [1] for a discussion of this pathological behaviour. The rigorous mathematical justification of this complex phenomenology and in particular the study of the limit (1.3) is outside the scope of the present work. In the following we concentrate on the third time scale and consider nonconvex energy densities $\phi: \mathbb{R} \rightarrow[0,+\infty$ [ with the following properties:

(i) $\phi$ is continuous and $\phi(p)=\phi(-p)$ for any $p \in \mathbb{R}$;

(ii) $\phi$ is nondecreasing in $[0,+\infty[$;

(iii) $\phi^{-1}(0)=0$;

(iv) $\phi$ has sublinear growth at infinity, in the sense that

$$
\exists a \in\left[0,1\left[\lim _{p \rightarrow+\infty} \frac{\phi(\lambda p)}{\phi(p)}=\lambda^{a}, \quad \forall \lambda>0 .\right.\right.
$$

Examples of functions $\phi$ satisfying the above conditions are: $\phi(p)=\frac{p^{2}}{1+p^{2}}|p|^{a}$ for $a \in\left[0,1\left[, \phi(p)=|p|^{a}\right.\right.$ for $\left.a \in\right] 0,1\left[; \phi(p)=\log \left(1+p^{2}\right), \phi(p)=p^{2} /\left(1+p^{2}\right)\right.$, 
$\phi(p)=\min \left(p^{2}, 1\right)$ for $a=0$. In particular $\phi(p)=\log \left(1+p^{2}\right)$ is the choice considered by Perona-Malik in [43] in the context of image restoration, while (1.2) in the case $\phi(p)=\min \left\{p^{2}, 1\right\}$ is studied in [12 by using space discretizations. The assumption (iv) of sublinear growth of $\phi$ implies, together with (iii), that the convex envelope $\phi^{* *}$ of $\phi$ is identically equal to zero, and the extreme points of the set of values of $|p|$ where $\phi^{* *}$ assumes its minimum value are 0 and $+\infty$. Therefore, as confirmed by numerical experiments, in the late stages of the evolution, the solution $u^{\varepsilon}$ should have a staircase-like structure corresponding to small values of the energy. A simple argument indicates that the order of magnitude of the energy of a staircase-like function is $O(\nu \phi(1 / \nu))$, where $\nu=\nu(\varepsilon)$ is a small parameter related to the original parameter $\varepsilon$ by

$$
\varepsilon^{2}=\nu^{4} \phi(1 / \nu) .
$$

Therefore we introduce the rescaled energy

$$
F_{\nu}(u):=\frac{F^{\varepsilon}(u)}{\nu \phi(1 / \nu)}=\frac{1}{2} \int_{I}\left[\nu^{3}\left(u_{x x}\right)^{2}+\frac{1}{\nu \phi(1 / \nu)} \phi\left(u_{x}\right)\right] d x,
$$

and, correspondingly, we rescale time by introducing the fast time $\tau$ related to $t$ by the relation

$$
t=\frac{\tau}{\nu \phi(1 / \nu)}
$$

which transforms equation (1.4) into

$$
\left\{\begin{array}{l}
u_{\tau}=-\nu^{3} u_{x x x x}+\frac{\phi^{\prime \prime}\left(u_{x}\right) u_{x x}}{2 \nu \phi(1 / \nu)}, \\
+ \text { B.C. }
\end{array}\right.
$$

coupled with the initial condition

$$
u(\cdot, 0)=\bar{u} \quad \text { on } I \times\{\tau=0\} .
$$

Problem (1.10)-(1.11), assuming the initial datum $\bar{u}=\bar{u}_{\nu}$ is chosen to coincide with the solution $u^{\varepsilon}$ of (1.4) at time $t$ of order $O\left(\frac{1}{\nu \phi(1 / \nu)}\right)$ when the first two phases of the evolution are completed, should describe the slow motion observed in the simulations in the late stages of the dynamics for $\varepsilon \ll 1$. Therefore we assume that the initial datum $\bar{u}_{\nu}$ satisfies

$$
F_{\nu}\left(\bar{u}_{\nu}\right)<\text { const. }
$$

To justify the introduction of the new parameter $\nu=\nu(\varepsilon)$ defined by (1.8) we estimate the minimum energy for an efficient smooth approximation of a single step of size $s>0$. We let $I=] 0,1[$ and $h: \mathbb{R} \rightarrow[0,1]$ be a smooth nondecreasing function such that $h=0$ in $]-\infty,-1[$ and $h=1$ in $] 1,+\infty[$. We denote by $\eta$ a constant to be chosen later and define $u(r):=\operatorname{sh}(r /(\eta \nu))$. Then, as $\varepsilon \rightarrow 0^{+}, u$ 
converges to the step function $s H$, where $H$ is the Heaviside function. We compute

$$
\begin{aligned}
F^{\varepsilon}(u) & =\frac{1}{2} \int_{] 0,1[}\left(\frac{\varepsilon^{2}}{\nu^{3}} \frac{s^{2}}{\eta^{3}}\left(h_{x x}\right)^{2}+\eta \nu \phi\left(s h_{x} /(\eta \nu)\right)\right) d \sigma \\
& =\frac{1}{2} \nu \phi(1 / \nu)\left(\frac{s^{2}}{\eta^{3}} \int_{] 0,1[}\left(h_{x x}\right)^{2} d \sigma+\eta \int_{] 0,1[} \frac{\phi\left(s h_{x} /(\eta \nu)\right)}{\phi(1 / \nu)} d \sigma\right) \\
& \simeq \frac{1}{2} \nu \phi(1 / \nu)\left(\frac{s^{2}}{\eta^{3}} \int_{] 0,1[}\left(h_{x x}\right)^{2} d \sigma+s^{a} \eta^{1-a} \int_{] 0,1[}\left(h_{x}\right)^{a} d \sigma\right),
\end{aligned}
$$

where we used (1.8) and assumption (iv), that implies $\phi\left(s h_{x} /(\eta \nu)\right) \simeq\left(\frac{s h_{x}}{\eta}\right)^{a} \phi(1 / \nu)$. For fixed $\varepsilon$ (therefore for fixed $\nu$ ) the minimum of the right hand side of (1.13) is attained at $\eta=c s^{\frac{2-a}{4-a}}$ and it is given by $F^{\varepsilon}(u)=c_{1} \nu \phi(1 / \nu) s^{\frac{2+a}{4-a}} ; c$ and $c_{1}$ are coefficients that depend only on $a$ and $h$. This computation motivates the definition (1.8) of $\nu$ and suggests for the $\Gamma$-limit of the rescaled functional $F_{\nu}$ an expression of the form

$$
\mathcal{F}(u):=\gamma_{a} \sum_{x \in J_{u}}\left|u\left(x_{+}\right)-u\left(x_{-}\right)\right|^{r}, \quad u \in \mathcal{P}(I),
$$

where $r=\frac{2+a}{4-a}, \gamma_{a}=\inf _{h} c_{1}>0$ (cf. (2.4) $)$ and $J_{u}$ is the jump set of $u$. We observe that the abstract structure of (1.14) does not depend on the particular energy density in the class defined by (i)-(iv). We also remark that the derivation of (1.14) can be generalized to the case where, instead of the family $F^{\varepsilon}$, we consider the family of functionals $F_{n}^{\varepsilon}$ obtained by replacing $u_{x x}$ with the $n$-derivative for $n \geq 2$. In this more general situation (1.8) takes the form $\varepsilon^{2}=\nu^{2 n} \phi(1 / \nu)$ and the same argument developed above leads again to expression (1.14) with $r=\frac{2+(2 n-3) a}{2 n-a}$ for the possible $\Gamma$-limit of the rescaled functional $\frac{F_{n}^{\varepsilon}}{\nu \phi(1 / \nu)}$.

We now describe the content of the paper. The paper is divided into two parts. The main results of the first part are Theorems 4.1] and 4.4, where we characterize the $\Gamma-L^{1}(I)$-limit $\mathcal{F}$ of the sequence $\left\{F_{\nu}\right\}$ as $\nu \rightarrow 0^{+}$, and we find that the domain $\operatorname{Dom}(\mathcal{F})$ of $\mathcal{F}$ is a linear subspace $\mathcal{P}(I)$ of the $S B V(I)$ functions with vanishing absolutely continuous part of the distributional derivative, and that the $\Gamma$-limit is given by (1.14). Observe that, in general, $\mathcal{F}$ may be finite on functions with an infinite number of jumps. We remark that the Perona-Malik case corresponds to $a=0$, hence to the $\Gamma$-limit $\mathcal{F}(u):=\gamma_{0} \sum_{x \in J_{u}}\left|u\left(x_{+}\right)-u\left(x_{-}\right)\right|^{\frac{1}{2}}$.

The proofs of Theorems 4.1 and 4.4 use some ideas from [2], 39], 14] (where $\Gamma$-limits of functionals of the gradient with different scalings are studied) and it is based on: (a) a bound for the variation of a function $u$ such that $F_{\nu}(u)<C$ (cf. (3.14)); (b) a key observation that, in simple words, can be expressed by saying that $F_{\nu}(u)<C_{1}, \int_{I}\left|u_{x}\right| d x>C_{2}$, imply the existence of $\eta, \zeta>0$ independent of $\nu$, and of a subinterval $] \alpha_{\nu}, \beta_{\nu}\left[\subseteq I\right.$ such that $\beta_{\nu}-\alpha_{\nu}>\eta \nu$ and $\left|u_{x}\right|>\zeta / \nu$ on ]$\alpha_{\nu}, \beta_{\nu}\left[\right.$. This fact allows for the proof that in the limit $\nu \rightarrow 0^{+}$, the variation of $u$ is not diffused as in the Cantor-Vitali function but must concentrate on jumps and leads to the characterization of $\operatorname{Dom}(\mathcal{F})$ described above. This is a result that does not seem to follow directly from standard compactness theorems on $S B V$ functions. We conclude the first part of the paper with Theorem 4.7, which concerns the convergence of the solutions $u_{\nu}$ to (1.10), (1.11) corresponding to an initial condition $\bar{u}_{\nu}$ satisfying (1.12). We prove that given a positive number $\mathcal{T}$, there 
exists a subsequence of solutions $u_{\nu}: I \times\left[0, \mathcal{T}\left[\rightarrow \mathbb{R}\right.\right.$ converging in $L^{1}$ to a limit function $u \in B V\left(I \times\left[0, \mathcal{T}[)\right.\right.$ as $\nu \rightarrow 0^{+}$. Moreover, $u$ has the following properties:

(i) almost any $\tau$-section $u(\cdot, \tau)$ of $u$ is in the domain of $\mathcal{F}$;

(ii) the atomic part of the derivative measure of the $x$-section $u(x, \cdot)$ of $u$ is zero for almost every $x \in I$.

These rigorous results are in agreement with the numerical experiments of 28, 11 that show that in the late stages of the evolution the solutions of (1.4) assume a staircase-like structure with jumps which are fixed in space. Theorem 4.7 is the motivation for the second part of the paper. Indeed (i) is a first step toward the proof of the reasonable fact that (as also suggested by numerical simulations) the dynamics $\tau \rightarrow u(\cdot, \tau)$ defined by the limit function $u$ in Theorem 4.7 should coincide with the dynamics associated with the limit functional $\mathcal{F}$. A proof of this fact is outside the scope of the present paper; note however that in Theorem 5.4 we show that the gradient dynamics defined by the $\Gamma$-limit $\mathcal{F}$ shares with the map $\tau \rightarrow u(\cdot, \tau)$ property (ii) above; that is, the points of discontinuity do not move in space. Our discussion of the gradient flow defined by $\mathcal{F}$ is valid for any $r \in(0,1)$ and therefore covers also the general case $r=\frac{2+(2 n-3) a}{2 n-a}$ considered above. Let us denote by $\mathcal{P}_{\text {fin }}(I)$ the subspace of all functions of $\mathcal{P}(I)$ having a finite number of jumps. In Theorem 5.4 we focus on the case where the initial datum $\bar{u}$ belongs to $\mathcal{P}_{\text {fin }}(I)$. Since $\operatorname{Dom}(\mathcal{F})$ is an infinite dimensional subspace of $L^{2}(I)$, it is not clear a priori whether jumps may move in space and whether new jumps can be generated. Therefore we need a weak notion of gradient flow which is globally defined in time also past singularities. We adopt the notion of "minimizing movement" [22, 6]. We show that the minimizing movement $\tau \rightarrow \mathcal{M}(\mathcal{F}, \bar{u})(\tau)$ starting from $\bar{u}$ has the following properties:

a) in agreement with Theorem 4.7, the jump positions do not change with time;

b) the number $\mathcal{H}^{0}\left(J_{\mathcal{M}(\mathcal{F}, \bar{u})(\tau)}\right)$ of jump points is nonincreasing with time: there exist $M \leq N$ singular times $0<\tau_{1}<\cdots<\tau_{M}$ at which there is a dropping of the number of jumps, i.e., $\mathcal{H}^{0}\left(J_{\mathcal{M}(\mathcal{F}, \bar{u})\left(\tau_{h_{+}}\right)}\right)<\mathcal{H}^{0}\left(J_{\mathcal{M}(\mathcal{F}, \bar{u})\left(\tau_{h_{-}}\right)}\right)$, and for $\tau \geq \tau_{M}, \mathcal{M}(\mathcal{F}, \bar{u})(\tau)$ coincides with the average of $\bar{u}$. The behaviour of the minimizing movement $\tau \rightarrow \mathcal{M}(\mathcal{F}, \bar{u})(\tau)$ corresponds to the coarsening phenomenon observed in numerical experiments [28, 11;

c) in the interval between two subsequent singular times, the vector of the survived jumps is determined by the ODE which expresses the $L^{2}$-gradient of $\mathcal{F}$ restricted to the finite dimensional subspace defined by the survived jumps;

d) the minimizing movement is continuous with respect to the initial datum $\bar{u}$ (Theorem 6.1) in the sense that, given $\varepsilon>0$, there is $\delta>0$ such that

$$
\|\bar{v}-\bar{u}\|_{L^{2}(I)}<\delta, \quad|\mathcal{F}(\bar{v})-\mathcal{F}(\bar{u})|<\delta
$$

imply

$$
\|\mathcal{M}(\mathcal{F}, \bar{v})(\tau)-\mathcal{M}(\mathcal{F}, \bar{u})(\tau)\|_{L^{2}(I)}<\varepsilon \quad \tau \geq 0 .
$$

We remark that the conditions (1.15) do not impose an upper bound on the number of jump points of the perturbed initial condition $\bar{v}$. Therefore d) is not a consequence of the standard theorems of continuous dependence for ODE but, as we show in the proof, depends on a regularizing property of the gradient dynamics 
defined by $\mathcal{F}$. This property is expressed by an inequality that we derive in Lemma 6.3 which implies that small jump points disappear very quickly.

In Section 7 we consider the case where $\bar{u} \in \operatorname{Dom}(\mathcal{F})$ is allowed to have an infinite number of jump points. Here we are not able to show that the minimizing movement is unique, but we still prove that any generalized minimizing movement $\mathcal{G M}(\mathcal{F}, \bar{u})$ has the property that for each $\tau>0, \mathcal{G M}(\mathcal{F}, \bar{u})(\tau)$ has only a finite number of jump points. We also prove that for each $n \geq 1$ there is a $\tau_{n}$ such that $\mathcal{H}^{0}\left(J_{\mathcal{G} \mathcal{M}(\mathcal{F}, \bar{u})(\tau)}\right)<$ $n$ for $\tau>\tau_{n}$. In these computations, we need the characterization of the relaxed descending slope of $\mathcal{F}$; see Theorem 7.4

We conclude this introduction by recalling that part of the results proved in the present paper were announced in [10] and [9].

\section{Notation}

By $I=] \alpha, \beta[$ we indicate a bounded open interval of $\mathbb{R}$; if $v: I \rightarrow \mathbb{R}$ and $c \geq 0$, we set

$$
S_{c}(v):=\{x \in I:|v(x)|>c\},
$$

the $c$-superlevel of $|v|$. If $A \subseteq \mathbb{R}$ we let $1_{A}$ be the characteristic function of $A$; i.e., $1_{A}(x)=1$ if $x \in A$ and $1_{A}(x)=0$ if $x \notin A$. We indicate by $|A|$ the Lebesgue measure of $A$ and by $\bar{A}$ the closure of $A ; \mathcal{H}^{0}(A)$ denotes the number of elements of $A$. By $\mathbb{N}$ we denote the set of all positive natural numbers. If $t \in \mathbb{R}$, we set $\operatorname{sign}(t):=t /|t|$ if $t \neq 0$, and $\operatorname{sign}(0):=0$.

$B V(I)$ stands for the space of functions with bounded variation in $I$. If $u \in$ $B V(I)$ and $x \in I, u\left(x_{-}\right)$(resp. $u\left(x_{+}\right)$) is the right (resp. left) limit of $u$ at $x$. We let $J_{u}:=\left\{x \in I: u\left(x_{+}\right) \neq u\left(x_{-}\right)\right\}$be the set of jump points of $u$, which is at most countable. We indicate by $D u$ the distributional derivative of $u$, which is represented by a measure with finite total variation in $I$, i.e. $\int_{I}|D u|<+\infty$. We recall that $D u=u^{\prime}+D u^{j}+D u^{c}$, where $u^{\prime}$ (sometimes denoted also by $u_{x}$ ) is the absolutely continuous part of $D u$ with respect to the Lebesgue measure, $D u^{j}$ is the jump part of $D u$ and $D u^{c}$ is the Cantor part. Recall that $D u^{j}=$ $\sum_{x \in J_{u}}\left(u\left(x_{+}\right)-u\left(x_{-}\right)\right) \delta_{x}$, where $\delta_{x}(A)=1$ if $x \in A$ and $\delta_{x}(A)=0$ if $x \notin A$. $S B V(I)$ is the space of functions of special bounded variation in $I$, i.e., $u \in S B V(I)$ if and only if $u \in B V(I)$ and $D u^{c}=0$ in $I$. We refer to [5] for the general theory of $B V$ functions. We indicate by $\|\cdot\|_{p}$ the $L^{p}(I)$-norm, for $p \in[1,+\infty]$. We recall the following definition.

Definition 2.1. Let $\left\{F_{h}\right\}, F: L^{1}(I) \rightarrow[0,+\infty]$. We say that the sequence $\left\{F_{h}\right\}$ $\Gamma-L^{1}(I)$-converges to $F$, and we write $F=\Gamma-\lim _{h \rightarrow+\infty} F_{h}$, if the following two conditions are satisfied:

(i) for any $u \in L^{1}(I)$ and any sequence $\left\{u_{h}\right\} \subset L^{1}(I)$ converging to $u$ in $L^{1}(I)$ we have $F(u) \leq \liminf _{h \rightarrow+\infty} F_{h}\left(u_{h}\right)$;

(ii) for any $u \in L^{1}(I)$ there exists a sequence $\left\{u_{h}\right\} \subset L^{1}(I)$ converging to $u$ in $L^{1}(I)$ such that $F(u)=\lim _{h \rightarrow+\infty} F_{h}\left(u_{h}\right)$.

When $\nu \in] 0,1[$ is a continuous parameter, we say that the family of functionals $\left\{F_{\nu}\right\}_{\nu} \Gamma$-converges to $F$ as $\nu \rightarrow 0^{+}$if $\left\{F_{\nu_{h}}\right\} \Gamma$-converges to $F$ for every subsequence $\left\{\nu_{h}\right\}$ converging to zero as $h \rightarrow \infty$.

Concerning the main properties and definitions about $\Gamma$-convergence, we refer to [21], [15, [16]. 
2.1. The spaces $\mathcal{P}(l)$ and $\mathcal{P}_{\text {fin }}$. We let

$\mathcal{P}(I):=\left\{u \in S B V(I): u^{\prime}=0\right.$ in $\left.I\right\}, \quad \mathcal{P}_{\text {fin }}(I):=\left\{u \in \mathcal{P}(I): J_{u}\right.$ is a finite set $\}$.

We often represent a function $u \in \mathcal{P}(I)$ as follows: if $x \in I$, we have (see [5, Theorem 3.28, Section 4.1])

$$
u(x)=u\left(\alpha_{+}\right)+\sum_{y \in J_{u}}\left(u\left(y_{+}\right)-u\left(y_{-}\right)\right) 1_{[y, \beta[}(x), \quad x \in I,
$$

and we have chosen the right continuous representative of $u$ in its Lebesgue equivalence class. Recall that $\|u\|_{\infty} \leq\left|s_{0}\right|+\int_{I}|D u|=\left|u\left(\alpha_{+}\right)\right|+\sum_{y \in J_{u}}\left|u\left(y_{+}\right)-u\left(y_{-}\right)\right|$. Sometimes, instead of (2.2), we write

$$
u(x)=s_{0}+\sum_{i} s_{i} 1_{\left[x_{i}, \beta[\right.}(x), \quad x \in I,
$$

where, unless otherwise specified,

$$
s_{0}:=u\left(\alpha_{+}\right), \quad s_{i}:=u\left(x_{i_{+}}\right)-u\left(x_{i_{-}}\right) \neq 0, \quad J_{u}=\left\{x_{i}\right\} .
$$

When $u \in \mathcal{P}_{\text {fin }}(I)$, in (2.3) there is a finite sum, and we usually assume that $x_{i}<x_{j}$ for $i<j$.

2.2. The rescaled functionals $F_{\nu}$. Given $\nu>0$ we define the map $F_{\nu}: L^{1}(I) \rightarrow$ $[0,+\infty]$ as

$$
F_{\nu}(u):= \begin{cases}\frac{1}{2} \int_{I}\left[\nu^{3}\left(u^{\prime \prime}\right)^{2}+\frac{1}{\nu \phi(1 / \nu)} \phi\left(u^{\prime}\right)\right] d x & \text { if } u \in H^{2}(I), \\ +\infty & \text { elsewhere in } L^{1}(I) .\end{cases}
$$

If $A \subset I$ is a Borel set and $u \in H^{2}(I)$, we set

$$
F_{\nu}(u, A):=\frac{1}{2} \int_{A}\left[\nu^{3}\left(u^{\prime \prime}\right)^{2}+\frac{1}{\nu \phi(1 / \nu)} \phi\left(u^{\prime}\right)\right] d x .
$$

Remark 2.2. Observe that $F_{\nu}$ enjoys the following scaling property: if $u \in H^{2}(I)$ and if we define $u_{\nu}$ by $u_{\nu}(y)=: u(\nu y)$ for any $y \in I / \nu$, then $u_{\nu} \in H^{2}(I / \nu)$ and

$$
F_{\nu}(u)=\frac{1}{2} \int_{I / \nu}\left[\left(u_{\nu}^{\prime \prime}\right)^{2}+\frac{\phi\left(u_{\nu}^{\prime} / \nu\right)}{\phi(1 / \nu)}\right] d y .
$$

2.3. The limit functional $\mathcal{F}$. The functional $\mathcal{F}: L^{1}(I) \rightarrow[0,+\infty]$ is defined as follows:

$$
\mathcal{F}(u):= \begin{cases}\gamma_{a} \sum_{x \in J_{u}}\left|u\left(x_{+}\right)-u\left(x_{-}\right)\right|^{\frac{2+a}{-a}} & \text { if } u \in \mathcal{P}(I), \\ +\infty & \text { elsewhere in } L^{1}(I) .\end{cases}
$$

Here the constant $\gamma_{a}$ will turn out to have the following expression:

$$
\begin{aligned}
\gamma_{a}:=\frac{1}{2}\left[\left(\frac{3}{1-a}\right)^{\frac{1-a}{4-a}}+\left(\frac{1-a}{3}\right)^{\frac{3}{4-a}}\right] \\
\quad \times \inf _{\widehat{\psi} \in \widehat{Y}}\left\{\left(\int_{] 0,1[}\left|\widehat{\psi}^{\prime}\right|^{a} d x\right)^{\frac{3}{4-a}}\left(\int_{] 0,1[}\left|\widehat{\psi}^{\prime \prime}\right|^{2} d x\right)^{\frac{1-a}{4-a}}\right\},
\end{aligned}
$$


where

$$
\widehat{Y}:=\left\{\widehat{\psi} \in H^{2}(] 0,1[): \widehat{\psi}(0)=0, \widehat{\psi}(1)=1, \widehat{\psi}^{\prime}(0)=\widehat{\psi}^{\prime}(1)=0\right\} .
$$

Observe that, if we represent $u \in \mathcal{P}(I)$ as in (2.3), then $\mathcal{F}(u)=\gamma_{a} \sum_{i}\left|s_{i}\right|^{\frac{2+a}{4-a}}$.

Note that $\mathcal{F}$ can be written, for $u \in S B V(I)$, as

$$
\mathcal{F}(u)=\int_{I} \chi_{\mathbb{R} \backslash\{0\}}\left(u^{\prime}\right) d x+\gamma_{a} \sum_{x \in J_{u}}\left|u\left(x_{+}\right)-u\left(x_{-}\right)\right|^{\frac{2+a}{4-a}},
$$

where $\chi_{\mathbb{R} \backslash\{0\}}(p):=+\infty$ if $p \neq 0$ and $\chi_{\mathbb{R} \backslash\{0\}}(0)=0$.

Remark 2.3. When $a=0$ we have $\frac{2+a}{4-a}=1 / 2$ and $\gamma_{0}=2 \sqrt{2 / 3}$.

In the following we often use the notation

$$
\mathrm{e}_{a}(s):=\gamma_{a}|s|^{r}, \quad r:=\frac{2+a}{4-a} .
$$

We set

$$
\operatorname{Dom}(\mathcal{F}):=\left\{u \in L^{1}(I): \mathcal{F}(u)<+\infty\right\}
$$

In the sequel we will use the following inequality, valid for any sequence of real numbers $\left\{x_{i}\right\}$ and $\left.\left.\alpha \in\right] 0,1\right]$ :

$$
\sum_{i=1}^{\infty}\left|x_{i}\right| \leq\left(\sum_{i=1}^{\infty}\left|x_{i}\right|^{\alpha}\right)^{1 / \alpha}
$$

Indeed, if the right hand side of (2.6) is finite, given $N$ and letting $|\bar{x}|:=\max \left\{\left|x_{1}\right|\right.$, $\left.\ldots,\left|x_{N}\right|\right\}$, we have

$$
\sum_{i=1}^{N}\left|x_{i}\right|=|\bar{x}| \sum_{i=1}^{N} \frac{\left|x_{i}\right|}{|\bar{x}|} \leq|\bar{x}| \sum_{i=1}^{N}\left(\frac{\left|x_{i}\right|}{|\bar{x}|}\right)^{\alpha}=|\bar{x}|^{1-\alpha} \sum_{i=1}^{N}\left|x_{i}\right|^{\alpha} \leq\left(\sum_{i=1}^{N}\left|x_{i}\right|\right)^{1-\alpha} \sum_{i=1}^{N}\left|x_{i}\right|^{\alpha},
$$

which implies (2.6).

Remark 2.4. Observe that:

(a) $\mathcal{P}_{\text {fin }}(I) \subsetneq \operatorname{Dom}(\mathcal{F}) \subsetneq \mathcal{P}(I)$

(b) If $u \in \operatorname{Dom}(\mathcal{F})$, we have

$$
\|u\|_{\infty} \leq\left|s_{0}\right|+\int_{I}|D u|=\left|s_{0}\right|+\sum_{i}\left|s_{i}\right| \leq\left|s_{0}\right|+\left(\frac{\mathcal{F}(u)}{\gamma_{a}}\right)^{1 / r} .
$$

\section{Preliminary lemmata}

Lemma 3.1. For any $\delta \in] 0,1\left[\right.$ there exists $r_{\delta}>0$ such that

$$
(1-\delta)\left(\frac{p}{q}\right)^{a(1-\varrho \delta)} \leq \frac{\phi(p)}{\phi(q)} \leq(1-\delta)^{-1}\left(\frac{p}{q}\right)^{a(1+\varrho \delta)}, \quad \forall p, q \geq r_{\delta},
$$

where $\varrho:=\operatorname{sign}(p-q)$.

Proof. If $a=0$, inequalities (3.1) follow directly from (1.7). Hence we suppose $a \in] 0,1[$. Let us assume first that $p>q$. Fix $\Lambda \in] 1, p / q[$ and $\eta \in] 0, \Lambda^{a}[$ such that

$$
\frac{\log \left(\frac{\Lambda^{a}+\eta}{\Lambda^{a}}\right)}{\log \Lambda}<\delta a, \quad \Lambda^{a}+\eta<(1-\delta)^{-1}
$$


and

$$
\frac{\log \left(\frac{\Lambda^{a}-\eta}{\Lambda^{a}}\right)}{\log \Lambda}>-\delta a, \quad \frac{1}{\Lambda^{a}}>1-\delta .
$$

Using (1.7), let $r_{\eta, \Lambda}$ be such that

$$
\Lambda^{a}-\eta \leq \frac{\phi(\Lambda q)}{\phi(q)} \leq \Lambda^{a}+\eta, \quad \forall q \geq r_{\eta, \Lambda} .
$$

Let $k>0$ be such that $\frac{\log (p / q)}{\log \Lambda} \in[k, k+1]$, i.e.,

$$
\Lambda^{k} q \leq p \leq \Lambda^{k+1} q .
$$

Iterating (3.4), it follows

$$
\left(\Lambda^{a}-\eta\right)^{k} \leq \frac{\phi\left(\Lambda^{k} q\right)}{\phi(q)} \leq\left(\Lambda^{a}+\eta\right)^{k}, \quad \forall q \geq r_{\eta, \Lambda} .
$$

Using (3.5) and recalling that $\phi$ is nondecreasing in $[0,+\infty$ [ we get

$$
\left(\Lambda^{a}-\eta\right)^{k} \leq \frac{\phi\left(\Lambda^{k} q\right)}{\phi(q)} \leq \frac{\phi(p)}{\phi(q)} \leq \frac{\phi\left(\Lambda^{k+1} q\right)}{\phi(q)} \leq\left(\Lambda^{a}+\eta\right)^{k+1}, \quad \forall q \geq r_{\eta, \Lambda} .
$$

Observe now that, using (3.5),

$$
\left(\Lambda^{a}+\eta\right)^{k+1}=\left(\frac{\Lambda^{a}+\eta}{\Lambda^{a}}\right)^{k+1} \Lambda^{a} \Lambda^{k a} \leq\left(\frac{\Lambda^{a}+\eta}{\Lambda^{a}}\right)^{k+1} \Lambda^{a}\left(\frac{p}{q}\right)^{a} .
$$

Since $k \leq \frac{\log \left(\frac{p}{q}\right)}{\log \Lambda}$, by (3.8) we deduce

$$
\left(\Lambda^{a}+\eta\right)^{k+1} \leq\left(\Lambda^{a}+\eta\right)\left(\frac{\Lambda^{a}+\eta}{\Lambda^{a}}\right)^{\frac{\log \frac{p}{q}}{\log \Lambda}}\left(\frac{p}{q}\right)^{a}=\left(\Lambda^{a}+\eta\right)\left(\frac{p}{q}\right)^{a+\left(\frac{\log \left(\frac{\Lambda^{a}+\eta}{\Lambda^{a}}\right)}{\log \Lambda}\right)} .
$$

Therefore, coupling (3.7), (3.9) and (3.2), we obtain

$$
\frac{\phi(p)}{\phi(q)} \leq\left(\Lambda^{a}+\eta\right)\left(\frac{p}{q}\right)^{a+\left(\frac{\log \left(\frac{\Lambda^{a}+\eta}{\Lambda^{a}}\right)}{\log \Lambda}\right)}<(1-\delta)^{-1}\left(\frac{p}{q}\right)^{a(1+\delta)}
$$

which is the right inequality in (3.1).

The left inequality for $p>q$ in (3.1) can be proved as follows. Using (3.5) and (3.3) we have

$$
\begin{aligned}
\left(\Lambda^{a}-\eta\right)^{k} & =\left(\frac{\Lambda^{a}-\eta}{\Lambda^{a}}\right)^{k} \frac{1}{\Lambda^{a}} \Lambda^{(k+1) a} \geq\left(\frac{\Lambda^{a}-\eta}{\Lambda^{a}}\right)^{k} \frac{1}{\Lambda^{a}}\left(\frac{p}{q}\right)^{a} \\
& \geq \frac{1}{\Lambda^{a}}\left(\frac{\Lambda^{a}-\eta}{\Lambda^{a}}\right)^{\frac{\log \left(\frac{p}{q}\right)}{\log \Lambda}}\left(\frac{p}{q}\right)^{a}=\frac{1}{\Lambda^{a}}\left(\frac{p}{q}\right)^{a+\left(\frac{\log \left(\frac{\Lambda^{a}-\eta}{\Lambda^{a}}\right)}{\log \Lambda}\right)} \\
& >(1-\delta)\left(\frac{p}{q}\right)^{a(1-\delta)} .
\end{aligned}
$$

Assume now $p \leq q$. From (3.1) we have

$$
(1-\delta)\left(\frac{q}{p}\right)^{a(1-\hat{\varrho} \delta)} \leq \frac{\phi(q)}{\phi(p)} \leq(1-\delta)^{-1}\left(\frac{q}{p}\right)^{a(1+\hat{\varrho} \delta)}, \quad \forall p, q>r_{\delta},
$$


where $\hat{\varrho}:=\operatorname{sign}(q-p)$. Hence

$$
\begin{aligned}
(1-\delta)\left(\frac{p}{q}\right)^{a(1+\hat{\varrho} \delta)} & =(1-\delta)\left(\frac{p}{q}\right)^{a(1-\varrho \delta)} \leq \frac{\phi(p)}{\phi(q)} \leq(1-\delta)^{-1}\left(\frac{p}{q}\right)^{a(1-\hat{\varrho} \delta)} \\
& =(1-\delta)^{-1}\left(\frac{p}{q}\right)^{a(1+\varrho \delta)}
\end{aligned}
$$

for $p, q>r_{\delta}$, and the assertion follows.

In the following lemma we give a bound for the $L^{\infty}(I)$ and the $L^{1}(I)$ norms of the derivative of a function $u$ in $H^{2}(I)$ in terms of its energy $F_{\nu}(u)$.

Lemma 3.2. Let $u \in H^{2}(I)$ and $\left.\left.\nu \in\right] 0,1\right]$. Then

$$
\left|S_{\alpha}\left(u^{\prime}\right)\right| \leq \frac{2}{\phi(\alpha)} \nu \phi(1 / \nu) F_{\nu}(u) \quad \forall \alpha>0 .
$$

Moreover, there exist $\bar{\nu}>0$ and a decreasing function $\omega:] 0, \bar{\nu}[\rightarrow] 0,+\infty[$ with $\lim _{s \rightarrow 0^{+}} \omega(s)=0$, such that

$$
\left.\left\|u^{\prime}\right\|_{\infty} \leq \frac{1}{\nu}\left(1+2 F_{\nu}(u)\right), \quad \nu \in\right] 0, \bar{\nu}[
$$

and

$$
\left.\int_{I}\left|u^{\prime}\right| d x \leq C\left(\omega(\nu)+F_{\nu}(u)\right), \quad \nu \in\right] 0, \bar{\nu}[.
$$

Proof. Recalling the definition of $S_{\alpha}\left(u^{\prime}\right)$ in (2.1) and that $\phi$ is even and nondecreasing, we have

$$
F_{\nu}(u) \geq \frac{1}{2} \int_{S_{\alpha}\left(u^{\prime}\right)} \frac{\phi\left(u^{\prime}\right)}{\nu \phi(1 / \nu)} d x \geq \frac{\left|S_{\alpha}\left(u^{\prime}\right)\right| \phi(\alpha)}{2 \nu \phi(1 / \nu)},
$$

and (3.12) follows.

Write $S_{\frac{1}{\nu}}\left(u^{\prime}\right)$ as the union of its connected components, i.e., $\left.S_{\frac{1}{\nu}}\left(u^{\prime}\right)=\bigcup_{k}\right] a_{\nu}^{k}, b_{\nu}^{k}[$. Arguing exactly as in [39, Lemma 3.8] and using (3.12) we get, for $y \in] a_{\nu}^{k}, b_{\nu}^{k}[$,

$$
\begin{aligned}
\left|u^{\prime}(y)\right| & \leq\left|u^{\prime}\left(a_{\nu}^{k}\right)\right|+\int_{] a_{\nu}^{k}, y[}\left|u^{\prime \prime}\right| d x \leq \frac{1}{\nu}+\frac{\left|S_{\frac{1}{\nu}}\left(u^{\prime}\right)\right|^{1 / 2}}{\nu^{3 / 2}}\left(\nu^{3} \int_{] a_{\nu}^{k}, b_{\nu}^{k}[}\left|u^{\prime \prime}\right|^{2} d x\right)^{1 / 2} \\
& \leq \frac{1}{\nu}+\frac{\left|S_{\frac{1}{\nu}}\left(u^{\prime}\right)\right|^{1 / 2}}{\nu^{3 / 2}}\left(2 F_{\nu}(u)\right)^{1 / 2} \leq \frac{1}{\nu}+\frac{2 \nu^{1 / 2}}{\nu^{3 / 2}} F_{\nu}(u)=\frac{1}{\nu}\left(1+2 F_{\nu}(u)\right)
\end{aligned}
$$

and (3.13) follows.

It remains to prove (3.14). Define the increasing sequence $\left\{\alpha_{k}(\nu)\right\}_{k} \subset[0,+\infty[$ as follows: $\alpha_{0}(\nu):=0, \alpha_{1}(\nu):=\max \left\{\phi^{-1}(\nu(\phi(1 / \nu)))\right\}, \alpha_{2}(\nu):=1$, and

$$
\alpha_{k}(\nu):=\frac{k}{\nu \phi(1 / \nu)}, \quad k \geq 3,
$$

where $\nu>0$ is small enough in such a way that $\alpha_{1}(\nu)<1$. In the remaining part of the proof we simply write $S_{\lambda}$ instead of $S_{\lambda}\left(u^{\prime}\right)$, and $\alpha_{k}$ instead of $\alpha_{k}(\nu)$. Set

$$
N_{\nu}:=\left[\left(1+2 F_{\nu}(u)\right) \phi(1 / \nu)\right],
$$


[.] denoting the integer part. From (3.13), we have $S_{\alpha_{k}}=\emptyset$ for all $k>N_{\nu}$. Hence we can write

$$
\begin{aligned}
\int_{I}\left|u^{\prime}\right| d x & =\sum_{k=1}^{N_{\nu}} \int_{S_{\alpha_{k-1}} \backslash S_{\alpha_{k}}}\left|u^{\prime}\right| d x \leq \sum_{k=1}^{N_{\nu}} \alpha_{k}\left(\left|S_{\alpha_{k-1}}\right|-\left|S_{\alpha_{k}}\right|\right) \\
& =\sum_{k=1}^{N_{\nu}} \alpha_{k}\left|S_{\alpha_{k-1}}\right|-\sum_{k=2}^{N_{\nu}+1} \alpha_{k-1}\left|S_{\alpha_{k-1}}\right| \\
& =\alpha_{1}\left|S_{\alpha_{0}}\right|+\left(\alpha_{2}-\alpha_{1}\right)\left|S_{\alpha_{1}}\right|+\sum_{k=3}^{N_{\nu}}\left(\alpha_{k}-\alpha_{k-1}\right)\left|S_{\alpha_{k-1}}\right|-\alpha_{N_{\nu}}\left|S_{\alpha_{N_{\nu}}}\right| \\
& \leq \alpha_{1}|I|+\alpha_{2}\left|S_{\alpha_{1}}\right|+\alpha_{3}\left|S_{\alpha_{2}}\right|+\sum_{k=4}^{N_{\nu}}\left(\alpha_{k}-\alpha_{k-1}\right)\left|S_{\alpha_{k-1}}\right| .
\end{aligned}
$$

Hence, using (3.12) and the equality $\alpha_{k}-\alpha_{k-1}=\frac{1}{\nu \phi(1 / \nu)}, k \geq 4$, we deduce

$$
\int_{I}\left|u^{\prime}\right| d x \leq \alpha_{1}|I|+2 F_{\nu}(u)+\frac{6}{\phi(1)} F_{\nu}(u)+2 F_{\nu}(u) \sum_{k=4}^{N_{\nu}} \frac{1}{\phi\left(\frac{k-1}{\nu \phi\left(\frac{1}{\nu}\right)}\right)} .
$$

We now prove that the sum on the right hand side of (3.16) is uniformly bounded with respect to $\nu$, provided $\nu>0$ is sufficiently small. Recalling also that $\phi$ is nondecreasing and the definition of $N_{\nu}$ in (3.15) we have

$$
\sum_{k=4}^{N_{\nu}} \frac{1}{\phi\left(\frac{k-1}{\nu \phi\left(\frac{1}{\nu}\right)}\right)}=\sum_{k=3}^{N_{\nu}-1} \int_{k}^{k+1} \frac{1}{\phi\left(\frac{k}{\nu \phi\left(\frac{1}{\nu}\right)}\right)} d s \leq \nu \int_{\frac{3}{\nu \phi(1 / \nu)}}^{\frac{C}{\nu}} \frac{\phi(1 / \nu)}{\phi(s)} d s,
$$

where we have set $C:=\left[1+2 F_{\nu}(u)\right]$. Fix $\left.\delta \in\right] 0,1[$ in such a way that $a(1+\delta)<1$. Then Lemma 3.1 and the change of variable $\nu s=\tau$ imply

$$
\nu \int_{\frac{3}{\nu \phi(1 / \nu)}}^{\frac{C}{\nu}} \frac{\phi(1 / \nu)}{\phi(s)} d s \leq(1-\delta)^{-1} \int_{\frac{3}{\phi(1 / \nu)}}^{C} \frac{d \tau}{\tau^{a(1+\varrho(\tau) \delta)}}
$$

where $\varrho(\tau):=\operatorname{sign}\left(\frac{1}{\nu}-\frac{\tau}{\nu}\right)$. The last integral is bounded by a constant independent of $\nu$, since

$$
\int_{\frac{3}{\phi(1 / \nu)}}^{C} \frac{d \tau}{\tau^{a(1+\varrho(\tau) \delta)}} \leq \int_{0}^{C}\left(\frac{1}{\tau^{a(1-\delta)}}+\frac{1}{\tau^{a(1+\delta)}}\right) d \tau
$$

Hence estimate (3.14) follows.

Remark 3.3. In the proof of Lemma 3.2 we have actually proved an assertion stronger than (3.13), namely $\left\{\left|u^{\prime}\right|>1 / \nu\right\} \subseteq\left\{\left|u^{\prime}\right| \leq \frac{1}{\nu}\left(1+2 F_{\nu}(u)\right)\right\}$.

\section{Equicoerciveness and $\Gamma$-COnVERGence}

A key step toward the characterization of the $\Gamma-L^{1}(I)$-limit of the sequence $\left\{F_{\nu}\right\}$ is the following compactness result.

Theorem 4.1. Let $\left\{u_{\nu}\right\} \subset H^{2}(I)$ be a sequence satisfying

$$
\sup _{\nu} F_{\nu}\left(u_{\nu}\right)<+\infty
$$


and such that $\int_{I} u_{\nu} d x=0$ for any $\nu$. Then there exist a subsequence (still denoted by $\left\{u_{\nu}\right\}$ ) and a function $u \in \mathcal{P}(I)$ such that $\left\{u_{\nu}\right\}$ weakly* converges to $u$ in $B V(I)$ as $\nu \rightarrow 0^{+}$.

Remark 4.2. For the compactness result in Theorem 4.1 some assumption on $u_{\nu_{k}}$ is necessary, because $F_{\nu}$ depends on $u^{\prime}$ and $u^{\prime \prime}$, but does not depend explicitly on $u$. The condition $\int_{I} u_{\nu} d x=0$ is quite natural when we observe that the integral of $u$ is conserved along solutions of (1.10), (1.11).

Proof. By (3.14), (4.1) and $\int_{I} u_{\nu} d x=0$, we have that $\sup _{\nu}\left\|u_{\nu}\right\|_{B V(I)}<+\infty$. Hence [5] there exist a subsequence (still denoted by $\left\{u_{\nu}\right\}$ ) and a function $u \in$ $B V(I)$ such that $\left\{u_{\nu}\right\}$ converges pointwise almost everywhere and weakly* in $B V(I)$ to $u$ as $\nu \rightarrow 0^{+}$.

It remains to prove that $u \in \mathcal{P}(I)$. We can assume that $\int_{I}|D u|>0$; otherwise there is nothing to prove. Recall [5] that

$$
\int_{I}|D u| \leq \liminf _{\nu \rightarrow 0} \int_{I}\left|u_{\nu}^{\prime}\right| d x
$$

Let

$$
E:=\sup _{\nu} F_{\nu}\left(u_{\nu}\right)<+\infty
$$

We divide the proof into five steps. Set $S_{c}^{\nu}=S_{c}\left(u_{\nu}^{\prime}\right)$ and $S_{c(\nu)}=S_{c(\nu)}^{\nu}$. Let $\left.\delta \in\right] 0,1[$ be such that

$$
\delta a<\frac{1-a}{2}=: \theta
$$

(in particular $a(1+\delta)<1$ ). Let $r_{\delta}$ be as in Lemma 3.1

Step 1. There exist $\nu_{1}>0$ and a positive nondecreasing function $\left.\left.\omega_{1}:\right] 0, \nu_{1}\right] \rightarrow$ ] $0,+\infty\left[\right.$ with $\lim _{\nu \rightarrow 0^{+}} \omega_{1}(\nu)=0$, such that

$$
\left.\int_{I \backslash S_{\frac{1}{\nu^{\theta}}}}\left|u_{\nu}^{\prime}\right| d x \leq \omega_{1}(\nu), \quad \nu \in\right] 0, \nu_{1}[.
$$

Set $\gamma(\nu):=\max \left\{\phi^{-1}\left(\nu^{\theta}\right)\right\}>0$. We have

$$
\begin{aligned}
\int_{I \backslash S_{\frac{1}{\nu^{\theta}}}}\left|u_{\nu}^{\prime}\right| d x \leq & \int_{\left\{0<\left|u_{\nu}^{\prime}\right| \leq \gamma(\nu)\right\}}\left|u_{\nu}^{\prime}\right| d x+\int_{\left\{\gamma(\nu)<\left|u_{\nu}^{\prime}\right| \leq 1\right\}}\left|u_{\nu}^{\prime}\right| d x \\
& +\int_{\left\{1<\left|u_{\nu}^{\prime}\right| \leq \frac{1}{\nu^{\theta / 2}}\right\}}\left|u_{\nu}^{\prime}\right| d x+\int_{\left\{\frac{1}{\nu^{\theta / 2}}<\left|u_{\nu}^{\prime}\right| \leq \frac{1}{\nu^{\theta}}\right\}}\left|u_{\nu}^{\prime}\right| d x \\
\leq & \gamma(\nu)|I|+\left|S_{\gamma(\nu)}\right|+\frac{1}{\nu^{\theta / 2}}\left|S_{1}\right|+\frac{1}{\nu^{\theta}}\left|S_{\frac{1}{\nu^{\theta / 2}}}\right| .
\end{aligned}
$$


Using (3.12), the definition of $\gamma(\nu)$, (3.1), (4.3), and setting $C_{\delta}:=\frac{\phi\left(r_{\delta}\right)}{r_{\delta}^{a(1+\delta)}}$, we have

$$
\begin{aligned}
\left|S_{\gamma(\nu)}\right| & \leq 2 E \frac{\nu \phi(1 / \nu)}{\phi(\gamma(\nu))}=2 E \nu^{1-\theta} \phi(1 / \nu) \leq 2 E C_{\delta}(1-\delta)^{-1} \nu^{1-a-\theta-\delta a} \leq 4 E C_{\delta} \nu^{\theta-\delta a}, \\
\frac{1}{\nu^{\theta / 2}}\left|S_{1}\right| & \leq \frac{2 E}{\phi(1)} \nu^{1-\frac{\theta}{2}} \phi(1 / \nu) \leq \frac{4 E}{\phi(1)} C_{\delta} \nu^{1-\frac{\theta}{2}-a-\delta a}=\frac{4 E}{\phi(1)} C_{\delta} \nu^{\frac{3 \theta}{2}-\delta a}, \\
\frac{1}{\nu^{\theta}}\left|S_{\frac{1}{\nu^{\theta / 2}}}\right| & \leq 2 E \nu^{1-\theta} \frac{\phi(1 / \nu)}{\phi\left(1 / \nu^{\theta / 2}\right)} \leq 4 E \nu^{1-\theta-a+a \frac{\theta}{2}-\delta a\left(1-\frac{\theta}{2}\right)} \\
& =4 E \nu^{\theta\left(\frac{3}{2}-\theta\right)-\delta a\left(1-\frac{\theta}{2}\right)} \leq 4 E \nu^{\theta-\delta a} .
\end{aligned}
$$

Then step 1 follows with

$$
\omega_{1}(\nu):=\gamma(\nu)|I|+4 E C_{\delta} \nu^{\theta-\delta a}+\frac{4 E}{\phi(1)} C_{\delta} \nu^{\frac{3}{2} \theta-\delta a}+4 E \nu^{\theta-\delta a} .
$$

Write $S_{\frac{1}{\nu^{\theta}}}$ as the union of its (open) connected components, i.e.,

$$
\left.S_{\frac{1}{\nu^{\theta}}}=\bigcup_{j \geq 1}\right] \alpha_{j}, \beta_{j}[\text {. }
$$

Define

$$
\left\{\begin{array}{l}
\eta_{j}:=\frac{\beta_{j}-\alpha_{j}}{\nu} \\
w_{j}(x):=u_{\nu}(x)-u_{\nu}\left(\alpha_{j}\right)-u_{\nu}^{\prime}\left(\alpha_{j}\right)\left(x-\alpha_{j}\right), \quad x \in\left[\alpha_{j}, \beta_{j}\right] \\
\zeta_{j}:=w_{j}\left(\beta_{j}\right) .
\end{array}\right.
$$

Observe that $\alpha_{j}, \beta_{j}, \eta_{j}, w_{j}, \zeta_{j}$ depend on $\nu$, and $w_{j}(x)=u_{\nu}(x)-u_{\nu}\left(\alpha_{j}\right)-$ $\operatorname{sign}\left(u_{\nu}^{\prime}\left(\alpha_{j}\right)\right) \frac{1}{\nu^{\theta}}\left(x-\alpha_{j}\right)$ for $x \in\left[\alpha_{j}, \beta_{j}\right]$ (see Figure 1). Without loss of generality, we can assume that

$$
\left|\zeta_{j+1}\right| \leq\left|\zeta_{j}\right| \quad \forall j, \forall \nu
$$

Step 2. There exist $\nu_{2}>0$ and a positive nondecreasing function $\left.\omega_{2}:\right] 0, \nu_{2}[\rightarrow$ ] $0,+\infty\left[\right.$ with $\lim _{\nu \rightarrow 0^{+}} \omega_{2}(\nu)=0$, such that

$$
\left.\sum_{j}\left|\zeta_{j}\right| \geq \int_{I}|D u|-\omega_{2}(\nu), \quad \nu \in\right] 0, \nu_{2}[.
$$

Recalling (3.12) we have

$$
\nu \sum_{j} \eta_{j}=\left|S_{\frac{1}{\nu^{\theta}}}\right| \leq 2 E \nu \frac{\phi(1 / \nu)}{\phi\left(1 / \nu^{\theta}\right)} ;
$$

hence from (3.1),

$$
\sum_{j} \eta_{j} \leq 4 E \frac{1}{\nu^{a(1+\delta)(1-\theta)}}
$$

provided $\nu>0$ is small enough. Observe now that

$$
\zeta_{j}=\int_{] \alpha_{j}, \beta_{j}[}\left(u_{\nu}^{\prime}-\operatorname{sign}\left(u_{\nu}^{\prime}\left(\alpha_{j}\right)\right) \frac{1}{\nu^{\theta}}\right) d x=\operatorname{sign}\left(u_{\nu}^{\prime}\left(\alpha_{j}\right)\right)\left(\int_{] \alpha_{j}, \beta_{j}[}\left|u_{\nu}^{\prime}\right| d x-\nu^{1-\theta} \eta_{j}\right)
$$




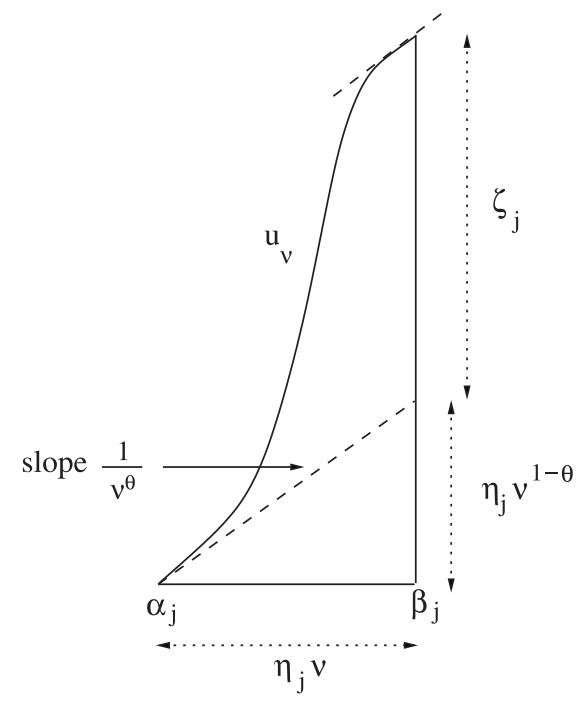

FiguRE $1 . \zeta_{j}$ is the difference between the value of $u_{\nu}$ and the linear function with slope $\frac{1}{\nu^{\theta}}$, at the right extremum $\beta_{j}$ of the interval. The two dotted lines are parallel.

so that

$$
\left|\zeta_{j}\right| \geq \int_{] \alpha_{j}, \beta_{j}[}\left|u_{\nu}^{\prime}\right| d x-\nu^{1-\theta} \eta_{j}
$$

Using (4.13) it follows that for $\nu>0$ small enough,

$$
\begin{aligned}
\sum_{j}\left|\zeta_{j}\right| & \geq \int_{S_{\frac{1}{\nu^{\theta}}}}\left|u_{\nu}^{\prime}\right| d x-\nu^{1-\theta} \sum_{j} \eta_{j} \\
& =\int_{I}\left|u_{\nu}^{\prime}\right| d x-\int_{I \backslash S_{\frac{1}{\nu^{\theta}}}}\left|u_{\nu}^{\prime}\right| d x-\nu^{1-\theta} \sum_{j} \eta_{j} \\
& \geq \int_{I}|D u|-\int_{I \backslash S_{\frac{1}{\nu^{\theta}}}}\left|u_{\nu}^{\prime}\right| d x-\lambda_{\nu}-\nu^{1-\theta} \sum_{j} \eta_{j},
\end{aligned}
$$

where in the last inequality we used (4.2) and $\left\{\lambda_{\nu}\right\}_{\nu}$ converges to zero as $\nu \rightarrow 0^{+}$. Hence, using (4.11) we deduce

$$
\sum_{j}\left|\zeta_{j}\right| \geq \int_{I}|D u|-\left(\omega_{1}(\nu)+4 E \nu^{(1-a(1+\delta))(1-\theta)}\right)-\lambda_{\nu}
$$

which proves (4.9) with $\omega_{2}(\nu):=\left(\omega_{1}(\nu)+4 E \nu^{(1-a(1+\delta))(1-\theta)}\right)-\lambda_{\nu}$.

Before moving to the next step, we introduce some notation. Following also [2, Remark 3.2], 39, Section 3], for any $s \geq 0$ we define

$$
\mathrm{e}_{a}(s):=\frac{1}{2} \inf \left\{\int_{] 0, \eta[}\left|\psi^{\prime \prime}\right|^{2} d x+\int_{] 0, \eta[}\left|\psi^{\prime}\right|^{a} d x: \eta>0, \psi \in Y_{\eta}(s)\right\},
$$


where

$$
Y_{\eta}(s):=\left\{\psi \in H^{2}(] 0, \eta[): \psi(0)=0, \psi(\eta)=s, \psi^{\prime}(0)=\psi^{\prime}(\eta)=0\right\} .
$$

Making the change of variable $\psi(x)=s \widehat{\psi}(x / \eta)$ we have

$$
\mathrm{e}_{a}(s)=\frac{1}{2} \inf _{\hat{\psi} \in \widehat{Y}} \inf _{\eta>0}\left\{\frac{s^{2}}{\eta^{3}} \int_{] 0,1[}\left|\widehat{\psi}^{\prime \prime}\right|^{2} d x+s^{a} \eta^{1-a} \int_{] 0,1[}\left|\widehat{\psi}^{\prime}\right|^{a} d x\right\},
$$

where $\widehat{Y}$ is defined in (2.5). For a fixed $\widehat{\psi}$, the infimum with respect to $\eta$ in (4.16) is attained at

$$
\eta_{\min }=\left(\frac{3 \int_{] 0,1[}\left|\widehat{\psi}^{\prime \prime}\right|^{2} d x}{(1-a) \int_{] 0,1[}\left|\widehat{\psi}^{\prime}\right|^{a} d x}\right)^{\frac{1}{4-a}} s^{\frac{2-a}{4-a}}
$$

Hence

$$
\mathrm{e}_{a}(s)=\gamma_{a} s^{\frac{2+a}{4-a}}
$$

and $\gamma_{a}$ is defined in (2.4).

Observe that when $a=0$ we have

$$
\begin{aligned}
\mathrm{e}_{0}(s) & =\frac{1}{2} \inf _{\eta>0}\left\{\eta+\inf \left\{\int_{] 0, \eta[}\left|\psi^{\prime \prime}\right|^{2} d x: \psi \in Y_{\eta}(s)\right\}\right\} \\
& =\frac{1}{2} \inf _{\eta>0}\left\{\eta+s^{2} \eta^{-3} c\right\}=\gamma_{0} \sqrt{s}
\end{aligned}
$$

where $c=\inf \left\{\int_{] 0,1[}\left|\widehat{\psi}^{\prime \prime}\right|^{2} d x: \widehat{\psi} \in \widehat{Y}\right\}=12$ and $\gamma_{0}=2 \sqrt{2 / 3}$.

Step 3. There exists $\nu_{3}>0$ such that

$$
\left.\sum_{j}\left|\zeta_{j}\right|^{(2+a(1+\delta)) /(4-a(1+\delta))} \leq C, \quad \nu \in\right] 0, \nu_{3}[,
$$

where $C$ is a positive constant independent of $\nu$.

Recalling that $\phi$ is even and that on each interval $] \alpha_{j}, \beta_{j}\left[\right.$ the function $u_{j}^{\prime}$ does not change sign, applying Lemma 3.1 we have, for $\nu>0$ small enough (independent of $j$ ),

$$
\begin{aligned}
\int_{] \alpha_{j}, \beta_{j}[} \frac{\phi\left(u_{\nu}^{\prime}\right)}{\phi(1 / \nu)} d x & =\int_{] \alpha_{j}, \beta_{j}[} \frac{\phi\left(\left|w_{j}^{\prime}\right|+\frac{1}{\nu^{\theta}}\right)}{\phi(1 / \nu)} d x \\
& \geq(1-\delta) \int_{] \alpha_{j}, \beta_{j}[}\left(\nu\left|w_{j}^{\prime}\right|+\nu^{1-\theta}\right)^{a(1-\varrho \delta)} d x
\end{aligned}
$$

where

It follows

$$
\int_{] \alpha_{j}, \beta_{j}[} \frac{\phi\left(u_{\nu}^{\prime}\right)}{\phi(1 / \nu)} d x \geq(1-\delta) \int_{] \alpha_{j}, \beta_{j}[}\left(\nu\left|w_{j}^{\prime}\right|\right)^{a(1-\varrho \delta)} d x .
$$

We now claim that

$$
\sum_{j} \int_{] \alpha_{j}, \beta_{j}[}\left(\nu\left|w_{j}^{\prime}\right|\right)^{a(1-\varrho \delta)} d x \geq \sum_{j} \int_{] \alpha_{j}, \beta_{j}[}\left(\nu\left|w_{j}^{\prime}\right|\right)^{a(1+\delta)} d x-C_{0} \delta \nu,
$$

where $C_{0}$ is a positive constant independent of $\nu$. 
Indeed, for all $j \geq 1$, we have

$$
\begin{aligned}
& \int_{] \alpha_{j}, \beta_{j}[}\left(\nu\left|w_{j}^{\prime}\right|\right)^{a(1-\varrho \delta)} d x \\
= & \int_{] \alpha_{j}, \beta_{j}\left\lceil\backslash S_{1 / \nu}\right.}\left(\nu\left|w_{j}^{\prime}\right|\right)^{a(1-\varrho \delta)} d x+\int_{] \alpha_{j}, \beta_{j}\left[\cap S_{1 / \nu}\right.}\left(\nu\left|w_{j}^{\prime}\right|\right)^{a(1-\varrho \delta)} d x \\
\geq & \int_{] \alpha_{j}, \beta_{j}\left\lceil\backslash S_{1 / \nu}\right.}\left(\nu\left|w_{j}^{\prime}\right|\right)^{a(1+\delta)}+\int_{] \alpha_{j}, \beta_{j}\left[\cap S_{1 / \nu}\right.}\left(\nu\left|w_{j}^{\prime}\right|\right)^{a(1-\varrho \delta)} d x \\
= & \int_{] \alpha_{j}, \beta_{j}[}\left(\nu\left|w_{j}^{\prime}\right|\right)^{a(1+\delta)} d x+\int_{] \alpha_{j}, \beta_{j}\left[\cap S_{1 / \nu}\right.}\left[\left(\nu\left|w_{j}^{\prime}\right|\right)^{a(1-\varrho \delta)}-\left(\nu\left|w_{j}^{\prime}\right|\right)^{a(1+\delta)}\right] d x .
\end{aligned}
$$

Now we recall that, from (3.13) we have $\nu\left|w_{j}^{\prime}\right| \leq(1+2 E)$. Moreover, for $x \in S_{1 / \nu}$, we have $\nu\left|w_{j}^{\prime}(x)\right| \geq 1-\nu^{1-\theta} \geq 1 / 2$ for $\nu>0$ small enough. Hence, using (3.12), and observing that the map $\delta \rightarrow \xi^{a(1-\rho(x) \delta)}-\xi^{a(1+\delta)}$ has, for $\xi \in[1 / 2,1+2 E]$, a derivative bounded by a constant $C_{1}$ depending on $E$ but independent of $\nu$, we have

$$
\sum_{j} \int_{] \alpha_{j}, \beta_{j}\left[\cap S_{1 / \nu}\right.}\left|\left(\nu\left|w_{j}^{\prime}\right|\right)^{a(1-\varrho \delta)}-\left(\nu\left|w_{j}^{\prime}\right|\right)^{a(1+\delta)}\right| d x \leq \delta C_{1}\left|S_{1 / \nu}\right| \leq 2 C_{1} E \delta \nu .
$$

Hence the claim follows from (4.24), with $C_{0}:=2 C_{1} E$.

From (4.22) and (4.23) we deduce that

$$
\sum_{j} \int_{] \alpha_{j}, \beta_{j}[} \frac{\phi\left(u_{\nu}^{\prime}\right)}{\nu \phi(1 / \nu)} d x \geq(1-\delta) \sum_{j} \int_{] \alpha_{j}, \beta_{j}[} \frac{1}{\nu}\left(\nu\left|w_{j}^{\prime}\right|\right)^{a(1+\delta)} d x-C_{0} \delta
$$

For all $j$ let $\left.\psi_{j}:\right] 0,1[\rightarrow \mathbb{R}$ be defined by

$$
\left.w_{j}(x)=: \zeta_{j} \psi_{j}\left(\frac{x-\alpha_{j}}{\beta_{j}-\alpha_{j}}\right), \quad x \in\right] \alpha_{j}, \beta_{j}[
$$

Then $\psi_{j}(0)=0, \psi_{j}(1)=1, \psi_{j}^{\prime}(0)=\psi_{j}^{\prime}(1)=0$. Moreover,

$$
\begin{gathered}
\int_{] \alpha_{j}, \beta_{j}[} \frac{1}{\nu}\left(\nu\left|w_{j}^{\prime}\right|\right)^{a(1+\delta)} d x=\left(\frac{\beta_{j}-\alpha_{j}}{\nu}\right)^{1-a(1+\delta)}\left|\zeta_{j}\right|^{a(1+\delta)} \int_{0}^{1}\left|\psi_{j}^{\prime}\right|^{a(1+\delta)} d t \\
=\eta_{j}^{1-a(1+\delta)}\left|\zeta_{j}\right|^{a(1+\delta)} \int_{0}^{1}\left|\psi_{j}^{\prime}\right|^{a(1+\delta)} d t \\
\int_{] \alpha_{j}, \beta_{j}[} \nu^{3}\left|w_{j}^{\prime \prime}\right|^{2} d x=\frac{\nu^{3}}{\left(\beta_{j}-\alpha_{j}\right)^{3}} \zeta_{j}^{2} \int_{0}^{1}\left|\psi_{j}^{\prime \prime}\right|^{2} d t=\frac{\zeta_{j}^{2}}{\eta_{j}^{3}} \int_{0}^{1}\left|\psi_{j}^{\prime \prime}\right|^{2} d t .
\end{gathered}
$$


From the first equality in (4.21), the fact that $\phi$ is nondecreasing, (4.26) and (4.16) it follows

$$
\begin{aligned}
& \sum_{j} F_{\nu}\left(u_{\nu},\right] \alpha_{j}, \beta_{j}[) \geq \sum_{j} F_{\nu}\left(w_{j},\right] \alpha_{j}, \beta_{j}[) \\
& \geq \frac{1}{2}(1-\delta) \sum_{j}\left[\eta_{j}^{1-a(1+\delta)}\left|\zeta_{j}\right|^{a(1+\delta)} \int_{0}^{1}\left|\psi_{j}^{\prime}\right|^{a(1+\delta)} d t+\frac{\zeta_{j}^{2}}{\eta_{j}^{3}} \int_{0}^{1}\left|\psi_{j}^{\prime \prime}\right|^{2} d t\right]-C_{0} \delta \\
& \quad \geq \frac{1}{2}(1-\delta) \sum_{j} \inf \left\{\int_{] 0,1[}\left[\eta^{1-a(1+\delta)}\left|\zeta_{j}\right|^{a(1+\delta)}\left|\widehat{\psi}^{\prime}\right|^{a(1+\delta)}+\frac{\zeta_{j}^{2}}{\eta^{3}}\left|\widehat{\psi}^{\prime \prime}\right|^{2}\right] d x:\right. \\
& \eta>0, \widehat{\psi} \in \widehat{Y}\}-C_{0} \delta \\
& \quad(1-\delta) \sum_{j} \mathrm{e}_{a(1+\delta)}\left(\zeta_{j}\right)-C_{0} \delta=\gamma_{a(1+\delta)}(1-\delta) \sum_{j}\left|\zeta_{j}\right|^{\frac{2+a(1+\delta)}{4-a(1+\delta)}}-C_{0} \delta .
\end{aligned}
$$

Hence step 3 follows.

Step 4. Let $\gamma \in] 0,1\left[, B>0\right.$ and let $\left\{z_{j}\right\}$ be any nonincreasing sequence of nonnegative real numbers such that

$$
\sum_{j=1}^{\infty} z_{j} \geq 1, \quad \sum_{j=1}^{\infty} z_{j}^{\gamma} \leq B .
$$

Then for any $\mu>0$ there exists $N \in \mathbb{N}$ depending on $\gamma, B$ and $\mu$ but independent of $\left\{z_{j}\right\}$, such that

$$
\sum_{j>N} z_{j}<\mu
$$

Given $n \in \mathbb{N}$, we have $n z_{n}^{\gamma} \leq \sum_{j=1}^{n} z_{j}^{\gamma} \leq B$ so that $z_{n} \leq\left(\frac{B}{n}\right)^{1 / \gamma}$. Given $\mu>0$, we select $N$ in such a way that $B^{1 / \gamma} \sum_{j>N} j^{-1 / \gamma} \leq \mu$. We then have $\sum_{j>N} z_{j} \leq$ $B^{1 / \gamma} \sum_{j>N} \frac{1}{j^{1 / \gamma}} \leq \mu$, which concludes the proof of step 4 .

Possibly passing to a further subsequence (still denoted by $\left\{u_{\nu}\right\}$ ) we can assume that, for all $j \in \mathbb{N}$,

$$
\exists \lim _{\nu \rightarrow 0^{+}} \int_{] \alpha_{j}, \beta_{j}[} u_{\nu}^{\prime} d x=: \chi_{j},
$$

with $\sum_{j}\left|\chi_{j}\right|<+\infty($ see $(3.14))$.

Recalling (4.11), we have in particular $\lim _{\nu \rightarrow 0}\left(\beta_{j}-\alpha_{j}\right)=0$. Therefore we can also assume that there exist points $x_{j} \in \bar{I}$ such that

$$
\lim _{\nu \rightarrow 0} \alpha_{j}=\lim _{\nu \rightarrow 0} \beta_{j}=x_{j} .
$$

Since (4.11) implies that $\lim _{\nu \rightarrow 0} \nu^{1-\theta} \eta_{j}=0$ for all $j$, from (4.12) we have

$$
\chi_{j}=\lim _{\nu \rightarrow 0} \zeta_{j}
$$


Step 5. For any $\mu>0$ there exist $N_{\mu}$ dependent on $\mu$ but independent of $\nu$ and a nondecreasing function $\left.\omega_{5}:\right] 0, \min \left\{\nu_{1}, \nu_{2}, \nu_{3}\right\}[\rightarrow] 0,+\infty\left[\right.$, with $\lim _{\nu \rightarrow 0^{+}} \omega_{5}(\nu)=0$, such that

$$
u_{\nu}(x)=u_{\nu}(\alpha)+\sum_{j=1}^{N_{\mu}} \int_{] \alpha_{j}, \beta_{j}[\cap] \alpha, x[} u_{\nu}^{\prime} d t+\mu+\omega_{5}(\nu),
$$

for any $x \in I$ and $\nu \in] 0, \min \left\{\nu_{1}, \nu_{2}, \nu_{3}\right\}[$.

Recalling (4.9), (4.8) and (4.20), we can apply step 4 to $z_{j}:=\left|\zeta_{j}\right|$, to obtain that for any $\mu>0$ there exists $N_{\mu}>0$ depending on $\mu$ but independent of $\nu$ such that

$$
\sum_{j>N_{\mu}}\left|\zeta_{j}\right|<\mu
$$

For any $x \in I$ we can write

$$
u_{\nu}(x)=u_{\nu}(\alpha)+\sum_{j=1}^{N_{\mu}} \int_{] \alpha_{j}, \beta_{j}[\cap] \alpha, x[} u_{\nu}^{\prime} d t+\sum_{j>N_{\mu}} \int_{] \alpha_{j}, \beta_{j}[\cap] \alpha, x[} u_{\nu}^{\prime} d t+\int_{\left.\left(I \backslash S_{\frac{1}{\nu^{\theta}}}\right) \cap\right] \alpha, x[} u_{\nu}^{\prime} d t .
$$

Using (4.13), (4.33) and (4.11) we get

$$
\begin{aligned}
\sum_{j>N_{\mu}}\left|\int_{] \alpha_{j}, \beta_{j}[} u_{\nu}^{\prime} d t\right| & \leq \sum_{j>N_{\mu}}\left(\left|\zeta_{j}\right|+\nu^{1-\theta} \eta_{j}\right) \leq \mu+\nu^{1-\theta} \sum_{j>N_{\mu}} \eta_{j} \\
& \leq \mu+4 E \nu^{(1-\theta)(1-a(1+\delta))}, \quad \nu \leq \nu_{2} .
\end{aligned}
$$

Inserting (4.35) into (4.34) and using (4.4) in step 1, we obtain (4.32) with $\omega_{5}(\nu):=$ $\omega_{1}(\nu)+4 E \nu^{(1-\theta)(1-a(1+\delta))}$, and step 5 is proved.

We are now in a position to conclude the proof. Possibly passing to a subsequence, we can assume that there exists $\lim _{\nu \rightarrow 0} u_{\nu}(\alpha)=: \chi_{0}$. Define

$$
Q:=\left\{x \in I \backslash \bigcup_{j}\left\{x_{j}\right\}: \lim _{\nu \rightarrow 0} u_{\nu}(x)=u(x)\right\} .
$$

Since $\left\{u_{\nu}\right\}$ converges to $u$ almost everywhere in $I$, we have $|I \backslash Q|=0$. Fix now $j \in\left\{1, \ldots, N_{\mu}\right\}$; observe that, if $x \in Q$, then there exists $\tau>0$ such that either $\beta_{j}<x-\tau$ for $\nu$ small enough, or $\alpha_{j}>x+\tau$ for $\nu$ small enough. Hence passing to the limit as $\nu \rightarrow 0^{+}$into (4.32) we deduce

$$
u(x)=\chi_{0}+\sum_{j=1, \ldots, N_{\mu}, x_{j}<x} \chi_{j}+\mu, \quad x \in Q .
$$

Passing to the limit as $\mu \rightarrow 0^{+}$in (4.36) we get

$$
u(x)=\chi_{0}+\sum_{j: x_{j}<x} \chi_{j}, \quad x \in Q .
$$

Since $Q$ is of full measure, (4.37) implies $u \in \mathcal{P}(I)$.

Remark 4.3. The numbers $x_{j}$ appearing in 4.30) do not coincide, in general, with the jump points of the limit function $u$. Indeed, it may happen that some of the limit points $x_{j}$ coincide; see the proof of Theorem 4.4 below for the relations between the representation (4.37) of $u$ and its canonical representation in the form (2.3). 
Theorem 4.4. We have

$$
\Gamma\left(L^{1}(I)\right)-\lim _{\nu \rightarrow 0^{+}} F_{\nu}=\mathcal{F} .
$$

Proof. We first prove that $\mathcal{F} \leq \Gamma\left(L^{1}(I)\right)-\liminf _{\nu} F_{\nu}$. Let $u \in L^{1}(I)$ and let $\left\{u_{\nu}\right\}_{\nu} \subset H^{2}(I)$ be a sequence converging to $u$ in $L^{1}(I)$. We have to prove that

$$
\mathcal{F}(u) \leq \liminf _{\nu \rightarrow 0^{+}} F_{\nu}\left(u_{\nu}\right) .
$$

We can assume that $\liminf _{\nu} F_{\nu}\left(u_{\nu}\right)<+\infty$; otherwise there is nothing to prove. Possibly passing to a subsequence (still denoted by $\left\{u_{\nu}\right\}$ ), we can assume that the $\lim \inf _{\nu} F_{\nu}\left(u_{\nu}\right)$ is a limit and that

$$
E:=\sup _{\nu} F_{\nu}\left(u_{\nu}\right)<+\infty
$$

We follow the notation used in the proof of Theorem 4.1. Thanks to (4.39) and to Theorem 4.1, we have that $u \in \mathcal{P}(I)$. From (4.27) and (4.31) we have, for $\delta>0$ sufficiently small and for a fixed $N \in \mathbb{N}$,

$$
\lim _{\nu \rightarrow 0} F_{\nu}\left(u_{\nu}\right) \geq \liminf _{\nu \rightarrow 0} \sum_{j} F_{\nu}\left(u_{\nu},\right] \alpha_{j}, \beta_{j}[) \geq \gamma_{a(1+\delta)}(1-\delta) \sum_{j=1}^{N}\left|\chi_{j}\right|^{\frac{2+a(1+\delta)}{4-a(1+\delta)}}-C_{0} \delta
$$

Letting first $\delta \rightarrow 0$ and then $N \rightarrow+\infty$ we deduce

$$
\lim _{\nu \rightarrow 0^{+}} F_{\nu}\left(u_{\nu}\right) \geq \gamma_{a} \sum_{j}\left|\chi_{j}\right|^{\frac{2+a}{4-a}} .
$$

Let $j, k \in \mathbb{N}$; write $j \sim k$ if $x_{j}=x_{k}$, and set $[j]:=\left\{k \in \mathbb{N}: k \sim j, x_{k} \notin\{\alpha, \beta\}\right\}$. From (4.41) we have

$$
\lim _{\nu \rightarrow 0^{+}} F_{\nu}\left(u_{\nu}\right) \geq \gamma_{a} \sum_{i} \sum_{h \in\left[j_{i}\right]}\left|\chi_{h}\right|^{\frac{2+a}{4-a}} .
$$

Using the subadditivity of the function $\xi \rightarrow|\xi|^{\frac{2+a}{4-a}}$, we have $\sum_{h \in\left[j_{i}\right]}\left|\chi_{h}\right|^{\frac{2+a}{4-a}} \geq$ $\left|s_{i}\right|^{\frac{2+a}{4-a}}$ for all $i$, where $s_{i}:=\sum_{h \in\left[j_{i}\right]} \chi_{h}$. Hence from (4.42) we get

$$
\lim _{\nu \rightarrow 0^{+}} F_{\nu}\left(u_{\nu}\right) \geq \gamma_{a} \sum_{i}\left|s_{i}\right|^{\frac{2+a}{4-a}} \geq \gamma_{a} \sum_{i: s_{i} \neq 0}\left|s_{i}\right|^{\frac{2+a}{4-a}}=\gamma_{a} \sum_{x \in J_{u}} \mid u\left(x_{+}\right)-u\left(x_{-}\right)^{\frac{2+a}{4-a}} .
$$

Inequality (4.38) is proved.

We now prove that $\mathcal{F} \geq \Gamma\left(L^{1}(I)\right)-\lim \sup _{\nu} F_{\nu}$, i.e., for $u \in L^{1}(I)$ we have to show that there exists a sequence $\left\{u_{\nu}\right\} \subset H^{2}(I)$ converging to $u$ in $L^{1}(I)$ such that

$$
\mathcal{F}(u) \geq \limsup _{\nu \rightarrow 0^{+}} F_{\nu}\left(u_{\nu}\right) .
$$

Obviously, we can assume that $u \in \operatorname{Dom}(\mathcal{F})$. Since $u$ can be approximated in $L^{1}(I)$ by a sequence $\left\{u_{n}\right\}_{n} \subset \mathcal{P}_{\text {fin }}(I)$ of functions such that $\lim _{n} \mathcal{F}\left(u_{n}\right)=\mathcal{F}(u)$, we can assume that $J_{u}$ consists of a finite set of points in $I$. By a standard localization argument, we can also suppose that $J_{u}=\{\bar{x}\}$. Set $\bar{s}:=u\left(\bar{x}_{+}\right)-u\left(\bar{x}_{-}\right)$. With no loss of generality, we can assume $\bar{s}>0$. We will follow [39]. Fix $b>0$ and let $(\eta, \psi) \in] 0,+\infty\left[\times Y_{\eta}(\bar{s})\right.$ be a pair such that

$$
\int_{] 0, \eta[}\left[\left|\psi^{\prime \prime}\right|^{2}+\left|\psi^{\prime}\right|^{a}\right] d x<\mathrm{e}_{a}(\bar{s})+b
$$




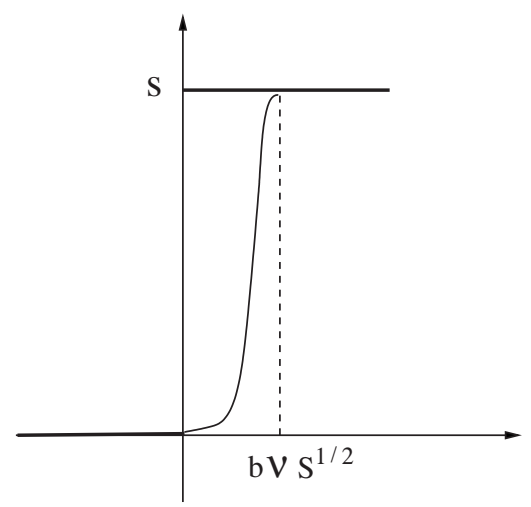

FIguRE 2. Here $a=0$. Given $s>0$, the transition for the optimal sequence takes place in a neighbourhood of size of the order $\nu s^{1 / 2}$ (the constant $b$ equals $\sqrt{6}$ ). The function in the transition layer is a cubic.

Define

$$
u_{\nu}(x):= \begin{cases}u\left(\bar{x}_{-}\right) & x<\bar{x} \\ \psi\left(\frac{x-\bar{x}}{\nu}\right)+u\left(\bar{x}_{-}\right) & x \in[\bar{x}, \bar{x}+\eta \nu[ \\ u\left(\bar{x}_{+}\right) & x \geq \bar{x}+\eta \nu\end{cases}
$$

Then $u_{\nu} \rightarrow u$ in $L^{1}(I)$ as $\nu \rightarrow 0^{+}$and $F_{\nu}\left(u_{\nu},\right] \alpha, \bar{x}[)=F_{\nu}\left(u_{\nu},\right] \bar{x}+\eta \nu, \beta[)=0$. Moreover

$$
F_{\nu}\left(u_{\nu}\right)=F_{\nu}\left(u_{\nu},\right] \bar{x}, \bar{x}+\eta \nu[)=\int_{] 0, \eta[}\left[\left|\psi^{\prime \prime}\right|^{2}+\frac{\phi\left(\psi^{\prime} / \nu\right)}{\phi(1 / \nu)}\right] d x
$$

Since $\psi^{\prime} \in L^{\infty}(] 0, \eta[)$ and $\frac{\phi\left(\psi^{\prime} / \nu\right)}{\phi(1 / \nu)} \leq 2\left\|\psi^{\prime}\right\|_{L^{\infty}(] 0, \eta[)}$ for $\nu>0$ small enough, by the dominated convergence theorem we deduce

$$
\limsup _{\nu \rightarrow 0^{+}} F_{\nu}\left(u_{\nu}\right) \leq \mathrm{e}_{a}\left(u\left(\bar{x}_{+}\right)-u\left(\bar{x}_{-}\right)\right)+b .
$$

Letting $b \rightarrow 0^{+}$, inequality (4.43) follows.

Remark 4.5. Theorems 4.1 and 4.4 hold also with respect to the $L^{p}(I)$-topology, for all $p \in] 1,+\infty[$.

Remark 4.6. Assume that $a=0$. Then the optimal sequence $\left\{u_{\nu}\right\}$ for the construction leading to the $\Gamma$-limsup inequality in Theorem 4.4 can be described as follows. From (4.19) it follows that

$$
\mathrm{e}_{0}(s)=\frac{1}{2} \inf _{\eta>0}\left\{\eta+\int_{] 0, \eta[}\left|\psi_{\mathrm{opt}}^{\prime \prime}\right|^{2} d x\right\}=\frac{1}{2}\left\{\eta_{\mathrm{opt}}+12 s^{2} \eta_{\mathrm{opt}}^{-3}\right\},
$$

where $\psi_{\mathrm{opt}}(s)=-\frac{2 s^{3}}{\eta^{3}}+\frac{3 s^{2}}{\eta^{2}}$ and $\eta_{\mathrm{opt}}^{2}=6 s$. It follows that when $u$ is a two-valued function jumping from 0 to $s$ at the origin, $u_{\nu}$ has the form depicted in Figure 2 , 
4.1. On the convergence of solutions to the fourth order equation. Let $u_{\nu}\left(x, t, \bar{u}_{\nu}\right)$ be the solution of the gradient flow associated with $F_{\nu}$, i.e., the solution to (1.10), (1.11), assuming the validity of (1.12). Concerning boundary conditions on $\partial I \times] 0,+\infty\left[\right.$, different choices can be made; for instance the choices $u_{x}=u_{x x x}=$ 0 , or $u_{x x}=\frac{1}{2 \nu \phi(1 / \nu)} \phi^{\prime}\left(u_{x}\right)-\nu^{3} u_{x x x}=0$ are such that the space integral of $u$ remains constant in time.

Theorem 4.7. Let $\mathcal{T}>0$. There exist a subsequence (still denoted by $\left\{u_{\nu}\right\}$ ) and a function $u: I \times[0, \mathcal{T}[\rightarrow \mathbb{R}$ such that

(i) $\left\{u_{\nu}\right\}$ converges to $u$ in $L^{2}(I \times] 0, \mathcal{T}[)$ as $\nu \rightarrow 0^{+}$.

(ii) $u \in B V(I \times] 0, \mathcal{T}[)$, and $u(\cdot, \tau) \in \mathcal{P}(I)$ for almost every $\tau \in] 0, \mathcal{T}[$.

(iii) $\sup _{\tau \in[0, \mathcal{T}[} \int_{I}|D u(\cdot, \tau)|<+\infty$.

(iv) The map $\tau \rightarrow u(\cdot, \tau)$ has derivative in $L^{2}(I \times] 0, \mathcal{T}[)$.

(v) For each $x \in I$ let $u^{x}(\cdot):=u(x, \cdot)$. Then the atomic part of the measure $D u^{x}$ is zero for almost every $x \in I$.

Proof. Assertions (i), (ii), (iii) follow from (3.14), Theorem 4.1, and

$$
\int_{I \times] 0, \mathcal{T}[}\left|u_{\nu \tau}\right|^{2} d x d \tau=F_{\nu}\left(\bar{u}_{\nu}\right)-F_{\nu}\left(u_{\nu}(\tau)\right) \leq F_{\nu}\left(\bar{u}_{\nu}\right) .
$$

Assertion (iv) follows from the weak lower semicontinuity of the $L^{2}$-norm.

Denote by $\left.J_{u} \subset I \times\right] 0, \mathcal{T}$ [ the jump set of $u$. Since $J_{u}$ is countably $\mathcal{H}^{1}$-rectifiable [5], given $\delta>0$ there is an open set $\left.J_{\delta} \subset I \times\right] 0, T$ [ that covers $J$ and such that $\mathcal{H}^{2}\left(J_{\delta}\right)<\delta$. From [5, Theorem 3.103] we have

$$
\int_{J_{\delta}}\left|D_{\tau} u\right|=\int_{I} \int_{\mathcal{J}_{\delta}^{x}}\left|D u^{x}\right| d x
$$

where $\mathcal{J}_{\delta}^{x}:=\{\tau \in] 0, T\left[:(x, \tau) \in J_{\delta}\right\}$ for any $x \in \mathcal{I}$. From the $L^{1}\left(J_{\delta}\right)$-lower semicontinuity of $\int\left|D_{\tau} u\right|$, (4.45), (4.44) and the Hölder's inequality, we have

$$
\int_{I} \int_{\mathcal{J}_{\delta}^{x}}\left|D u^{x}\right| d x \leq \liminf _{\nu \rightarrow 0^{+}} \int_{J_{\delta}}\left|u_{\nu \tau}\right| d x d \tau \leq \delta^{1 / 2}\left(F_{\nu}\left(\bar{u}_{\nu}\right)\right)^{1 / 2} .
$$

Hence, by Fatou's lemma, $\liminf _{\delta \rightarrow 0^{+}} \int_{\mathcal{J}_{\delta}^{x}}\left|D u^{x}\right|=0$ for almost every $x \in I$. In particular, $\sum_{\tau \in \mathcal{I}^{x}}\left|u^{x}\left(\tau_{+}\right)-u^{x}\left(\tau_{-}\right)\right|=0$ for almost every $x \in I$, where $\mathcal{I}^{x}:=\{\tau \in] 0, \mathcal{T}[$ : $\left.(x, \tau) \in J_{u}\right\}$.

Remark 4.8. Assertion (v) of Theorem 4.7 can be interpreted by saying that the jump points of the limit function $u(\tau)$ do not change their position with $\tau$.

\section{Gradient flow of $\mathcal{F}$ : Weak solutions starting from $\bar{u} \in \mathcal{P}_{\text {fin }}(l)$}

Our aim in this and in the following section is to study the gradient flow in $L^{2}(I)$ of the functional $\mathcal{F}$, in the case where the initial datum $\bar{u}$ has a finite number of jumps. In Section 7 we extend some of the results to the case of $\bar{u}$ with an infinite number of jumps. As we shall see, $\mathcal{F}$ does not admit a well defined gradient vector field. The appropriate generalized definition of gradient flow is the minimizing movement in the sense of De Giorgi in [22] (see [3], 6]). 
Let $\mathcal{G}:] 1,+\infty\left[\times L^{2}(I) \times L^{2}(I) \rightarrow[0,+\infty]\right.$ be defined as

$$
\mathcal{G}(\lambda, v, w):=\mathcal{F}(v)+\frac{\lambda}{2} \int_{I}|v-w|^{2} d x
$$

Definition 5.1. Let $\bar{u} \in L^{2}(I)$. We say that $u:\left[0,+\infty\left[\rightarrow L^{2}(I)\right.\right.$ is a minimizing movement associated with $\mathcal{F}$ and starting from $\bar{u}$ if there exists $w:] 1,+\infty[\times \mathbb{N} \cup$ $\{0\} \rightarrow L^{2}(I)$ such that $w(\lambda, 0)=\bar{u}$ for any $\left.\lambda \in\right] 1,+\infty[$, and

(i) for each $\lambda \in] 1,+\infty[$ and $k \in \mathbb{N} \cup\{0\}$ :

$$
\mathcal{G}(\lambda, w(\lambda, k+1), w(\lambda, k))=\min \left\{\mathcal{G}(\lambda, v, w(\lambda, k)): v \in L^{2}(I)\right\} ;
$$

(ii) $\lim _{\lambda \rightarrow+\infty} w(\lambda,[\lambda \tau])=u(\tau)$ in $L^{2}(I)$ for any $\tau \in[0,+\infty[$.

In (ii) the symbol [.] stands for the integer part. Observe that $u(0)=\bar{u}$.

Remark 5.2. If $\bar{u} \in \operatorname{Dom}(\mathcal{F})$ and if $u$ is a minimizing movement associated with $\mathcal{F}$ starting from $\bar{u}$, then we have $u(\tau) \in \operatorname{Dom}(\mathcal{F})$ for any $\tau \geq 0$. Indeed, $\mathcal{F}(w(\lambda,[\lambda \tau]+$ $1)) \leq \mathcal{F}(w(\lambda,[\lambda \tau])) \leq \mathcal{F}(\bar{u})$; hence using the $L^{2}$-lower semicontinuity of $\mathcal{F}$ we get $\mathcal{F}(u(\tau)) \leq \liminf _{\lambda \rightarrow+\infty} \mathcal{F}(w(\lambda,[\lambda \tau])) \leq \mathcal{F}(\bar{u})$.

Proposition 5.3. Let $w \in L^{2}(I)$ and $\lambda>1$. Then there exists a function which solves the problem

$$
\min \left\{\mathcal{G}(\lambda, v, w): v \in L^{2}(I)\right\} .
$$

Any solution $v$ of (5.3) belongs to $\operatorname{Dom}(\mathcal{F})$, and if $w \in L^{\infty}(I)$, then $\|v\|_{\infty} \leq\|w\|_{\infty}$.

Proof. As a consequence of (4.1) and (4.4), $\mathcal{G}(\lambda, \cdot, w)$ is $L^{2}(I)$-coercive and $L^{2}(I)$ lower semicontinuous, and the existence assertion follows. If $w \in L^{\infty}(I)$ and $\|v\|_{\infty}>\|w\|_{\infty}$, then the function $\hat{v}:=\left(-\|w\|_{\infty}\right) \vee v \wedge\|w\|_{\infty}$ is such that $\mathcal{G}(\lambda, \hat{v}, w)<$ $\mathcal{G}(\lambda, v, w)$.

The main result of this section is the following theorem.

Theorem 5.4. Let $\bar{u} \in \mathcal{P}_{\text {fin }}(I), \bar{u}=\bar{s}_{0}+\sum_{i=1}^{N} \bar{s}_{i} 1_{\left[\bar{x}_{i}, \beta[\right.}$. Then there exists a unique minimizing movement $u$ starting from $\bar{u}$. Moreover $u:\left[0,+\infty\left[\rightarrow \mathcal{P}_{\text {fin }}(I) \subset L^{2}(I)\right.\right.$ is $\frac{1}{2}$-Hölder continuous, and

a) there exist $M \in \mathbb{N}, M \leq N$, and times $\tau_{1}, \ldots, \tau_{M}$, with $0=: \tau_{0}<\tau_{1}<$ $\cdots<\tau_{M}<+\infty$, with the following properties:

(a1) the set-valued function $\tau \in\left[0,+\infty\left[\rightarrow J_{u(\tau)} \subset I\right.\right.$ is constant on each interval $\left[\tau_{i}, \tau_{i+1}[\right.$ for any $i=0, \ldots, M-1$;

(a2) $\mathcal{H}^{0}\left(J_{u\left(\tau_{i_{+}}\right)}\right)<\mathcal{H}^{0}\left(J_{u\left(\tau_{i_{-}}\right)}\right)$for any $i=1, \ldots, M ; J_{u(\tau)}=\emptyset$ for all $\tau \in\left[\tau_{M},+\infty[\right.$

(a3) $\int_{I} u(\tau) d x=\int_{I} \bar{u} d x$ for any $\tau \in[0,+\infty[$.

b) For $i=0, \ldots, M-1$, writing $u$ in $\left[\tau_{i}, \tau_{i+1}[\right.$ as

$$
u(\tau)=s_{0}(\tau)+\sum_{j=1}^{N_{i}} s_{j}(\tau) 1_{\left[x_{j}(\tau), \beta[\right.},
$$


where $N_{i}:=\mathcal{H}^{0}\left(J_{u\left(\tau_{i_{+}}\right)}\right)$and $N_{0}=N$, we have that the evolution of $u$ in $\left[\tau_{i}, \tau_{i+1}\right.$ [ is determined by the following system of ordinary differential equations: if $N_{i}>2$,

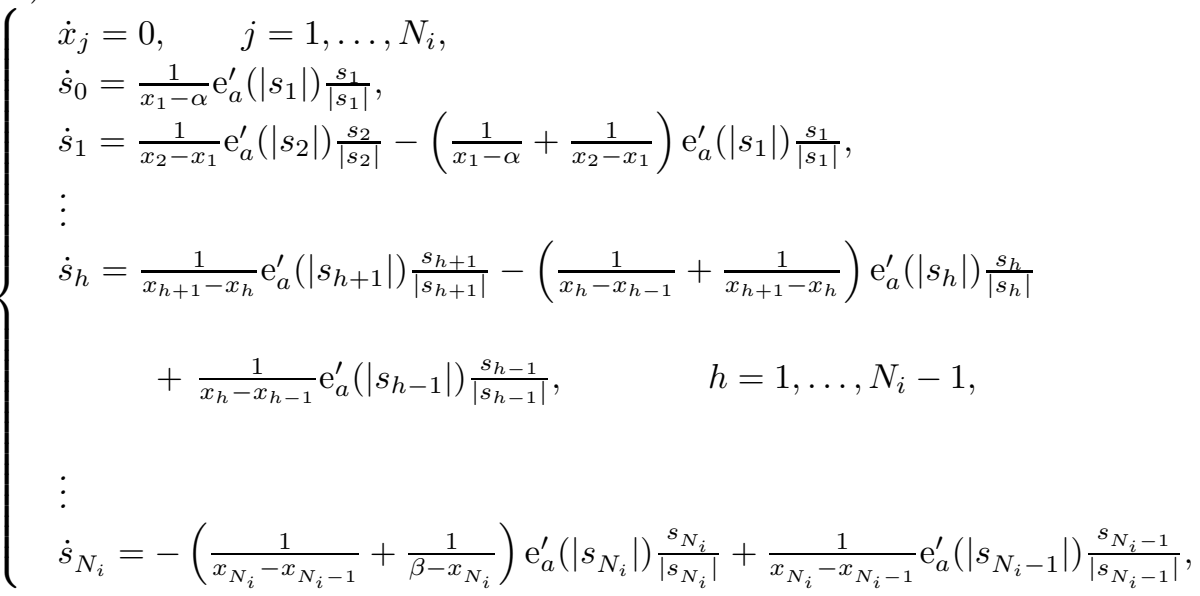

$$
\begin{aligned}
& \text { if } N_{i}=2, \quad\left\{\begin{array}{l}
\dot{x}_{1}=\dot{x}_{2}=0, \\
\dot{s}_{0}=\frac{1}{x_{1}-\alpha} \mathrm{e}_{a}^{\prime}\left(\left|s_{1}\right|\right) \frac{s_{1}}{\left|s_{1}\right|}, \\
\dot{s}_{1}=\frac{1}{x_{2}-x_{1}} \mathrm{e}_{a}^{\prime}\left(\left|s_{2}\right|\right) \frac{s_{2}}{\left|s_{2}\right|}-\left(\frac{1}{x_{1}-\alpha}+\frac{1}{x_{2}-x_{1}}\right) \mathrm{e}_{a}^{\prime}\left(\left|s_{1}\right|\right) \frac{s_{1}}{\left|s_{1}\right|}, \\
\dot{s}_{2}=-\left(\frac{1}{x_{2}-x_{1}}+\frac{1}{\beta-x_{2}}\right) \mathrm{e}_{a}^{\prime}\left(\left|s_{2}\right|\right) \frac{s_{2}}{\left|s_{2}\right|}+\frac{1}{x_{2}-x_{1}} \mathrm{e}_{a}^{\prime}\left(\left|s_{1}\right|\right) \frac{s_{1}}{\left|s_{1}\right|} .
\end{array}\right.
\end{aligned}
$$

We divide the proof of Theorem 5.4 into several parts.

Definition 5.5. For each $w \in \mathcal{P}_{\text {fin }}(I), w=\sigma_{0}+\sum_{j=1}^{\mathcal{H}^{0}\left(J_{w}\right)} \sigma_{j} 1_{\left[x_{j}, \beta[\right.}$, we set

$$
\begin{gathered}
l_{w}:=\min _{x, y \in J_{w}, x \neq y}|x-y|, \\
h_{w}=\min \left\{\left|\sigma_{j}\right|: x_{j} \in J_{w}\right\} .
\end{gathered}
$$

We begin with the following proposition.

Proposition 5.6. The following properties hold.

(i) Let $w \in L^{2}(I)$ and let $v$ be a solution of (5.3). Then $\mathcal{H}^{0}\left(J_{v}\right)<+\infty$.

(ii) Let $w \in \operatorname{Dom}(\mathcal{F})$. Then there exists a solution $\omega$ of $(5.3)$ with $J_{\omega} \subseteq \overline{J_{w}}$.

(iii) Let $w \in \mathcal{P}_{\text {fin }}(I)$. Then any solution $v$ of (5.3) has the property that $\mathcal{H}^{0}\left(J_{v}\right) \leq \mathcal{H}^{0}\left(J_{w}\right)$. Moreover,

$$
|v-w| \text { is constant on each connected component of } I \backslash J_{w},
$$




$$
l_{v} \geq l_{w}
$$

and

$$
\mathcal{H}^{0}\left(J_{v}\right)=\mathcal{H}^{0}\left(J_{w}\right) \Longleftrightarrow J_{v}=J_{w} .
$$

(iv) Let $w \in \mathcal{P}_{\text {fin }}(I)$ and let $v$ be a solution of (5.3). Then there exists a solution $\widehat{v} \in \mathcal{P}_{\text {fin }}(I)$ of (5.3) such that $J_{\widehat{v}} \subseteq J_{w}$ and $\widehat{v}(I)=v(I)$. Therefore $\mathcal{F}(\widehat{v})=$ $\mathcal{F}(v)$ and $\int_{I}|\widehat{v}-w|^{2} d x=\int_{I}|v-w|^{2} d x$.

(v) Let $w \in \mathcal{P}_{\text {fin }}(I)$. If $\lambda>1$ is sufficiently large, then (5.3) admits a unique solution $v$ and $J_{v}=J_{w}$.

Proof. Let $w \in L^{2}(I)$ and let $v \in \operatorname{Dom}(\mathcal{F})$ be a solution of (5.3). We argue by contradiction. Assume $v \notin \mathcal{P}_{\text {fin }}(I), v=s_{0}+\sum_{i \in \mathbb{N}} s_{i} 1_{\left[x_{i}, \beta[\right.}, \sum_{i \in \mathbb{N}}\left|s_{i}\right|^{r}<+\infty$, $s_{i} \neq 0$ for all $i \in \mathbb{N}$, with $x_{i} \neq x_{k}$ for $i \neq k$, and where $r:=\frac{2+a}{4-a}$. For $m \in \mathbb{N}$ let

$$
v_{m}:=s_{0}+\sum_{i=1}^{m} s_{i} 1_{\left[x_{i}, \beta[\right.}, \quad R_{m}:=\gamma_{a} \sum_{i=m+1}^{+\infty}\left|s_{i}\right|^{r} .
$$

Since $\mathcal{F}(v)=\mathcal{F}\left(v_{m}\right)+R_{m}$, we have

$$
\mathcal{G}(\lambda, v, w)-\mathcal{G}\left(\lambda, v_{m}, w\right)=R_{m}+\frac{\lambda}{2}\left(\|v-w\|_{2}^{2}-\left\|v_{m}-w\right\|_{2}^{2}\right) .
$$

Moreover $\mathcal{G}(\lambda, v, w) \leq \mathcal{G}(\lambda, 0, w)$ implies $\|v-w\|_{2} \leq\|w\|_{2}$. Using the triangular inequality we have

$$
\begin{aligned}
\left\|v_{m}-w\right\|_{2}^{2}-\|v-w\|_{2}^{2} & \leq\left(\|v-w\|_{2}+\left\|v_{m}-w\right\|_{2}\right)\left\|v-v_{m}\right\|_{2} \\
& \leq\left(2\|v-w\|_{2}+\left\|v-v_{m}\right\|_{2}\right)\left\|v-v_{m}\right\|_{2} \\
& \leq\left(2\|w\|_{2}+\left\|v-v_{m}\right\|_{2}\right)\left\|v-v_{m}\right\|_{2} .
\end{aligned}
$$

Observe now that

$$
\frac{1}{|I|^{1 / 2}}\left\|v-v_{m}\right\|_{2} \leq\left\|v-v_{m}\right\|_{\infty} \leq \sum_{i=m+1}^{+\infty}\left|s_{i}\right| \leq\left(\frac{R_{m}}{\gamma_{a}}\right)^{1 / r},
$$

where in the last inequality we have used (2.6). From (5.13) and (5.14) we deduce

$$
\left\|v_{m}-w\right\|_{2}^{2}-\|v-w\|_{2}^{2} \leq\left(2\|w\|_{2}+|I|^{1 / 2}\left(\frac{R_{m}}{\gamma_{a}}\right)^{1 / r}\right)|I|^{1 / 2}\left(\frac{R_{m}}{\gamma_{a}}\right)^{1 / r} .
$$

Therefore from (5.12),

$$
\begin{aligned}
& \mathcal{G}(\lambda, v, w)-\mathcal{G}\left(\lambda, v_{m}, w\right) \\
& \quad \geq R_{m}\left(1-\frac{\lambda}{2}\left(2\|w\|_{2}+|I|^{1 / 2}\left(\frac{R_{m}}{\gamma_{a}}\right)^{1 / r}\right) \frac{|I|^{1 / 2}}{\gamma_{a}}\left(\frac{R_{m}}{\gamma_{a}}\right)^{\frac{1}{r}-1}\right) .
\end{aligned}
$$

Since $\lim _{m \rightarrow+\infty} R_{m}=0, R_{m}>0$, and $1 / r>1$, it follows that the left hand side of (5.15) is positive for $m$ large enough. This contradicts the minimality of $v$, and proves that $v$ has a finite number of jumps.

Assume now that $w \in \operatorname{Dom}(\mathcal{F})$.

If $\mathcal{H}^{0}\left(J_{w}\right)$ is infinite, let $\left\{J^{m}\right\}$ be a sequence of sets such that $\mathcal{H}^{0}\left(J^{m}\right)=m$, $J^{m} \subset J^{m+1}$ and $\bigcup_{m} J^{m}=J_{w}$. Write $J^{m}=\left\{\bar{x}_{j}^{m}\right\}$, where $\alpha<\bar{x}_{1}^{m}<\bar{x}_{2}^{m}<\cdots<$ 
$\bar{x}_{m}^{m}<\beta$. Define $w_{m}:=w\left(\alpha_{+}\right)+\sum_{j}\left(w\left(\bar{x}_{j+}^{m}\right)-w\left(\bar{x}_{j_{-}}^{m}\right)\right) 1_{\left[\bar{x}_{j}^{m}, \beta[\right.}$. Let $\left.I_{1}^{m}:=\right] \alpha, \bar{x}_{1}^{m}[$, $I_{j}^{m}:=\left[\bar{x}_{j-1}^{m}, \bar{x}_{j}^{m}\left[\right.\right.$ for $j \in\{2, \ldots, m\}$, and $I_{m+1}^{m}:=\left[\bar{x}_{m}^{m}, \beta[\right.$.

If $\mathcal{H}^{0}\left(J_{w}\right)$ is finite, let $w_{m}:=w$, write $J_{w}=\left\{\bar{x}_{1}, \ldots, \bar{x}_{\mathcal{H}^{0}\left(J_{w}\right)}\right\}$, and let $I_{1}:=$ ]$\alpha, \bar{x}_{1}\left[, I_{j}:=\left[\bar{x}_{j-1}, \bar{x}_{j}\left[\right.\right.\right.$ for $j \in\left\{2, \ldots, \mathcal{H}^{0}\left(J_{w}\right)\right\}$, and $I_{\mathcal{H}^{0}\left(J_{w}\right)+1}:=\left[\bar{x}_{\mathcal{H}^{0}\left(J_{w}\right)}, \beta[\right.$ (in this case we can assume $m=\mathcal{H}^{0}\left(J_{w}\right)$, and we drop the superscript $m$ ).

Let $v \in \operatorname{Dom}(\mathcal{F})$ be a solution of (5.3). By (i) it follows that $v \in \mathcal{P}_{\text {fin }}(I)$. Write $v=s_{0}+\sum_{i=1}^{\mathcal{H}^{0}\left(J_{v}\right)} s_{i} 1_{\left[x_{i}, \beta[\right.}$. To prove (ii) it suffices to construct a function $\omega \in \mathcal{P}_{\text {fin }}(I)$ with $\mathcal{G}(\lambda, \omega, w) \leq \mathcal{G}(\lambda, v, w)$ and $J_{\omega} \subseteq \overline{J_{w}}$. For all $j \in\{1, \ldots, m+1\}$ let $z_{j}^{m} \in I_{j}^{m}$ be such that

$$
\left|v\left(z_{j}^{m}\right)-w_{m}\left(z_{j}^{m}\right)\right|=\min _{x \in I_{j}^{m}}\left|v(x)-w_{m}(x)\right|,
$$

and let $v_{m}^{*} \in \mathcal{P}_{\text {fin }}(I)$ be defined as $v_{m}^{*}(x):=v\left(z_{j}^{m}\right)$ for $x \in I_{j}^{m}$. Then

$$
J_{v_{m}^{*}} \subseteq J_{w}, \quad v_{m}^{*}(I) \subseteq v(I), \quad \mathcal{H}^{0}\left(J_{v_{m}^{*}}\right) \leq \mathcal{H}^{0}\left(J_{v}\right) \quad \forall m \in \mathbb{N},
$$

and $\mathcal{F}\left(v_{m}^{*}\right)=\gamma_{a} \sum_{j=1}^{m}\left|v\left(z_{j+1}^{m}\right)-v\left(z_{j}^{m}\right)\right|^{r}$. Moreover, for any $j \in\{1, \ldots, m+1\}$,

$$
\begin{aligned}
\int_{I_{j}^{m}}\left(v-w_{m}\right)^{2} d x & \geq\left(\min _{x \in I_{j}^{m}}\left|v(x)-w_{m}(x)\right|\right)^{2}\left|I_{j}^{m}\right|=\int_{I_{j}^{m}}\left(v\left(z_{j}^{m}\right)-w_{m}\left(z_{j}^{m}\right)\right)^{2} d x \\
& =\int_{I_{j}^{m}}\left(v_{m}^{*}(x)-w_{m}\left(z_{j}^{m}\right)\right)^{2} d x=\int_{I_{j}^{m}}\left(v_{m}^{*}-w_{m}\right)^{2} d x ;
\end{aligned}
$$

hence

$$
\left\|v_{m}^{*}-w_{m}\right\|_{2} \leq\left\|v-w_{m}\right\|_{2} .
$$

Finally, $v\left(z_{j+1}^{m}\right)-v\left(z_{j}^{m}\right)=\sum_{i \in\{1, \ldots, N\}: z_{j}^{m}<x_{i} \leq z_{j+1}^{m}} s_{i}$. Hence, using the subadditivity of the function $s \rightarrow|s|^{r}$, we have

$$
\begin{aligned}
\mathcal{F}\left(v_{m}^{*}\right) & =\gamma_{a} \sum_{j=1}^{m}\left|v\left(z_{j+1}^{m}\right)-v\left(z_{j}^{m}\right)\right|^{r} \\
& =\gamma_{a} \sum_{j=1}^{m}\left|\sum_{i \in\{1, \ldots, N\}: z_{j}^{m}<x_{i} \leq z_{j+1}^{m}} s_{i}\right|^{r} \leq \gamma_{a} \sum_{i=1}^{\mathcal{H}^{0}\left(J_{v}\right)}\left|s_{i}\right|^{r}=\mathcal{F}(v) .
\end{aligned}
$$

From (5.18) and (5.19) we deduce

$$
\mathcal{G}\left(\lambda, v_{m}^{*}, w_{m}\right) \leq \mathcal{G}\left(\lambda, v, w_{m}\right), \quad m \in \mathbb{N} .
$$

From (5.19), (5.17) and the coercivity of $\mathcal{F}$, there exist a subsequence $\left\{m_{k}\right\}$ and a function $\omega \in \operatorname{Dom}(\mathcal{F})$ such that $\left\{v_{m_{k}}^{*}\right\}$ converges to $\omega$ in $L^{2}(I)$ as $k \rightarrow+\infty$. Observe that (5.17) implies that the number of jumps of $v_{m_{k}}^{*}$ is uniformly bounded with respect to $k$. Hence there exists $l \in \mathbb{N}, l \leq \mathcal{H}^{0}\left(J_{v}\right)$ such that $J_{v_{m_{k}}^{*}}=\left\{y_{1}^{k}, \ldots, y_{l}^{k}\right\}$ for all $k$. Therefore, possibly extracting a further subsequence, we can suppose that $\left\{y_{r}^{k}\right\}$ converges, as $k \rightarrow+\infty$, to a point $y_{r} \in \bar{I}$ for any $r \in\{1, \ldots, l\}$; moreover, by the inclusion in (5.17), it follows that $y_{r} \in \overline{J_{w}}$. 
Since $\left\|v_{m}^{*}-w\right\|_{2} \leq\left\|v_{m}^{*}-w_{m}\right\|_{2}+\left\|w_{m}-w\right\|_{2}$, from (5.20), the lower semicontinuity of $\mathcal{F}$ and the $L^{2}(I)$-convergence of $\left\{w_{m}\right\}$ to $w$, we deduce

$$
\mathcal{G}(\lambda, \omega, w) \leq \mathcal{G}(\lambda, v, w),
$$

so that $\omega$ minimizes $\mathcal{G}(\lambda, \cdot, w)$. Since $J_{\omega} \subseteq\left\{y_{1}, \ldots, y_{r}\right\}$, assertion (ii) follows.

To prove (iii), let $J_{w}=\left\{\bar{x}_{1}, \ldots, \bar{x}_{m}\right\}$ with $\alpha<\bar{x}_{1}<\cdots<\bar{x}_{m}<\beta$, and $J_{v}=$ $\left\{x_{1}, \ldots, x_{\mathcal{H}^{0}\left(J_{v}\right)}\right\}$. We argue by contradiction. Assume that $\mathcal{H}^{0}\left(J_{v}\right)>m$. As in the proof of (ii), we can construct points $z_{1}, \ldots, z_{m}$ satisfying (when $z_{j}$ is substituted for $z_{j}^{m}$ ) formula (5.16), and a function $v^{*}$ which satisfies (when substituted for $v_{m}^{*}$ ) formulae (5.17), (5.18) and (5.19). Then for at least one $j$, the set $\{i \in$ $\left.\left\{1, \ldots, \mathcal{H}^{0}\left(J_{v}\right)\right\}: z_{j}<x_{i} \leq z_{j+1}\right\}$ in (5.19) has cardinality strictly larger than one; hence $\mathcal{H}^{0}\left(J_{v^{*}}\right)<\mathcal{H}^{0}\left(J_{v}\right)$. Then the strict subadditivity of the function $s \rightarrow|s|^{r}$ implies that the last inequality in (5.19) is strict, and therefore $\mathcal{F}\left(v^{*}\right)<\mathcal{F}(v)$, and in turn $\mathcal{G}\left(\lambda, v^{*}, w\right)<\mathcal{G}(\lambda, v, w)$, contradicting the minimality of $v$. It follows that $\mathcal{H}^{0}\left(J_{v}\right) \leq \mathcal{H}^{0}\left(J_{w}\right)$.

Let us prove (5.9), (5.10) and the final assertion of (iii). Assume that (5.9) is false. Let $x_{0} \in I_{j}$ be such that $\left|v\left(x_{0}\right)-w\left(x_{0}\right)\right|>\min _{x \in I_{j}}|v(x)-w(x)|$. Then the function $v^{*}$ defined in the proof of (ii) satisfies $\mathcal{F}\left(v^{*}\right) \leq \mathcal{F}(v)$, and $\int_{I}\left|v^{*}-w\right|^{2} d x<$ $\int_{I}|v-w|^{2} d x$, contradicting the minimality of $v$.

We then notice that $v$ has at most one jump point $\left.\xi_{j} \in\right] \bar{x}_{j-1}, \bar{x}_{j}[$ for any $j \in$ $\{1, \ldots, m+1\}$. Indeed, define $\widetilde{v}:=v\left(\bar{x}_{j-1}\right)$ on $\left[\bar{x}_{j-1}, \frac{\bar{x}_{j-1}+\bar{x}_{j}}{2}\left[, \widetilde{v}:=v\left(\bar{x}_{j_{-}}\right)\right.\right.$on $\left[\frac{\bar{x}_{j-1}+\bar{x}_{j}}{2}, \bar{x}_{j}\left[\right.\right.$, and $\widetilde{v}:=v$ on $I \backslash I_{j}$. Then $\int_{I}|\widetilde{v}-w|^{2} d x=\int_{I}|v-w|^{2} d x$ by the previous observation, and $\mathcal{F}(\widetilde{v}) \leq \mathcal{F}(v)$, with the strict inequality whenever the number of jump points of $v$ in $] \bar{x}_{j-1}, \bar{x}_{j}$ [ is larger than or equal to two. Let us prove that $v$ is constant on $I_{1}$ and on $I_{m+1}$. Indeed, if $v$ has a jump point $\left.\xi_{1} \in\right] \alpha, \bar{x}_{1}[$, then the function $\widetilde{v}$ defined as $\widetilde{v}:=v\left(\bar{x}_{1-}\right)$ on $] \alpha, \bar{x}_{1}[$ and $\widetilde{v}:=v$ on $I \backslash] \alpha, \bar{x}_{1}[$ has $\int_{I}|\widetilde{v}-w|^{2} d x=\int_{I}|v-w|^{2} d x$ and $\mathcal{F}(\widetilde{v})<\mathcal{F}(v)$. The same arguments apply to the interval $] \bar{x}_{m}, \beta[$.

Furthermore, we observe that if $v$ has a jump point $\left.\xi_{j} \in\right] \bar{x}_{j-1}, \bar{x}_{j}$ [ for some $j \in\{2, \ldots, m\}$, then $v$ is constant on $\left[\bar{x}_{j-2}, \xi_{j}\left[\right.\right.$ and on $\left[\xi_{j}, \bar{x}_{j+1}[\right.$. We only discuss the interval $\left[\bar{x}_{j-2}, \xi_{j}\right.$ [ since the analysis of the other interval is similar. The function $\widetilde{v}$ defined as $\widetilde{v}:=v\left(\bar{x}_{j-2}\right)$ on $\left[\bar{x}_{j-2}, \bar{x}_{j-1}\left[, \widetilde{v}:=v\left(\bar{x}_{j_{-}}\right)\right.\right.$on $\left[\bar{x}_{j-1}, \bar{x}_{j}[\right.$ and $\widetilde{v}:=v$ on $\left.I \backslash] \bar{x}_{j-2}, \bar{x}_{j}\right]$ satisfies $\int_{I}|\widetilde{v}-w|^{2} d x=\int_{I}|v-w|^{2} d x$ and $\mathcal{F}(\widetilde{v}) \leq \mathcal{F}(v)$, with the strict inequality if $v$ has a jump point in $] \bar{x}_{j-2}, \bar{x}_{j-1}[$. This proves (5.10) and concludes the proof of (iii).

To prove (iv), it is sufficient to define $\widehat{v}:=v\left(\bar{x}_{j-1}\right)$ on $I_{j}$ and to use (iii).

In order to prove (v), let $v \in \mathcal{P}_{\text {fin }}(I)$ be a solution of (5.3). From Lemma 5.7 below it follows that, if $\lambda>1$ is sufficiently large, then $\mathcal{H}^{0}\left(J_{v}\right) \geq \mathcal{H}^{0}\left(J_{w}\right)$. Hence, by (iii), we have $\mathcal{H}^{0}\left(J_{v}\right)=\mathcal{H}^{0}\left(J_{w}\right)$. The functional $\mathcal{F}$, restricted to the finitedimensional subset $X$ of functions in $\mathcal{P}_{\text {fin }}(I)$ having exactly $\mathcal{H}^{0}\left(J_{w}\right)$ jumps, is a $\mathcal{C}^{\infty}$ function. Therefore, for $\lambda>1$ sufficiently large, $\mathcal{G}(\lambda, \cdot, w)$ becomes strictly convex on $X$, hence has a unique minimizer $v$. The equality $J_{v}=J_{w}$ follows from (iii). 
The next lemma states, in particular, that the map $w \in \mathcal{P}_{\text {fin }}(I) \rightarrow \mathcal{H}^{0}\left(J_{w}\right)$ is $L^{2}(I)$-lower semicontinuous.

Lemma 5.7. Let $w \in \mathcal{P}_{\text {fin }}(I), w=\sigma_{0}+\sum_{h=1}^{\mathcal{H}^{0}\left(J_{w}\right)} \sigma_{h} 1_{\left[y_{h}, \beta[\right.}$. Then there exists $\delta>0$ such that, if $v \in \mathcal{P}_{\text {fin }}(I)$ satisfies $\|v-w\|_{2}<\delta$, then

$$
\mathcal{H}^{0}\left(J_{v}\right) \geq \mathcal{H}^{0}\left(J_{w}\right)
$$

and

$$
\begin{aligned}
\left.\forall h \in\left\{0, \ldots, \mathcal{H}^{0}\left(J_{w}\right)\right\} \quad \exists \xi_{h} \in\right] y_{h}, y_{h+1}[:] \xi_{h}, \xi_{h+1}[ & \cap J_{w}=\left\{y_{h+1}\right\}, \\
] & \xi_{h}, \xi_{h+1}\left[\cap J_{v} \neq \emptyset,\right.
\end{aligned}
$$

where $y_{0}:=\alpha, y_{\mathcal{H}^{0}\left(J_{w}\right)+1}:=\beta$.

Proof. Let $v=s_{0}+\sum_{j=1}^{\mathcal{H}^{0}\left(J_{v}\right)} s_{j} 1_{\left[x_{j}, \beta[\right.}$. For all $h=0, \ldots, \mathcal{H}^{0}\left(J_{w}\right)$, let $d_{h}:=$ $\min _{x \in\left[y_{h}, y_{h+1}[\right.}\left|w\left(y_{h}\right)-v(x)\right|$. Then $\sum_{h=0}^{\mathcal{H}^{0}\left(J_{w}\right)} d_{h}^{2}\left(y_{h+1}-y_{h}\right) \leq\|v-w\|_{2}^{2}$; hence $d_{h} \leq$ $l_{w}^{-1 / 2}\|v-w\|_{2}$. It follows that for each $h=0, \ldots, \mathcal{H}^{0}\left(J_{w}\right)$ there exists $\left.\xi_{h} \in\right] y_{h}, y_{h+1}[$ such that $d_{h}=\left|w\left(y_{h}\right)-v\left(\xi_{h}\right)\right| \leq l_{w}^{-1 / 2}\|v-w\|_{2}$. Since $\sigma_{h}=w\left(y_{h+1}\right)-w\left(y_{h}\right)$ we have

$\left|v\left(\xi_{h+1}\right)-v\left(\xi_{h}\right)-\sigma_{h}\right| \leq\left|v\left(\xi_{h+1}\right)-w\left(y_{h+1}\right)\right|+\left|v\left(\xi_{h}\right)-w\left(y_{h}\right)\right| \leq 2 l_{w}^{-1 / 2}\|v-w\|_{2}$.

If we take $\delta<\frac{l_{w}^{1 / 2} h_{w}}{2}$, we deduce $\left|v\left(\xi_{h+1}\right)-v\left(\xi_{h}\right)-\sigma_{h}\right|<h_{w}$, and hence there must be a jump point of $v$ between $\xi_{h}$ and $\xi_{h+1}$.

Remark 5.8.

(1) Proposition [5.6 (i) can be considered as a regularity result for minimizers of (5.3): if $w$ is an arbitrary function in $L^{2}(I)$, solutions to (5.3) have a finite number of jumps.

(2) Let $w \in L^{2}(I)$ and let $v=s_{0}+\sum_{i} s_{i} 1_{\left[x_{i}, \beta[\right.}$ be a solution of (5.3). Then

$$
\mathcal{H}^{0}\left(J_{v}\right) \leq C \lambda^{\frac{2 r}{1-r}}\|w\|_{2}^{\frac{4-2 r}{1-r}}
$$

where

$$
C:=\frac{|I|^{\frac{r}{1-r}}}{\gamma_{a}^{\frac{4 a}{1-a}} 2^{\frac{2-r}{1-r}}} .
$$

Indeed, let us order the $s_{i}$ in such a way that $\left|s_{1}\right| \geq\left|s_{2}\right| \geq \cdots \geq\left|s_{N}\right|$. From the minimality of $v$ it follows $\mathcal{G}(\lambda, v, w) \leq \mathcal{G}\left(\lambda, v_{N-1}, w\right)$, where $v_{N-1}$ is defined in (5.11) with $m:=N-1$. Inserting this inequality into (5.15) and setting $\eta_{N-1}:=\gamma_{a}\left|s_{N}\right|^{r}$ yields

$$
\left(2\|w\|_{2}+|I|^{1 / 2}\left(\frac{\eta_{N-1}}{\gamma_{a}}\right)^{1 / r}\right) \frac{|I|^{1 / 2}}{\gamma_{a}}\left(\frac{\eta_{N-1}}{\gamma_{a}}\right)^{\frac{1}{r}-1} \geq \frac{2}{\lambda} .
$$

Hence, observing that $\mathcal{F}(v) \leq \frac{\lambda}{2}\|w\|_{2}^{2}$, we have

$$
\eta_{N-1} \geq\left(\frac{2}{\lambda} \frac{1}{2\|w\|_{2}+|I|^{1 / 2}\left(\frac{\lambda}{2}\right)^{1 / r}\|w\|_{2}^{2 / r}} \frac{\gamma_{a}^{2 / r}}{|I|^{1 / 2}}\right)^{\frac{1}{r}-1}
$$

Since $N \eta_{N-1} \leq \mathcal{F}(v)$, using (5.24) we deduce (5.23). 
On the basis of Proposition [5.3, given $\bar{u} \in \mathcal{P}_{\text {fin }}(I)$ and $\lambda>1$, there exists a sequence $\{w(\lambda, k)\}_{k}, w(\lambda, 0)=\bar{u}$, with the property that, for each $k \in \mathbb{N} \cup\{0\}$, $w(\lambda, k+1)$ is a solution of (5.2). Examples show that (5.2) may have more than one solution (see Example 2 in Section 5.2 below). It follows that, in general, the sequence $\{w(\lambda, k)\}_{k}$ is not uniquely determined. Therefore, to prove Theorem 5.4 we need to show that, independently of the particular sequence $\{w(\lambda, k)\}_{k}$ considered

$$
\lim _{\lambda \rightarrow+\infty}\|w(\lambda,[\lambda \tau])-u(\tau)\|_{2}=0 \quad \forall \tau \in[0,+\infty[,
$$

where $u(\tau)$ is determined by (5.4)-(5.6) .

Definition 5.9. We define $\Sigma_{1}$ as the finite dimensional subset of $\mathcal{P}_{\text {fin }}(I)$ of the functions $w$ such that

$$
\left\{\begin{array}{l}
w=\sigma_{0}+\sum_{j=1}^{N} \sigma_{j} 1_{\left[\bar{x}_{j}, \beta[\right.}, \\
\operatorname{sign}\left(\sigma_{j}\right)=\operatorname{sign}\left(\bar{s}_{j}\right), \quad 1 \leq j \leq N
\end{array}\right.
$$

(recall that $\left.\bar{u}=\bar{s}_{0}+\sum_{j=1}^{N} \bar{s}_{j} 1_{\left[\bar{x}_{j}, \beta[\right.}\right)$.

Lemma 5.10. There exist $\bar{\lambda}>1$ and $\bar{\tau}>0$ such that

$$
w(\lambda, k) \in \Sigma_{1} \quad \forall \lambda>\bar{\lambda}, \quad \forall k \in[0, \lambda \bar{\tau}[\cap \mathbb{N} .
$$

Proof. From the minimality of $w(\lambda, k+1)$ it follows that

$$
\frac{\lambda}{2}\|w(\lambda, k+1)-w(\lambda, k)\|_{2}^{2} \leq \mathcal{F}(w(\lambda, k))-\mathcal{F}(w(\lambda, k+1)) .
$$

Therefore

$$
\sum_{k_{0} \leq k \leq k_{0}+K}\|w(\lambda, k+1)-w(\lambda, k)\|_{2}^{2} \leq \frac{2}{\lambda} \mathcal{F}(\bar{u}), \quad k_{0}, K \in \mathbb{N} .
$$

From (5.28) and the Hölder inequality we get

$$
\left\|w(\lambda, k)-w\left(\lambda, k_{0}\right)\right\|_{2} \leq\left(\frac{2 K \mathcal{F}(\bar{u})}{\lambda}\right)^{1 / 2}, \quad k_{0}, K \in \mathbb{N}, \quad k_{0} \leq k \leq k_{0}+K .
$$

From Lemma 5.7 (applied with $\bar{u}$ in place of $w$ ) there exists $\bar{\delta}>0$ such that

$$
\|v-\bar{u}\|_{2}<\bar{\delta} \Rightarrow \mathcal{H}^{0}\left(J_{v}\right) \geq \mathcal{H}^{0}\left(J_{\bar{u}}\right)
$$

Using (5.29) with $k_{0}=0$ we get

$$
\|w(\lambda, k)-\bar{u}\|_{2}<\bar{\delta}, \quad \lambda>\bar{\lambda}, \quad k \in[0, \lambda \bar{\tau}[\cap \mathbb{N},
$$

with

$$
\bar{\lambda}:=\frac{2 \mathcal{F}(\bar{u})}{\bar{\delta}^{2}}, \quad \bar{\tau}:=\frac{1}{\bar{\lambda}} .
$$

Then, using (5.30) and Proposition 5.6 (iii), assertion (5.27) follows.

In view of Lemma 5.10 we can give the following definition.

Definition 5.11. We define $\tau_{1} \in[\bar{\tau},+\infty]$ as

$$
\tau_{1}:=\sup \left\{\tau>0: \exists \widetilde{\lambda}>1: w(\lambda, k) \in \Sigma_{1} \quad \forall \lambda>\widetilde{\lambda}, \quad \forall k \in[0, \lambda \tau[\} .\right.
$$




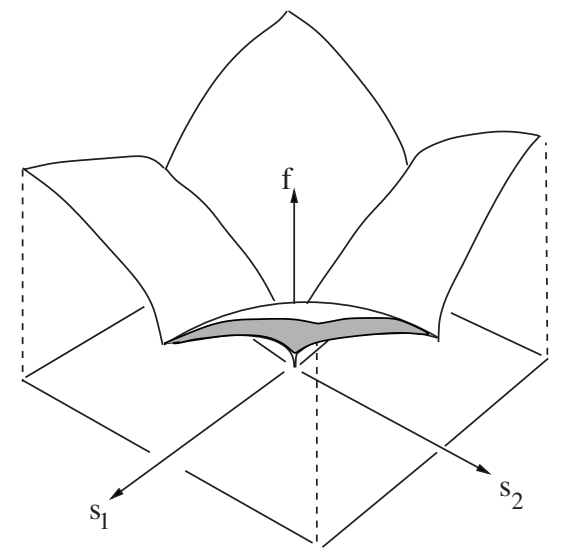

FiguRE 3. $\mathcal{F}$ is not differentiable. For instance, given any $u \in \operatorname{Dom}(\mathcal{F})$ and a point $x$ not in $J_{u}$, the function $\mathcal{F}\left(u+s 1_{[x, \beta[}\right)=\mathcal{F}(u)+\gamma_{a}|s|^{r}$ is not differentiable at $s=0$. If $u \in \mathcal{P}_{\text {fin }}(I)$, then the descending slope coincides with the norm of the gradient of the restriction of $\mathcal{F}$ to the set $\left\{v \in \mathcal{P}_{\text {fin }}(I): J_{v}=J_{u}\right\}$. As an illustration of the singularities of $\mathcal{F}$, we depict the graph of $f\left(s_{1}, s_{2}\right):=\mathcal{F}\left(s_{1} 1_{\left[x_{1}, \beta[\right.}+s_{2} 1_{\left[x_{2}, \beta[\right.}\right)$ for $x_{1} \neq x_{2}$.

Remark 5.12. From (5.27) and standard arguments from the theory of minimizing movements [3], [30, 6] it follows that the $\operatorname{limit}_{\lambda \rightarrow+\infty} w(\lambda,[\lambda \tau])$ exists in $L^{2}(I)$ for all $\tau \in\left[0, \tau_{1}\left[\right.\right.$. Moreover, $\lim _{\lambda \rightarrow+\infty} w(\lambda,[\lambda \cdot]):\left[0, \tau_{1}\left[\rightarrow \Sigma_{1}\right.\right.$ is $\frac{1}{2}$-Hölder continuous and it is the solution of the initial value problem

$$
\left.\dot{u}=-\nabla \mathcal{F}^{1}(u) \quad \text { on }\right] 0, \tau_{1}[, \quad u(0)=\bar{u},
$$

where $\mathcal{F}^{1}$ denotes the restriction of $\mathcal{F}$ to $\Sigma_{1}$, and $\nabla \mathcal{F}^{1}$ is the gradient of $\mathcal{F}^{1}$ with respect to the inner product $\langle\cdot, \cdot\rangle_{1}$ given by

$$
\langle v, w\rangle_{1}=\sum_{h=1}^{N}\left(\sum_{j=0}^{h} s_{j}\right)\left(\sum_{j=0}^{h} \sigma_{j}\right)\left(\bar{x}_{h+1}-\bar{x}_{h}\right),
$$

where $v=s_{0}+\sum_{j=1}^{N} s_{j} 1_{\left[\bar{x}_{j}, \beta[\right.}, w=\sigma_{0}+\sum_{j=1}^{N} \sigma_{j} 1_{\left[\bar{x}_{j}, \beta[\right.}$.

We now prove b) for $i=1$, by showing that (5.33) is indeed equivalent to the ODE (5.4) for the jumps $s_{i}$ of $u$. Let $g:=\nabla \mathcal{F}^{1}(u), g=g_{0}+\sum_{j=1}^{N} g_{j} 1_{\left[\bar{x}_{j}, \beta[\right.}$; then $g$ is determined by the expression

$$
\begin{array}{r}
\left\langle\nabla \mathcal{F}^{1}\left(s_{0}+\sum_{j=1}^{N} s_{j} 1_{\left[\bar{x}_{j}, \beta\right]}\right), \hat{s}_{0}+\sum_{j=1}^{N} \hat{s}_{j} 1_{\left[\bar{x}_{j}, \beta\right]}\right\rangle_{1}=\gamma_{a} \sum_{j=1}^{N} \mathrm{e}_{a}^{\prime}\left(\left|s_{j}\right|\right) \frac{s_{j}}{\left|s_{j}\right|} \hat{s}_{j} \\
=\sum_{h=1}^{N}\left(\sum_{j=0}^{h} g_{j}\right)\left(\sum_{j=0}^{h} \hat{s}_{j}\right)\left(\bar{x}_{h+1}-\bar{x}_{h}\right) .
\end{array}
$$

Taking $\hat{s}_{k}=1, \hat{s}_{j}=0$ for $j \neq k$, from (5.35) we get

$$
\sum_{h=k}^{N}\left(\sum_{j=0}^{h} g_{j}\right)\left(\bar{x}_{h+1}-\bar{x}_{h}\right)=\gamma_{a} \mathrm{e}_{a}^{\prime}\left(\left|s_{k}\right|\right) \frac{s_{k}}{\left|s_{k}\right|}, \quad k=0, \ldots, N .
$$


Subtracting from (5.36) the one corresponding to $k+1$, we obtain

$$
g_{0}\left(\bar{x}_{1}-\bar{x}_{0}\right)=-\gamma_{a} \mathrm{e}_{a}^{\prime}\left(\left|s_{1}\right|\right) \frac{s_{1}}{\left|s_{1}\right|}
$$

and for $k=1, \ldots, N-1$,

$$
\left(\sum_{j=0}^{k} g_{j}\right)\left(\bar{x}_{k+1}-\bar{x}_{k}\right)=\gamma_{a} \mathrm{e}_{a}^{\prime}\left(\left|s_{k}\right|\right) \frac{s_{k}}{\left|s_{k}\right|}-\gamma_{a} \mathrm{e}_{a}^{\prime}\left(\left|s_{k+1}\right|\right) \frac{s_{k+1}}{\left|s_{k+1}\right|},
$$

while for $k=N$ equality (5.35) becomes

$$
\left(\sum_{j=0}^{N} g_{j}\right)\left(\bar{x}_{N+1}-\bar{x}_{N}\right)=\gamma_{a} \mathrm{e}_{a}^{\prime}\left(\left|s_{N}\right|\right) \frac{s_{N}}{\left|s_{N}\right|} .
$$

The above equations uniquely determine $\sum_{j=0}^{k} g_{j}$; hence system (5.33) can be explicitly written as

$$
\left\{\begin{array}{l}
\dot{s}_{0}=-g_{0}=\frac{1}{\bar{x}_{1}-\alpha} \mathrm{e}_{a}^{\prime}\left(\left|s_{1}\right|\right) \frac{s_{1}}{\left|s_{1}\right|}, \\
\frac{d}{d \tau} \sum_{j=0}^{k} s_{j}=-\sum_{j=0}^{k} g_{j}=-\frac{1}{\bar{x}_{k+1}-\bar{x}_{k}}\left(\mathrm{e}_{a}^{\prime}\left(\left|s_{k}\right|\right) \frac{s_{k}}{\left|s_{k}\right|}-\mathrm{e}_{a}^{\prime}\left(\left|s_{k+1}\right|\right) \frac{s_{k+1}}{\left|s_{k+1}\right|}\right), \\
k=1, \ldots, N-1, \\
\frac{d}{d \tau} \sum_{j=0}^{N} s_{j}=-\sum_{j=0}^{N} g_{j}=-\frac{1}{\beta-\bar{x}_{N}} \mathrm{e}_{a}^{\prime}\left(\left|s_{N}\right|\right) \frac{s_{N}}{\left|s_{N}\right|} .
\end{array}\right.
$$

This triangular system can be solved for the $\dot{s}_{i}$, thus yielding (5.4).

To prove (a3) in Theorem 5.4, we observe that, as a consequence of (5.37), (5.38), (5.39), we have

$$
\int_{I} g d x=\sum_{k=0}^{N}\left(\sum_{j=0}^{k} g_{j}\right)\left(\bar{x}_{k+1}-\bar{x}_{k}\right)=0 .
$$

Lemma 5.13. We have $\tau_{1}<+\infty$.

Proof. For $1<h<k<N$, (5.40) implies

$$
\begin{aligned}
\frac{d}{d \tau} \sum_{h \leq j \leq k} s_{j}= & -\frac{1}{\bar{x}_{k+1}-\bar{x}_{k}}\left(e_{a}^{\prime}\left(\left|s_{k}\right|\right) \frac{s_{k}}{\left|s_{k}\right|}-e_{a}^{\prime}\left(\left|s_{k+1}\right|\right) \frac{s_{k+1}}{\left|s_{k+1}\right|}\right) \\
& +\frac{1}{\bar{x}_{h}-\bar{x}_{h-1}}\left(e_{a}^{\prime}\left(\left|s_{h-1}\right|\right) \frac{s_{h-1}}{\left|s_{h-1}\right|}-e_{a}^{\prime}\left(\left|s_{h}\right|\right) \frac{s_{h}}{\left|s_{h}\right|}\right) .
\end{aligned}
$$

Assume that $h, k$ are such that $s_{h-1} s_{h}<0, s_{j-1} s_{j}>0$ for $h<j \leq k$, and $s_{k} s_{k+1}<0$. Multiplying (5.42) by $\operatorname{sign}\left(s_{h}\right)=\operatorname{sign}\left(s_{j}\right)=\operatorname{sign}\left(s_{k}\right), h \leq j \leq k$, we obtain

$$
\begin{aligned}
\frac{d}{d \tau} \sum_{h \leq j \leq k}\left|s_{j}\right|= & -\left(\frac{1}{\bar{x}_{k+1}-\bar{x}_{k}}\left(e_{a}^{\prime}\left(\left|s_{k}\right|\right)+e_{a}^{\prime}\left(\left|s_{k+1}\right|\right)\right)\right. \\
& \left.+\frac{1}{\bar{x}_{h}-\bar{x}_{h-1}}\left(e_{a}^{\prime}\left(\left|s_{h-1}\right|\right)+e_{a}^{\prime}\left(\left|s_{h}\right|\right)\right)\right) \leq-\frac{4}{\beta-\alpha} e_{a}^{\prime}\left(\sum_{j=1}^{N}\left|s_{j}\right|\right)
\end{aligned}
$$


where we have used $\left|\bar{x}_{j}-\bar{x}_{i}\right|<\beta-\alpha$ and the fact that $e_{a}^{\prime}$ is decreasing on $] 0,+\infty[$. Inequality (5.43) is valid also when $h=1$ and/or $k=N$ (and when $N=1,2$ ). Therefore

$$
\frac{d}{d \tau} V(u(\tau))<-\frac{1}{\beta-\alpha} e_{a}^{\prime}(V(u(\tau)))<-\frac{4}{\beta-\alpha} e_{a}^{\prime}(V(\bar{u})),
$$

where $V(u(\tau)):=\sum_{j=1}^{N}\left|s_{j}(\tau)\right|$ is the variation of $u(\tau)$. Inequality (5.44) implies $\tau_{1}<+\infty$.

Note that (5.44) shows that, besides $\mathcal{F}$, (5.4)-(5.6) have a second Liapunov functional; we show later in Remark 5.25 that actually (5.4)-(5.6) have also a third Liapunov functional.

The $\frac{1}{2}$-Hölder continuity of $u$ implies the existence of

$$
\bar{u}_{1}:=\lim _{\tau \uparrow \tau_{1}} u(\tau) .
$$

Lemma 5.14. We have

$$
\bar{u}_{1} \in \partial \Sigma_{1}
$$

and therefore

$$
\mathcal{H}^{0}\left(J_{\bar{u}_{1}}\right)<\mathcal{H}^{0}\left(J_{\bar{u}}\right)
$$

Proof. If $\bar{u}_{1} \in \Sigma_{1}$, then Lemma 5.7 (applied with $\bar{u}_{1}$ in place of $w$ ) yields a $\bar{\delta}>0$ such that $\left\|v-\bar{u}_{1}\right\|_{2}<\bar{\delta}$ implies $\mathcal{H}^{0}\left(J_{v}\right) \geq \mathcal{H}^{0}\left(J_{\bar{u}_{1}}\right)$. By an argument similar to the one developed in the proof of Lemma 5.10 we can establish (5.27) for some $\bar{\tau}_{1}>\tau_{1}$ and some $\bar{\lambda}_{1}<+\infty$, thus contradicting the definition of $\tau_{1}$.

5.1. Continuing the proof of Theorem [5.4; the minimizing movement beyond a singularity. We have to show that, independently of the particular sequence $\{w(\lambda, k)\}_{k}$ considered,

$$
\lim _{\lambda \rightarrow+\infty}\left\|w(\lambda,[\lambda \tau])-u_{1}(\tau)\right\|_{2}=0 \quad \forall \tau \in\left[\tau_{1}, \tau_{2}[,\right.
$$

where $u_{1}$ is the function corresponding to the solution of system (5.4) with initial condition $\bar{u}_{1}$ at time $\tau_{1}$, and $\tau_{2}$ is defined as $\tau_{1}$ with $\bar{u}$ replaced by $\bar{u}_{1}$.

Remark 5.15. From the definition (5.32) of $\tau_{1}$, it follows

$$
\forall \tau \in] 0, \tau_{1}\left[\quad \exists \lambda_{\tau}>1: \quad w(\lambda, k) \in \Sigma_{1} \quad \forall \lambda>\lambda_{\tau}, \forall k \in[0, \lambda \tau[\cap \mathbb{N},\right.
$$

independently of the particular sequence $\{w(\lambda, k)\}_{k}$ considered. This observation and the theory of minimizing movements imply that we can also assume $\lambda_{\tau}$ so large that the sequence $\{w(\lambda, k)\}_{k}$ is uniquely determined, provided that $\lambda>\lambda_{\tau}$ and $k \in[0, \lambda \tau[\cap \mathbb{N}$ (see also Proposition $5.6(\mathrm{v})$ ).

For later reference we quote as a lemma some consequences of the above remark.

Lemma 5.16. Let $\eta \in] 0,1\left[\right.$. Then there exist $\left.\mu_{\eta} \in\right] 0, \tau_{1}\left[\right.$ and $\lambda^{\eta}>1$ such that, independently of the particular sequence $\{w(\lambda, k)\}_{k}$ considered,

$$
\mu \in] 0, \mu_{\eta}\left[, \lambda>\lambda^{\eta} \Longrightarrow\left\{\begin{array}{l}
J_{w\left(\lambda,\left[\lambda\left(\tau_{1}-\mu\right)\right]\right)}=J_{\bar{u}}, \\
\left\|w\left(\lambda,\left[\lambda\left(\tau_{1}-\mu\right)\right]\right)-\bar{u}_{1}\right\|_{2}<\eta, \\
\sum_{x_{j} \in J_{\bar{u} \backslash} J_{\bar{u}_{1}}}\left|s_{j}\left(\lambda,\left[\lambda\left(\tau_{1}-\mu\right)\right]\right)\right|<\eta h_{\bar{u}_{1}},
\end{array}\right.\right.
$$


where $w(\lambda, k)=s_{0}(\lambda, k)+\sum_{x_{j} \in J(w(\lambda, k))} s_{j}(\lambda, k) 1_{\left[x_{j} \beta[\right.}$ and we recall that $h_{\bar{u}_{1}}$ is defined in (5.8).

For any $\mu \in] 0, \tau_{1}[$ write

$\omega_{\lambda, \mu}(k):=w\left(\lambda,\left[\lambda\left(\tau_{1}-\mu\right)\right]+k\right)=s_{0}^{k}(\lambda, \mu)+\sum_{x \in J_{\omega_{\lambda, \mu}(k)}} s_{x}^{k}(\lambda, \mu) 1_{[x, \beta[}, \quad k \in \mathbb{N} \cup\{0\}$, where $s_{x}^{k}(\lambda, \mu)$ is the jump of $\omega_{\lambda, \mu}(k)$ at the jump point $x \in J_{\omega_{\lambda, \mu}(k)}$.

For any $k \in \mathbb{N} \cup\{0\}$ we denote by $\widehat{\omega}_{\lambda, \mu}(k+1)$ a solution of (5.3) associated with $\omega_{\lambda, \mu}(k+1)$ in the sense of Proposition 5.6 (iv). Since

$$
J_{\widehat{\omega}_{\lambda, \mu}(k+1)} \subseteq J_{\omega_{\lambda, \mu}(k)},
$$

we write

$$
\widehat{\omega}_{\lambda, \mu}(k+1)=\widehat{s}_{0}^{k+1}(\lambda, \mu)+\sum_{x \in J_{\omega_{\lambda, \mu}(k)}} \widehat{s}_{x}^{k+1}(\lambda, \mu) 1_{[x, \beta[},
$$

where we do not exclude that some of the $\widehat{s}_{x}^{k+1}(\lambda, \mu)$ may vanish.

Whenever no confusion is possible, we write $s_{x}^{k}$ for $s_{x}^{k}(\lambda, \mu), \omega(k)$ for $\omega_{\lambda, \mu}(k), \widehat{s}_{x}^{k}$ for $\widehat{s}_{x}^{k}(\lambda, \mu)$ and $\widehat{\omega}(k)$ for $\widehat{\omega}_{\lambda, \mu}(k)$.

Lemma 5.17. For any $\mu \in] 0, \tau_{1}[, \lambda>1$ and $k \in \mathbb{N} \cup\{0\}$ we have

$$
\left|\widehat{s}_{x}^{k+1}(\lambda, \mu)-s_{x}^{k}(\lambda, \mu)\right| \leq 2 \bar{c} \lambda^{-1 / 2} \quad \forall x \in J_{\omega(k)},
$$

where $\bar{c}:=\left(\frac{2 \mathcal{F}(\bar{u})}{l_{\pi}}\right)^{1 / 2}$. Therefore

$$
\left|\sum_{x \in J_{\omega(k+1)}}\right| s_{x}^{k+1}(\lambda, \mu)\left|-\sum_{x \in J_{\omega(k)}}\right| s_{x}^{k}(\lambda, \mu)|| \leq 2 N \bar{c} \lambda^{-1 / 2} .
$$

Proof. From Proposition 5.6(iv) and (5.28) we obtain

$$
\|\widehat{\omega}(k+1)-\omega(k)\|_{2}=\|\omega(k+1)-\omega(k)\|_{2} \leq\left(\frac{2 \mathcal{F}(\bar{u})}{\lambda}\right)^{1 / 2} .
$$

Inclusion (5.48), the fact that the range of $\widehat{\omega}(k+1)$ equals the range of $\omega(k+1)$, and (5.10) imply

$$
l_{\bar{u}}^{1 / 2}\|\widehat{\omega}(k+1)-\omega(k)\|_{\infty} \leq\|\widehat{\omega}(k+1)-\omega(k)\|_{2} .
$$

Therefore, from (5.51),

$$
\|\widehat{\omega}(k+1)-\omega(k)\|_{\infty} \leq\left(\frac{2 \mathcal{F}(\bar{u})}{\lambda l_{\bar{u}}}\right)^{1 / 2} .
$$

From this and (5.48) it follows

$$
\left|\widehat{s}_{x}^{k+1}-s_{x}^{k}\right| \leq 2\|\widehat{\omega}(k+1)-\omega(k)\|_{\infty} \leq 2\left(\frac{2 \mathcal{F}(\bar{u})}{\lambda l_{\bar{u}}}\right)^{1 / 2},
$$

and (5.49) follows.

Remark 5.18. We explicitly note that all the estimates and the constants considered in the following will be independent of the particular sequence $\{w(\lambda, k)\}_{k}$ considered; we do not mention this property in the sequel of the section. 
Lemma 5.19. There exist $\mu_{1}>0$ and $\tau_{0}>0$ satisfying the following property: for any $\mu \in] 0, \mu_{1}\left[\right.$ there exists $\left.\lambda_{\mu} \in\right] 1,+\infty\left[\right.$ such that for any $\lambda>\lambda_{\mu}$ and any $k \in\left[0, \lambda \tau_{0}[\cap \mathbb{N}\right.$ we have

(i) $J_{\bar{u}_{1}} \subseteq J_{\omega_{\lambda, \mu}(k)}$;

(ii) $x \in J_{\bar{u}_{1}} \Longrightarrow\left\|\omega_{\lambda, \mu}(k)-\bar{u}_{1}\right\|_{L^{\infty}\left(\left(x-l_{\bar{u}}, x+l_{\bar{u}}\right)\right)}<\frac{1}{4} h_{\bar{u}_{1}}$.

Proof. Given $\delta>0$, define

$$
\tau_{0}:=\frac{\delta^{2}}{4 \mathcal{F}(\bar{u})}, \quad \lambda_{0}:=\frac{1}{\tau_{0}} .
$$

From (5.29) with $k_{0}=\left[\lambda\left(\tau_{1}-\mu\right)\right]$ and (5.52) it follows

$$
\left.\|\omega(k)-\omega(0)\|_{2}<\frac{\delta}{2}, \quad \mu \in\right] 0, \tau_{1}\left[, \lambda>\lambda_{0}, k \in\left[0, \lambda \tau_{0}[\cap \mathbb{N} .\right.\right.
$$

This and the second equality in (5.47) with $\eta=\delta / 2$ yield

$$
\left.\left\|\omega(k)-\bar{u}_{1}\right\|_{2}<\delta, \quad \mu \in\right] 0, \mu_{\eta}\left[, \lambda>\lambda_{\mu}, k \in\left[0, \lambda \tau_{0}[\cap \mathbb{N},\right.\right.
$$

where

$$
\lambda_{\mu}:=\max \left(\lambda_{0}, \lambda^{\eta}\right)
$$

By reducing the value of $\delta$ if necessary, we can assume that Lemma 5.7 holds (with $\bar{u}_{1}$ in place of $\left.w\right)$. Therefore we have

$$
\left.\mathcal{H}^{0}\left(J_{\omega(k)}\right) \geq \mathcal{H}^{0}\left(J_{\bar{u}_{1}}\right), \quad \mu \in\right] 0, \mu_{\eta}\left[, \lambda>\lambda_{\mu}, k \in\left[0, \lambda \tau_{0}[\cap \mathbb{N},\right.\right.
$$

and (5.21) implies that for each $x \in J_{\bar{u}_{1}}$ there are $\xi^{l}=\xi^{l}(\lambda, \mu) \in I, \xi^{r}=\xi^{r}(\lambda, \mu) \in I$ with

$$
\left.\xi^{l}<x<\xi^{r}, \quad\right] \xi^{l}, \xi^{r}\left[\cap J_{\bar{u}_{1}}=\{x\}, \quad\right] \xi^{l}, \xi^{r}\left[\cap J_{\omega(k)} \neq \emptyset .\right.
$$

Note also that (5.10) implies

$$
l_{\omega(k)} \geq l_{\omega(k-1)} \geq \cdots \geq l_{\omega(0)}=l_{w(\lambda,[\lambda(\tau-\mu)])} \geq l_{\bar{u}} .
$$

Let $y=y^{k, \mu, \lambda} \in J_{\omega(k)}$ be a point such that

$$
|y-x|=\min _{\widetilde{y} \in J_{\omega(k)}}|\widetilde{y}-x| .
$$

Step 1. For all $\mu \in] 0, \mu_{\eta}\left[, \lambda>\lambda_{\mu}\right.$ and $k \in\left[0, \lambda \tau_{0}[\right.$ we have

$$
\left\{\begin{array}{l}
|y-x|<l_{\bar{u}} / 2, \\
\left|\omega(k)(y)-\bar{u}_{1}(y)\right|+\left|\omega(k)\left(y_{-}\right)-\bar{u}_{1}\left(x_{-}\right)\right|<\frac{1}{4}\left|\bar{u}_{1}(x)-\bar{u}_{1}\left(x_{-}\right)\right| .
\end{array}\right.
$$

Let $x^{l}, x^{r}$ be defined by $\left[x^{l}, x^{r}\right] \cap J_{\bar{u}_{1}}=\left\{x^{l}, x, x^{r}\right\}\left(x^{l}=\alpha\right.$ if $x=\min J_{\bar{u}_{1}}, x^{r}=\beta$ if $\left.x=\max J_{\bar{u}_{1}}\right)$, and $y^{l}<y<y^{r}$ be defined by $\left[y^{l}, y^{r}\right] \cap J_{\omega(k)}=\left\{y^{l}, y, y^{r}\right\}$. See Figure 4. For definiteness we consider the case

$$
y \geq x,
$$

the case $y \leq x$ being similar. Then (5.57) and (5.56) imply

$$
x-y^{l} \geq \frac{1}{2}\left(y-y^{l}\right) \geq \frac{l_{\bar{u}}}{2} .
$$

From this and $x-x^{l} \geq l_{\bar{u}}$ it follows that the length of the interval $] z^{l}, x[:=] y^{l}, x[\cap] x^{l}, x[$ is at least $\frac{l_{\pi}}{2}$. Therefore we have from (5.54),

$$
\frac{l_{\bar{u}}}{2}\left|\omega(k)\left(y_{-}\right)-\bar{u}_{1}\left(x_{-}\right)\right|^{2} \leq\left(x-z^{l}\right)\left|\omega(k)\left(y_{-}\right)-\bar{u}_{1}\left(x_{-}\right)\right|^{2}<\delta^{2},
$$




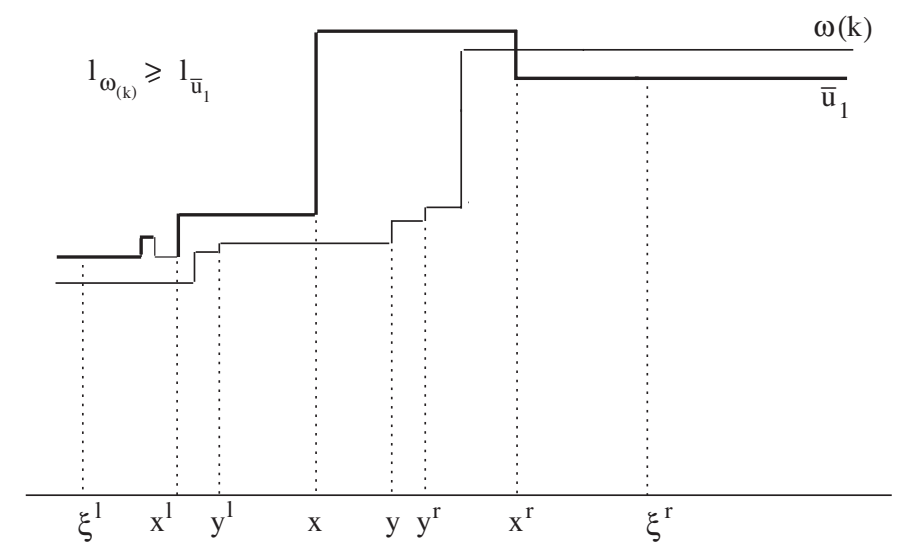

FIGURE 4. The proof of step 1 in Lemma 5.19 In step 2 we prove that $y=x$.

so that

$$
\left|\omega(k)\left(y_{-}\right)-\bar{u}_{1}\left(x_{-}\right)\right|<\sqrt{\frac{2}{l_{\bar{u}}}} \delta,
$$

for all $\mu, \lambda$ and $k$ for which (5.54) is valid.

From $] \xi^{l}, \xi^{r}\left[\cap J_{\bar{u}_{1}}=\{x\}\right.$ it follows $y<x^{r}$. This and (5.59) imply, provided $\delta>0$ is sufficiently small,

$$
\frac{1}{2}(y-x)\left|\bar{u}_{1}(x)-\bar{u}_{1}\left(x_{-}\right)\right|^{2} \leq(y-x)\left|\bar{u}_{1}(x)-\omega(k)\left(y_{-}\right)\right|^{2}<\delta^{2}
$$

hence

$$
y-x<\frac{2 \delta^{2}}{\left|\bar{u}_{1}(x)-\bar{u}_{1}\left(x_{-}\right)\right|^{2}} \leq \frac{l_{\bar{u}}}{2} .
$$

It follows that the length of the interval $] y, z^{r}[:=] y, x^{r}[\cap] y, y^{r}\left[\right.$ is a least $l_{\bar{u}} / 2$. This and (5.54) yield

$$
\frac{l_{\bar{u}}}{2}\left|\omega(k)(y)-\bar{u}_{1}(y)\right|^{2} \leq\left(z^{r}-y\right)\left|\omega(k)(y)-\bar{u}_{1}(y)\right|^{2}<\delta^{2},
$$

and therefore

$$
\left|\omega(k)(y)-\bar{u}_{1}(y)\right|<\sqrt{\frac{2}{l_{\bar{u}}}} \delta .
$$

Then (5.58) follows from (5.60), and provided $\delta>0$ is sufficiently small, from (5.59) and (5.62).

Step 2. We have

$$
\left.y^{k, \mu, \lambda}=x, \quad \forall \mu \in\right] 0, \mu_{\eta}\left[, \forall \lambda>\lambda_{\mu}, \forall k \in\left[0, \lambda \tau_{0}[\cap \mathbb{N} .\right.\right.
$$

We argue by induction on $k$. Since we can assume $\lambda>1$ so large that $\mathcal{H}^{0}\left(J_{\omega(0)}\right)=$ $\mathcal{H}^{0}\left(J_{\bar{u}}\right)$, from Proposition 5.6 (iii) it follows that $J_{\omega(0)}=J_{\bar{u}}$; hence $y^{k-1, \mu, \lambda}=x$ holds for $k=1$. Now let $k>1$ and assume that $y^{k-1, \mu, \lambda}=x$ and $y^{k, \lambda, \mu} \neq x$. Then (5.10) and (5.58) imply that $y^{k, \lambda, \mu} \notin J_{\omega(k-1)}$. Therefore, if we let $\widehat{y} \in J_{\omega(k-1)}$ be defined by $\left.y^{k, \lambda, \mu} \in\right] y^{k-1, \mu, \lambda}, \widehat{y}\left[,\left\{y^{k-1, \mu, \lambda}, \widehat{y}\right\}=\left[y^{k-1, \mu, \lambda}, \widehat{y}\right] \cap J_{\omega(k-1)}\right.$ if 
$y^{k-1, \lambda, \mu}<\widehat{y}$ (and with $y^{k-1, \lambda, \mu}$ and $\widehat{y}$ exchanged if $y^{k-1, \mu, \lambda}>\widehat{y}$ ), from (5.9) and (5.58) we get

$$
\begin{aligned}
|\omega(k)(s)-\omega(k-1)(s)| & =\frac{1}{2}\left|\omega(k)\left(y^{k, \lambda, \mu}\right)-\omega(k)\left(y_{-}^{k, \lambda, \mu}\right)\right| \\
& \left.>\frac{1}{4}\left|\bar{u}_{1}(x)-\bar{u}_{1}\left(x_{-}\right)\right|, \quad s \in\right] y^{k-1, \mu, \lambda}, \widehat{y}[.
\end{aligned}
$$

From this inequality, (5.10) and (5.54) we get (5.64)

$\frac{l_{\bar{u}}}{32}\left|\bar{u}_{1}(x)-\bar{u}_{1}\left(x_{-}\right)\right|^{2} \leq \frac{1}{32}\left|\widehat{y}-y^{k-1, \mu, \lambda}\right|\left|\bar{u}_{1}(x)-\bar{u}_{1}\left(x_{-}\right)\right|^{2} \leq\|\omega(k)-\omega(k-1)\|_{2}^{2} \leq 2 \delta^{2}$.

This contradiction implies $y^{k, \lambda, \mu}=x$. This proves (i). Statement (ii) follows from (i), estimate (5.58) and $\left|x-x^{l}\right| \geq l_{\bar{u}},\left|x-x^{r}\right| \geq l_{\bar{u}},\left|y-y^{l}\right| \geq l_{\bar{u}},\left|y-y^{r}\right| \geq l_{\bar{u}}$.

Definition 5.20. Let $\mu \in] 0, \mu_{1}\left[\right.$ and $\lambda>\lambda_{\mu}$. We define

$\mathcal{K}_{\lambda, \mu}:=\{k \in] 0, \lambda \tau_{0}\left[\cap \mathbb{N}: \mathcal{H}^{0}\left(J_{\omega_{\lambda, \mu}(k+1)}\right)<\mathcal{H}^{0}\left(J_{\omega_{\lambda, \mu}(k)}\right)\right\}=\left\{k_{1}^{\lambda, \mu}, \ldots, k_{\mathcal{M}_{\lambda, \mu}^{\lambda, \mu}}\right\}$, where $k_{h}^{\lambda, \mu}<k_{h+1}^{\lambda, \mu}$ for $h \in\left\{1, \ldots, \mathcal{M}_{\lambda, \mu}-1\right\}$.

Whenever no confusion is possible, we write $k_{h}$ for $k_{h}^{\lambda, \mu}$ and $\mathcal{M}$ for $\mathcal{M}_{\lambda, \mu}$. Set

$$
E(p):=\gamma_{a} r p^{r-1}, \quad p>0,
$$

which coincides with $e_{a}^{\prime}(p)$ for $p>0$ and therefore is a strictly decreasing function diverging to $+\infty$ as $p \rightarrow 0^{+}$.

Proposition 5.21. There exists $\left.\mu_{2} \in\right] 0, \mu_{1}[$ such that

$\forall \mu \in] 0, \mu_{2}\left[\exists \bar{\lambda}_{\mu}>1: \forall \lambda>\bar{\lambda}_{\mu} \Longrightarrow\left\{\begin{array}{l}k_{\mathcal{M}_{\lambda, \mu}} \leq \lambda \tau(\mu, \lambda)<\lambda \tau_{0}, \\ J_{\omega_{\lambda, \mu}(k)}=J_{\bar{u}_{1}} \forall k \in\left[k_{\mathcal{M}_{\lambda, \mu}}+1, \lambda \tau_{0}[\cap \mathbb{N},\right.\end{array}\right.\right.$ where $\tau(\mu, \lambda)$ is a positive continuous function with

$$
\lim _{\mu \rightarrow 0^{+}} \lim _{\lambda \rightarrow+\infty} \tau(\mu, \lambda)=0 .
$$

Proof. We can assume that we are in the conditions to apply Lemma 5.19, in particular, from (i) we have $J_{\bar{u}_{1}} \subseteq J_{\omega(k)}$, and therefore

$$
V^{k}:=\sum_{x \in J_{\omega(k)} \backslash J_{\bar{u}_{1}}}\left|s_{x}^{k}\right|
$$

is well defined. From Lemma 5.17 we have

$$
\left|V^{k_{h}+1}-V^{k_{h}}\right| \leq 2 N \bar{c} \lambda^{-1 / 2}, \quad h \in\{1, \ldots, \mathcal{M}-1\} .
$$

We now estimate $V^{k}$ when $k$ varies between two consecutive indices in $\mathcal{K}_{\lambda, \mu}, k_{h}+1 \leq$ $k \leq k_{h+1}$. By Proposition [5.6 (iii), for $k$ in this interval the set $J_{\omega(k)}$ is constant and is equal to $J_{\omega\left(k_{h}+1\right)}$. 
Claim. There exists $\left.\mu_{2} \in\right] 0, \mu_{1}[$ such that for any $\mu \in] 0, \mu_{2}\left[\right.$ there exists $\lambda>\lambda_{\mu}$ such that

$$
\begin{aligned}
& V^{k_{1}}-V^{0} \leq-\frac{k_{1}}{\lambda} \\
& V^{k_{h+1}}-V^{k_{h}+1} \leq-\frac{\left(k_{h+1}-k_{h}-1\right)}{\lambda} \quad \forall h \in\{1, \ldots, \mathcal{M}-1\} .
\end{aligned}
$$

We will prove only (5.71), since the proof of (5.72) is similar. If $k_{1}=0$ there is nothing to prove in (5.71). Therefore, we can assume $k_{1}>0$.

We have $J_{\omega(k+1)}=J_{\omega(0)}\left(=J_{\bar{u}}\right)$ for $k \in\left\{0, \ldots, k_{1}\right\}$; hence, using the minimality of $\omega(k+1)$, it follows that $\omega(k+1)$ satisfies the Euler-Lagrange equation

$$
\omega(k+1)-\omega(k)=-\frac{1}{\lambda} \nabla \mathcal{F}_{\lambda, \mu}(\omega(k+1)),
$$

where $\mathcal{F}_{\lambda, \mu}$ denotes the restriction of $\mathcal{F}$ to the subspace of $\mathcal{P}_{\text {fin }}(I)$ of the functions $w$ of the form $w=\sigma_{0}+\sum_{x \in J_{\bar{u}}} \sigma_{x} 1_{[x, \beta[}$ (also represented as in (5.26)). Define

$$
\mathcal{I}^{\dagger}:=\left\{j: \bar{x}_{j} \in J_{\bar{u}} \backslash J_{\bar{u}_{1}}\right\} .
$$

To prove (5.71) we begin by making a partition of the set $\mathcal{I}^{\dagger}$ into equivalence classes as follows.

Let $i, j \in \mathcal{I}^{\dagger}$; we say that $i$ and $j$ are equivalent if either $i=j$ or, if $i \neq j$, then

$$
\{\min (i, j), \ldots, \max (i, j)\} \subseteq \mathcal{I}^{\dagger},
$$

and

$$
s_{l}^{k} s_{l+1}^{k}>0 \quad \forall l \in\{\min (i, j), \ldots, \max (i, j)-1\} .
$$

We denote by $[i]$ the equivalence class of $i$.

That is, the set of "small" jumps is subdivided into maximal subgroups of jumps having the same sign, and each subgroup has on the left and/or on the right either one of the extrema of $I$, or a "large" jump, or the extremum of another subgroup.

We also define

$$
i_{-}:=\min [i], \quad i_{+}:=\max [i],
$$

and we say that $\operatorname{sign}[i]=+($ resp. $\operatorname{sign}[i]=-)$ if $s_{h}^{k}>0\left(\right.$ resp. if $\left.s_{h}^{k}<0\right)$ in (5.74) .

We define the total variation $V^{k}([i])$ at step $k$ of the equivalence class $[i]$ as

$$
V^{k}([i]):=\sum_{j=i_{-}}^{i_{+}}\left|s_{j}^{k}\right|, \quad k \in\left\{1, \ldots, k_{1}\right\} .
$$

From (5.73) and the explicit expression (5.37)-(5.39) of the gradient of $\mathcal{F}_{\lambda, \mu}$ it follows that for all $k \in\left\{0, \ldots, k_{1}-1\right\}$ we have

$$
\lambda\left(V^{k+1}([i])-V^{k}([i])\right)=-\frac{E\left(\left|s_{i_{+}}^{k+1}\right|\right)}{\bar{x}_{i_{+}+1}-\bar{x}_{i_{+}}}-\frac{E\left(\left|s_{i_{-}}^{k+1}\right|\right)}{\bar{x}_{i_{-}}-\bar{x}_{i_{-}-1}}+\mathcal{E}^{+}+\mathcal{E}^{-},
$$

where

$$
\mathcal{E}^{+}= \begin{cases}\operatorname{sign}[i] \operatorname{sign}\left(s_{i_{+}+1}^{k+1}\right) \frac{E\left(\left|s_{i_{+}+1}^{k+1}\right|\right)}{\bar{x}_{i_{+}+1}-\bar{x}_{i_{+}}} & \text {if } i_{+}<N \\ 0 & \text { if } i_{+}=N\end{cases}
$$




$$
\mathcal{E}^{-}= \begin{cases}\operatorname{sign}[i] \operatorname{sign}\left(s_{i_{-}-1}^{k+1}\right) \frac{E\left(\left|s_{i_{-}}^{k+1}\right|\right)}{\bar{x}_{i_{-}}-\bar{x}_{i_{-}-1}} & \text { if } i_{-}>1, \\ 0 & \text { if } i_{-}=1 .\end{cases}
$$

Since $\left|s_{l}^{k+1}\right| \leq V^{k+1}$ and $E$ is decreasing it follows

$$
-\frac{E\left(\left|s_{i_{+}}^{k+1}\right|\right)}{\bar{x}_{i_{+}+1}-\bar{x}_{i_{+}}}-\frac{E\left(\left|s_{i_{-}}^{k+1}\right|\right)}{\bar{x}_{i_{-}}-\bar{x}_{i_{-}-1}} \leq-2 \frac{E\left(V^{k+1}\right)}{\beta-\alpha} \leq-2 \frac{E\left(V^{k}+\bar{c} \lambda^{-1 / 2}\right)}{\beta-\alpha},
$$

where in the last inequality we have used (5.70).

From the expressions of $\mathcal{E}^{ \pm}$in (5.76), (5.77), it follows that $\mathcal{E}^{ \pm} \leq 0$, unless $\operatorname{sign}[i] \operatorname{sign}\left(s_{i_{ \pm} \pm 1}^{k+1}\right)>0$, i.e., unless $i_{ \pm} \pm 1 \in\{1, \ldots, N\} \backslash \mathcal{I}^{\dagger}$. In this case, Lemma 5.19 (ii) and (5.10) imply

$$
\mathcal{E}^{ \pm} \leq \frac{2 E\left(h_{\bar{u}_{1}}\right)}{l_{\bar{u}}}
$$

From (5.78), (5.79) we deduce, summing over $[i]$,

$$
\lambda\left(V^{k+1}-V^{k}\right) \leq-2 \frac{E\left(V^{k}+\bar{c} \lambda^{-1 / 2}\right)}{\beta-\alpha}+\frac{2 N E\left(h_{\bar{u}_{1}}\right)}{l_{\bar{u}}} .
$$

We can assume that $\eta$ in Lemma 5.16 has been fixed so small that the right hand side of (5.80), with $k=0$ (provided $\mu \in] 0, \mu_{\eta}\left[\right.$ and $\lambda>\lambda^{\eta}$ ) satisfies

$$
-2 \frac{E\left(V^{0}+\bar{c} \lambda^{-1 / 2}\right)}{\beta-\alpha}+\frac{2 N E\left(h_{\bar{u}_{1}}\right)}{l_{\bar{u}}} \leq-1,
$$

so that $V^{1}<V^{0}$. By induction, this and (5.80) yield

$$
\lambda\left(V^{k+1}-V^{k}\right) \leq-1 .
$$

Summing over $k \in\left\{0, \ldots, k_{1}-1\right\}$, inequality (5.71) follows.

From the claim, (5.70) and an induction argument it follows

$$
V^{k_{h}+1} \leq V^{0}+h \frac{\bar{c}}{\lambda^{1 / 2}} \leq V^{0}+N \frac{\bar{c}}{\lambda^{1 / 2}} .
$$

From this and the claim we deduce

which implies

$$
V^{k_{\mathcal{M}}} \leq V^{0}+N \frac{\bar{c}}{\lambda^{1 / 2}}-\frac{\left(k_{\mathcal{M}}-N\right)}{\lambda},
$$

$$
k_{\mathcal{M}} \leq N+\lambda\left(V^{0}+N \frac{\bar{c}}{\lambda^{1 / 2}}\right)
$$

and

$$
V^{k}=0 \text { for } k>k_{\mathcal{M}} .
$$

The inequalities on the right hand side of (5.67) follow with

$$
\tau(\mu, \lambda):=\frac{N}{\lambda}+V^{0}+N \frac{\bar{c}}{\lambda^{1 / 2}} .
$$

Then (5.68) is a consequence of this expression for $\tau(\mu, \lambda)$ and

$$
\lim _{\lambda \rightarrow+\infty} V^{0}=\sum_{j \in \mathcal{I}^{\dagger}}\left|s_{j}\left(\tau_{1}-\mu\right)\right| .
$$

Finally, the equality $J_{\omega(k)}=J_{\bar{u}_{1}}$ is a consequence of (5.68) and (5.83). 
Definition 5.22. Define

$$
\bar{k}_{\lambda}:=\min \left\{k \in \mathbb{N}: J_{w(\lambda, k)}=J_{\bar{u}_{1}}\right\} .
$$

Recalling the definition of $k_{\mathcal{M}_{\lambda, \mu}}$ in (5.65), it follows

$$
\bar{k}_{\lambda}=\left[\lambda\left(\tau_{1}-\mu\right)\right]+k_{\mathcal{M}_{\lambda, \mu}}+1 .
$$

Lemma 5.23. We have

$$
\begin{aligned}
& \lim _{\lambda \rightarrow+\infty}\left\|\bar{u}_{1}-w\left(\lambda, \bar{k}_{\lambda}\right)\right\|_{2}=0, \\
& \lim _{\lambda \rightarrow+\infty}\left\|w\left(\lambda, \bar{k}_{\lambda}+\left[\lambda\left(\tau-\tau_{1}\right)\right]\right)-w(\lambda,[\lambda \tau])\right\|_{2}=0 \quad \forall \tau \geq \tau_{1} .
\end{aligned}
$$

Proof. Fix $\varepsilon>0$. Write

$$
\begin{aligned}
\left\|\bar{u}_{1}-w\left(\lambda, \bar{k}_{\lambda}\right)\right\|_{2} \leq & \left\|w\left(\lambda, \bar{k}_{\lambda}\right)-w\left(\lambda,\left[\lambda\left(\tau_{1}-\mu\right)\right]\right)\right\|_{2} \\
& +\left\|w\left(\lambda,\left[\lambda\left(\tau_{1}-\mu\right)\right]\right)-u\left(\tau_{1}-\mu\right)\right\|_{2}+\left\|u\left(\tau_{1}-\mu\right)-\bar{u}_{1}\right\|_{2} \\
=: & \text { I }+ \text { II }+ \text { III. }
\end{aligned}
$$

Since III converges to zero as $\mu \rightarrow 0^{+}$by the definition of $\bar{u}_{1}$, we can choose $\bar{\mu}>0$ in such a way that

From (5.86) and (5.28) it follows

$$
\left.\mathrm{III}<\frac{\varepsilon}{3} \quad \forall \mu \in\right] 0, \bar{\mu}[.
$$

$$
\mathrm{I} \leq(2 \mathcal{F}(\bar{u}))^{1 / 2}\left(\frac{k_{\mathcal{M}}+1}{\lambda}\right)^{1 / 2}
$$

This, (5.67) and (5.68) imply that we can fix $\mu \in] 0, \bar{\mu}[$ in such a way that

$$
\mathrm{I}<\frac{\varepsilon}{3}
$$

for $\lambda$ sufficiently large. Finally, observe that for $\lambda>1$ sufficiently large we have II $<\varepsilon / 3$. Therefore I + II + III $<\varepsilon$, and (5.87) follows.

Now write $\left[\lambda\left(\tau-\tau_{1}\right)\right]=[\lambda \tau]-\left[\lambda\left(\tau_{1}-\mu\right)\right]-[\lambda \mu]+\sigma$, where $|\sigma| \leq 2$. Using also (5.86) we have

$$
\begin{aligned}
& \left\|w\left(\lambda, \bar{k}_{\lambda}+\left[\lambda\left(\tau-\tau_{1}\right)\right]\right)-w(\lambda,[\lambda \tau])\right\|_{2} \\
& =\left\|w\left(\lambda,[\lambda \tau]+k_{\mathcal{M}}+1-[\lambda \mu]+\sigma\right)-w(\lambda,[\lambda \tau])\right\|_{2} \\
& \leq \frac{(2 \mathcal{F}(\bar{u}))^{1 / 2}}{\lambda^{1 / 2}}\left(k_{\mathcal{M}}+1+|\sigma|\right)^{1 / 2} .
\end{aligned}
$$

As before, formulas (5.68), (5.67) show that the right hand side of (5.91) is smaller than $\varepsilon$, provided $\mu$ is sufficiently small and $\lambda>1$ sufficiently large, and therefore (5.88) follows.

Lemma 5.24. Let $\Omega \subseteq \mathbb{R}^{n}$ be an open set and $f \in \mathcal{C}^{2}(\Omega)$. For any $\wp \in \Omega$, $k \in \mathbb{N}$ and $\lambda>1$ denote by $\wp(\lambda, k)$ the minimizing sequence originating at $\wp$, in the construction of the minimizing movement for the gradient flow of $f$. Fix $\bar{\wp} \in \Omega$ and $R \in] 0, \operatorname{dist}(\varsigma, \partial \Omega)[$. Then for any $\rho \in] 0, R / 2[$ there exist $T>0$ and $L>0$ independent of $\rho$, and there exists $\bar{\lambda}>1$ such that

$$
\|\wp(\lambda,[\lambda \tau])-\bar{\wp}(\lambda,[\lambda \tau])\| \leq 2 e^{L T}\|\wp-\bar{\wp}\|,
$$

for all $\wp \in \Omega$ with $\|\wp-\bar{\wp}\|<\rho$, for all $\tau \in[0, T]$ and $\lambda>\bar{\lambda}$. 
Proof. Given $\rho \in] 0, R / 2[$ there exists $T(\bar{\wp})>0$ independent of $\rho$ such that if $\wp \in \Omega,\|\wp-\bar{\wp}\|<\rho$, then the solutions to the gradient system of $f$ starting from $\wp$ lie in the fixed ball $B_{R}(\bar{\wp}) \subset \Omega$ centered at $\bar{\wp}$ for any $\tau \in[0,2 T(\bar{\wp})]$. Since under our assumption on $f$ the minimizing movement converges to the solution to the gradient flow system of $f$, for $\lambda$ large enough $\wp(\lambda, k)$ and $\wp(\lambda, k)$ belong to $B_{R}(\bar{\wp})$ for all $k \leq \lambda T(\bar{\wp})$. If $L$ denotes the Lipschitz constant of $\nabla f$ on $B_{R}(\bar{\wp})$, from the minimality property of $\wp(\lambda, \cdot)$ and $\bar{\wp}(\lambda, \cdot)$ it follows

$\wp(\lambda, k+1)-\bar{\wp}(\lambda, k+1)=\wp(\lambda, k)-\bar{\wp}(\lambda, k)-\frac{1}{\lambda}(\nabla f(\wp(\lambda, k+1))-\nabla f(\bar{\wp}(\lambda, k+1)))$.

From (5.93) we get $\|\wp(\lambda, k+1)-\bar{\wp}(\lambda, k+1)\|\left(1-\frac{L}{\lambda}\right) \leq\|\wp(\lambda, k)-\bar{\wp}(\lambda, k)\|$, and therefore

$$
\|\wp(\lambda, k)-\bar{\wp}(\lambda, k)\| \leq\left(1-\frac{L}{\lambda}\right)^{-k}\|\wp-\bar{\wp}\| .
$$

Setting $k=[\lambda \tau]$ for $\tau \in[0, T(\bar{\wp})]$ yields

$$
\|\wp(\lambda,[\lambda \tau])-\bar{\wp}(\lambda,[\lambda \tau])\| \leq\left(1-\frac{L}{\lambda}\right)^{-\frac{\lambda}{L} T(\bar{\wp}) L}\|\wp-\bar{\wp}\| .
$$

If $\lambda$ is sufficiently large we have $\left(1-\frac{L}{\lambda}\right)^{-\frac{\lambda}{L} T(\overline{\zeta)} L} \leq 2 e^{L T(\overline{\wp)})}$, and (5.92) follows.

We are now in a position to conclude the proof of Theorem 5.4.

We apply Lemma 5.24 (taking as initial time $\tau_{1}$ ) with the choice

$$
\bar{\wp}:=\bar{u}_{1}, \quad \wp:=w\left(\lambda, \bar{k}_{\lambda}\right) .
$$

From (5.92) we have that there is $T>0$ such that, for $\lambda$ large, $(5.96)$

$\left\|v\left(\lambda,\left[\lambda\left(\tau-\tau_{1}\right)\right]\right)-w\left(\lambda, \bar{k}_{\lambda}+\left[\lambda\left(\tau-\tau_{1}\right)\right]\right)\right\|_{2} \leq C\left\|\bar{u}_{1}-w\left(\lambda, \bar{k}_{\lambda}\right)\right\|_{2}, \quad \tau \in\left[\tau_{1}, \tau_{1}+T\right]$,

for a suitable constant $C>0$ independent of $\lambda$, where $v(\lambda, k)$ is the sequence corresponding to the minimizing movement of $\mathcal{F}^{2}$ starting from $\bar{u}_{1}$, and $\mathcal{F}^{2}$ is the restriction of $\mathcal{F}$ to the $\left(N_{2}+1\right)$ dimensional set $\Sigma_{2}$ of all functions $f$ of the form $\sigma_{0}+\sum_{x_{j} \in J_{\bar{u}_{1}}} \sigma_{j} 1_{\left[x_{j}, \beta[\right.}$. Recall that the theory of minimizing movement [3] implies that $\lim _{\lambda \rightarrow+\infty}\left\|v\left(\lambda, \lambda\left[\tau-\tau_{1}\right]\right)-u_{1}(\tau)\right\|_{2}=0$ for $\tau \in\left[\tau_{1}, \tau_{2}[\right.$.

From (5.96) and (5.87) we deduce

$$
\lim _{\lambda \rightarrow+\infty}\left\|w\left(\lambda, \bar{k}_{\lambda}+\left[\lambda\left(\tau-\tau_{1}\right)\right]\right)-u_{1}(\tau)\right\|_{2}=0, \quad \tau \in\left[\tau_{1}, \tau_{1}+T\right] .
$$

Then (5.97) and (5.88) imply

$$
\lim _{\lambda \rightarrow+\infty}\left\|w(\lambda,[\lambda \tau])-u_{1}(\tau)\right\|_{2}=0 \quad \forall \tau \in\left[\tau_{1}, \tau_{1}+T\right],
$$

which is the conclusion (5.46) on the interval $\left[\tau_{1}, \tau_{1}+T\right]$. Equation (5.98) can be extended beyond $\tau_{1}+T$ by a further application of Lemma 5.24 with the choice $\bar{\omega}=u_{1}\left(\tau_{1}+T\right)$ and $\omega=w\left(\lambda, \lambda\left[\tau_{1}+T\right]\right)$, and to the whole interval $\left[\tau_{1}, \tau_{2}[\right.$ by successive extensions of this kind.

The previous analysis can be applied in exactly the same way for the discussion of the other singular times $\tau_{i}, i=2, \ldots, M$, by substituting $\mathcal{F}^{1}$ with the restriction 
$\mathcal{F}^{i}$ of $\mathcal{F}$ to the $\left(N_{i}+1\right)$-dimensional set $\Sigma_{i}$ of all functions of the form

$$
v=\sigma_{0}+\sum_{j=1}^{N_{i}} \sigma_{j} 1_{\left[\bar{x}_{j}, \beta\right.},
$$

and $\nabla \mathcal{F}^{i}$ is the gradient of $\mathcal{F}^{i}$ with respect to the inner product $\langle\cdot, \cdot\rangle_{i}$,

$$
\langle v, w\rangle_{i}=\sum_{h=1}^{N_{i}}\left(\sum_{j=0}^{h} s_{j}\right)\left(\sum_{j=0}^{h} \sigma_{j}\right)\left(\bar{x}_{h+1}-\bar{x}_{h}\right),
$$

where $v=s_{0}+\sum_{j=1}^{N_{i}} s_{j} 1_{\left[\bar{x}_{j}, \beta[\right.}, w=\sigma_{0}+\sum_{j=1}^{N_{i}} \sigma_{j} 1_{\left[\bar{x}_{j}, \beta[\right.}$.

Eventually, arguing as in Lemma 5.13, it follows that $\tau_{M}<+\infty$. The proof of Theorem 5.4 is concluded.

Remark 5.25. Besides the functional $\mathcal{F}$, system (5.33) has another Liapunov functional, namely the $L^{2}(I)$-norm of $u$. Indeed,

$$
\begin{aligned}
\frac{1}{2} \frac{d}{d \tau}\|u\|_{2}^{2} & =\sum_{h=0}^{N} \sum_{j=0}^{h} s_{j}\left(\sum_{i=0}^{h} \dot{s}_{i}\right)\left(\bar{x}_{h+1}-\bar{x}_{h}\right) \\
& =\sum_{h=0}^{N}\left(\sum_{j=0}^{h} s_{j}\right)\left(e_{a}^{\prime}\left(\mid s_{h+1}\right) \frac{s_{h+1}}{\left|s_{h+1}\right|}-e_{a}^{\prime}\left(\mid s_{h}\right) \frac{s_{h}}{\left|s_{h}\right|}\right) \\
& =\sum_{k=1}^{N+1}\left(\sum_{j=0}^{k-1} s_{j}\right) e_{a}^{\prime}\left(\left|s_{k}\right|\right) \frac{s_{k}}{\left|s_{k}\right|}-\sum_{k=0}^{N}\left(\sum_{j=0}^{k} s_{j}\right) e_{a}^{\prime}\left(\left|s_{k}\right|\right) \frac{s_{k}}{\left|s_{k}\right|} \\
& =-\sum_{k=1}^{N} s_{k} e_{a}^{\prime}\left(\left|s_{k}\right|\right) \frac{s_{k}}{\left|s_{k}\right|}+\left(\sum_{j=0}^{N} s_{j}\right) e_{a}^{\prime}\left(\left|s_{N+1}\right|\right) \frac{s_{N+1}}{\left|s_{N+1}\right|}-s_{0} e_{a}^{\prime}\left(\left|s_{0}\right|\right) \frac{s_{0}}{\left|s_{0}\right|} \\
& =-\sum_{k=1}^{N} s_{k} e_{a}^{\prime}\left(\left|s_{k}\right|\right) \frac{s_{k}}{\left|s_{k}\right|}=-r \mathcal{F}(u) \leq 0 .
\end{aligned}
$$

Remark 5.26. It is interesting to notice that (5.99) corresponds to an analogous property of (1.10), (1.11), for which it turns out that

$$
\frac{d}{d \tau} \int_{I} u^{2} d x=-\int_{I}\left[\nu^{3}\left(u_{x x}\right)^{2}+\frac{1}{2} \frac{\phi^{\prime}\left(u_{x}\right) u_{x}}{\nu \phi(1 / \nu)}\right] d x \leq 0
$$

5.2. Some examples. Our first example shows that with the help of Proposition 5.6 we can characterize minimizers of (5.3) for simple choices of $w$.

Example 1. Let $w:]-1,1[\rightarrow \mathbb{R}$ be the odd function defined as follows: $w:=0$ in $]-\delta, \delta$ [ and $w:=h>0$ in $] \delta, 1[$, with $\delta \in] 0, \frac{1}{2}[$; see Figure 5. If $\lambda>1$ is sufficiently large, Proposition [5.6 (iv) implies that there is a unique minimizer $v$ of (5.3) of the form $v=s_{0}+s_{1} 1_{[-\delta, 1[}+s_{2} 1_{[\delta, 1[}$. A direct computation shows that $s_{1}=s_{2}=-s_{0}=\widehat{h}$, where $\widehat{h}$ is the unique solution near $h$ of the equation $\frac{r \gamma_{a}}{\widehat{h}^{1-r}}=\lambda(1-\delta)(h-\widehat{h})$, which gives

$$
\widehat{h}=h-\frac{r \gamma_{a}}{\lambda(1-\delta) h^{1-r}}+o(1 / \lambda) .
$$

The next example shows that, depending on the choice of $w$ and $\lambda$, solutions of (5.3) are not necessarily unique. 


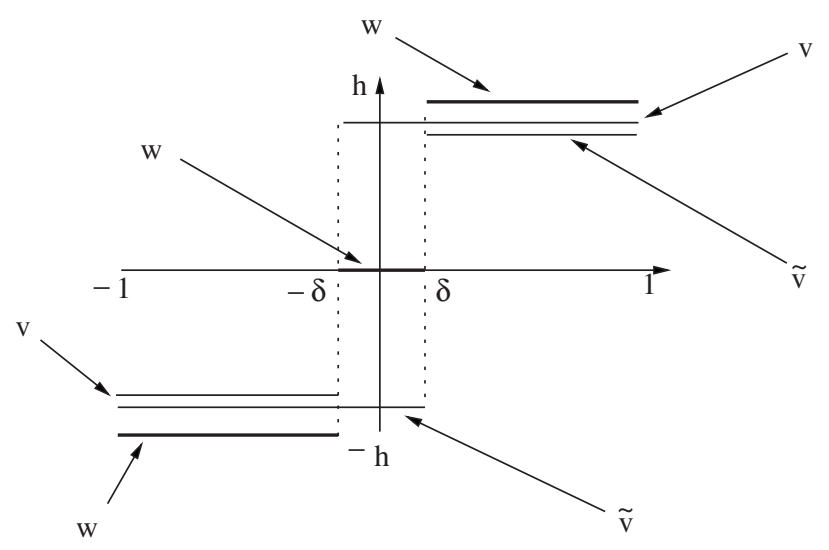

Figure 5. The two minima $v$ and $\widetilde{v}$ of Example 2 .

Example 2. Let $w$ be as in Example 1. Let $v$ be a solution of (5.3). We have $J_{v} \subset$ $[-\delta, \delta]$ because, otherwise, the function $v^{*}$ constructed in the proof of Proposition 5.6 (ii) is such that $\mathcal{G}\left(\lambda, v^{*}, w\right)<\mathcal{G}(\lambda, v, w)$. Hence from Proposition 5.6 (iii) it follows that $v=s_{0}+s_{1} 1_{\left[x_{1}, 1[\right.}+s_{2} 1_{\left[x_{2}, 1[\right.}$ with $-\delta \leq x_{1} \leq x_{2} \leq \delta$. From this and $\delta \in] 0,1 / 2[$ it follows that we can fix $\lambda>0$ sufficiently large to ensure

$$
\max _{x \in]-1,-\delta[\cup] \delta, 1[}|v(x)-w(x)|<h / 2 .
$$

Hence, using the minimality of $v$, it follows that $v$ is nondecreasing, and therefore (5.100) implies

$$
|v(x)-w(x)|=|v(x)|<\frac{3}{2} h, \quad x \in[-\delta, \delta], \quad h<s_{1}+s_{2}<3 h .
$$

Moreover, (5.100) also implies that $v$ is not constant and therefore $1 \leq \mathcal{H}^{0}\left(J_{v}\right) \leq 2$. We claim that there is $\bar{\delta}>0$ such that

$$
\delta \in] 0, \bar{\delta}\left[\Longrightarrow \mathcal{H}^{0}\left(J_{v}\right)=1 .\right.
$$

Assume instead that $x_{1}<x_{2}$ and $s_{j}>0$ for $j=1,2$. We can also suppose $s_{1} \leq s_{2}$ because if $v$ is a minimizer also the function $-v(-\cdot)$ is a minimizer. Set $\widehat{v}:=s_{0}+\left(s_{1}+s_{2}\right) 1_{\left[x_{2}, 1[\right.}$. Then by a computation similar to the one in the proof of Proposition 5.6, we have

$$
\left.|\widehat{v}-w|^{2} \leq(|\widehat{v}-v|+2|v-w|)|\widehat{v}-v| \leq\left(s_{1}+\frac{3}{2} h\right) s_{1} \leq 3 h s_{1} \quad \text { on }\right] x_{1}, x_{2}[.
$$

Therefore

$$
\mathcal{G}(\lambda, v, w)-\mathcal{G}(\lambda, \widehat{v}, w) \geq \gamma_{a}\left(s_{1}^{r}+s_{2}^{r}-\left(s_{1}+s_{2}\right)^{r}\right)-\frac{3}{2} \lambda h s_{1} \delta .
$$

If we let $R(\rho)$ denote the right hand side of (5.104) considered as a function of $\rho:=s_{1}^{r}$, then we have $R(0)=0, R^{\prime}(0)=\gamma_{a}$ independently of $\left.\delta \in\right] 0,1 / 2[$ and $s_{1}+s_{2}>h$. It follows that there is a constant $c>0$ independent of $\delta$ such that

$$
\left.R(\rho)>0 \quad \forall s_{1} \in\right] 0, c[.
$$

On the other hand, $s_{1} \geq c$ implies $\gamma_{a}\left(s_{1}^{r}+s_{2}^{r}-\left(s_{1}+s_{2}\right)^{r}\right) \geq C>0$ for some constant $C$; therefore we conclude that, for $\delta \in] 0, \bar{\delta}\left[, \bar{\delta}:=\frac{4}{9} \frac{C}{\lambda h^{2}}\right.$, the right hand 
side of (5.104) is positive, thus contradicting the minimality of $v$. This proves (5.102).

Once (5.102) is established, the nonuniqueness of $v$ follows from Proposition (5.6) (ii) and the above observation that $-v(-$.$) is also a minimizer. We observe that all$ minimizers $v$ must have $J_{v}=\{-\delta\}$ or $J_{v}=\{\delta\}$. To see this, assume that there is a minimizer $v$ of the form

$$
v=-h+p+(2 h-p-q) 1_{[-\delta+\xi, 1[},
$$

where $\xi \in] 0,2 \delta[$. Then $\mathcal{G}(\lambda, v, w)$ is linear in $\xi$, and in order that $v$ be a minimizer we must have $p=q$. Therefore $\mathcal{G}(\lambda, v, w)$ is independent of $\xi$. This implies the existence of a minimizer of the form (5.106) with $\xi=0$ and $p=q$. In this case the variation of $\mathcal{G}(\lambda, \cdot, w)$ restricted to $2 h-p-q=$ const should vanish for some $p=q$. That is, $(p-h) \delta=0$, which is impossible.

In the next examples we solve explicitly systems (5.4), (5.5) in two particularly simple cases.

Example 3. Assume $a=0, \bar{s}_{0}>0, \bar{s}_{1}>0, \alpha=0, \beta=2, x_{1}=1$, and $\bar{u}=$ $\bar{s}_{0}+\bar{s}_{1} 1_{\left[x_{1}, \beta[\right.}$. Then from (5.5) it follows

$$
\dot{s}_{0}=\frac{\gamma_{0}}{\sqrt{s_{1}}}, \quad \dot{s}_{1}=-\frac{2 \gamma_{0}}{\sqrt{s_{1}}}, \quad s_{i}(0)=\bar{s}_{i}, i=0,1 .
$$

Therefore $s_{0}(\tau)=\bar{s}_{0}+\frac{\bar{s}_{1}}{2}-\frac{1}{2}\left(\bar{s}_{1}^{3 / 2}-3 \gamma_{0} \tau\right)^{2 / 3}$ and $s_{1}(\tau)=\left(\bar{s}_{1}^{3 / 2}-3 \gamma_{0} \tau\right)^{2 / 3}$ for all $\tau \in\left[0, \frac{\bar{s}_{1}^{3 / 2}}{3 \gamma_{0}}\right]$; see Figure 6 a).

Example 4. Assume $a=0, \bar{s}_{1}=-\bar{s}_{0}>0, \bar{s}_{2}=\bar{s}_{1}, \alpha=-1, \beta=1, x_{1}=-\delta$, $x_{2}=\delta$. Then from (5.6) it follows

$$
\left\{\begin{array}{l}
\dot{s}_{0}=\frac{\gamma_{0}}{1-\delta} \frac{s_{1}}{\left|s_{1}\right|^{3 / 2}} \\
\dot{s}_{1}=\frac{\gamma_{0}}{2 \delta} \frac{s_{2}}{\left|s_{2}\right|^{3 / 2}}-\gamma_{0}\left(\frac{1}{1-\delta}+\frac{1}{2 \delta}\right) \frac{s_{1}}{\left|s_{1}\right|^{3 / 2}}, \\
\dot{s}_{2}=-\gamma_{0}\left(\frac{1}{1-\delta}+\frac{1}{2 \delta}\right) \frac{s_{2}}{\left|s_{2}\right|^{3 / 2}}+\frac{\gamma_{0}}{2 \delta} \frac{s_{1}}{\left|s_{1}\right|^{3 / 2}},
\end{array} s_{i}(0)=\bar{s}_{i}, i=1,2,3\right.
$$

Then $s_{1}(\tau)=\left(-\frac{3 \gamma_{0} \tau}{1-\delta}+\bar{s}_{1}^{3 / 2}\right)^{2 / 3}$ and $s_{1}(\tau)=-s_{0}(\tau), s_{2}(\tau)=s_{1}(\tau)$ for all $\tau \in$ $\left[0, \frac{(1-\delta) \bar{s}_{1}^{3 / 2}}{3 \gamma_{0}}\right]$; see Figure 6 b).

\section{Continuity of the minimizing Movement WITH RESPECT TO INITIAL DATA}

The following theorem states that the minimizing movement is continuous with respect to the initial data.

Theorem 6.1. Let $\bar{u} \in \mathcal{P}_{\text {fin }}(I)$. Then, given $\varepsilon>0$, there exists $\delta>0$ such that the conditions

$$
\bar{v} \in \mathcal{P}_{\text {fin }}(I), \quad\|\bar{v}-\bar{u}\|_{2}<\delta, \quad|\mathcal{F}(\bar{v})-\mathcal{F}(\bar{u})|<\delta
$$

imply

$$
\|v(\tau)-u(\tau)\|_{2}<\varepsilon, \quad \tau \in[0,+\infty[,
$$

where $v(\tau)$ (resp. $u(\tau)$ ) denotes the minimizing movement starting from $\bar{v}$ (resp. from $\bar{u})$. 


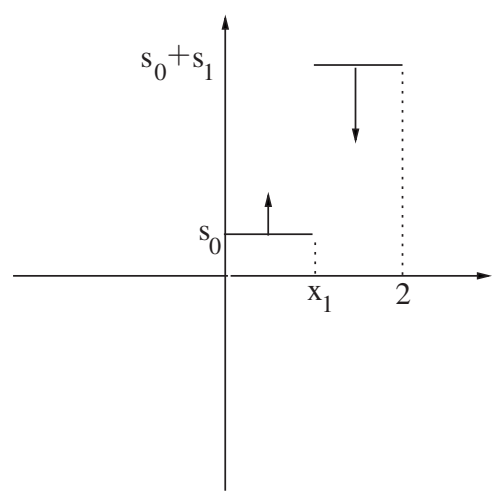

a)

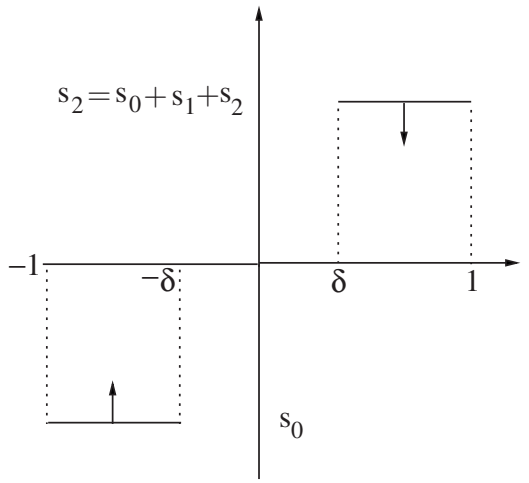

b)

Figure 6. Examples 3 and 4. The arrows indicate the evolution of the function $u(\tau)=s_{0}(\tau)+s_{1}(\tau) 1_{\left[x_{1}, 1[\right.}$ in case a), and $u(\tau)=s_{0}(\tau)+$ $s_{1}(\tau) 1_{[-\delta, 1[}+s_{2}(\tau) 1_{[\delta, 1[}$ in case b).

The proof is based on two lemmata.

Lemma 6.2. Let $w \in \mathcal{P}_{\text {fin }}(I), w=\sigma_{0}+\sum_{h=1}^{M} \sigma_{h} 1_{\left[y_{h}, \beta[\right.}$. Let $\left.\kappa \in\right] 0,1[$. Then we can find $\delta_{\kappa}>0$ such that the conditions

$$
u \in \mathcal{P}_{\text {fin }}, \quad u=s_{0}+\sum_{j=1}^{N} s_{j} 1_{\left[x_{j}, \beta[\right.}, \quad\|u-w\|_{2}<\delta_{\kappa}, \quad|\mathcal{F}(u)-\mathcal{F}(w)|<\delta_{\kappa},
$$

imply the existence of distinct indices $j_{h}, h=1, \ldots, M$, satisfying

$$
\sum_{h=1}^{M}\left|\sigma_{h}-s_{j_{h}}\right|+\sum_{j=1, \ldots, N, j \notin\left\{j_{1}, \ldots, j_{M}\right\}}\left|s_{j}\right|<\kappa \sigma,
$$

where $\sigma:=h_{w}=\min _{h}\left\{\left|\sigma_{h}\right|\right\}$.

Proof. We assume that $\delta$ is so small that Lemma 5.7 is valid. In particular $N \geq M$, and the points $\xi_{h}, h=1, \ldots, M$ are well defined as in the proof of Lemma 5.7 Define $j_{h}$ as follows: $j_{0}:=0$ and

$$
\left\{\begin{array}{l}
\xi_{h-1}<x_{j_{h}} \leq \xi_{h}, \\
\left|s_{j}\right| \leq\left|s_{j_{h}}\right| \quad \forall j: \xi_{h-1}<x_{j} \leq \xi_{h},
\end{array} \quad h=1, \ldots, M\right.
$$

We first prove that

$$
\forall \kappa \in] 0,1\left[\exists \delta_{\kappa}>0:(\underline{6.2}) \Longrightarrow\left|\sigma_{h}-s_{j_{h}}\right|<\kappa \sigma \quad \forall h=1 \ldots, M .\right.
$$

We argue by contradiction. Assume that there exist $\bar{\kappa}>0$ and a sequence $\left\{u^{n}\right\}$, $u^{n}=s_{0}^{n}+\sum_{j=1}^{N_{n}} s_{j}^{n} 1_{\left[x_{j}^{n}, \beta[\right.}$, such that

$$
\lim _{n \rightarrow+\infty}\left(\left\|u^{n}-w\right\|_{2}+\left|\mathcal{F}\left(u^{n}\right)-\mathcal{F}(w)\right|\right)=0,
$$

and $\left|\sigma_{h}-s_{j_{h}}^{n}\right| \geq \bar{\kappa} \sigma$ for some $h \in\{1, \ldots, M\}$. Possibly passing to a subsequence, we can find $\kappa_{1} \geq 0, \ldots, \kappa_{M} \geq 0, \sum_{h=1}^{M} \kappa_{h} \geq \bar{\kappa}$, such that

$$
\left|\sigma_{h}-s_{j_{h}}^{n}\right| \geq \kappa_{h} \sigma \quad \forall h \in\{1, \ldots, M\}, \forall n \in \mathbb{N} \text {. }
$$


For later reference, we observe that we can assume

$$
0<\bar{\kappa} \leq \sum_{h=1}^{M} \kappa_{h} \leq b_{0}
$$

where $b_{0}$ is a given fixed number. From (5.22) and

$$
u^{n}\left(\xi_{h}\right)-u^{n}\left(\xi_{h-1}\right)=\sum_{\xi_{h-1}<x_{j} \leq \xi_{h}} s_{j}^{n}, \quad h=1, \ldots, M,
$$

it follows

$$
\begin{gathered}
\left|\sigma_{h}\right|-\frac{2}{l_{w}^{1 / 2}}\left\|u^{n}-w\right\|_{2} \leq\left|\sum_{\xi_{h-1}<x_{j} \leq \xi_{h}} s_{j}^{n}\right| \\
\kappa_{h} \sigma-\frac{2}{l_{w}^{1 / 2}}\left\|u^{n}-w\right\|_{2} \leq\left|\sigma_{h}-s_{j_{h}}^{n}\right|-\frac{2}{l_{w}^{1 / 2}}\left\|u^{n}-w\right\|_{2} \leq\left|\sum_{\xi_{h-1}<x_{j} \leq \xi_{h}} s_{j}^{n}-s_{j_{h}}\right|,
\end{gathered}
$$$$
\text { where in (6.10) we have also used (6.7). }
$$

To each $h \in\{1, \ldots, M\}$ such that $\kappa_{h}=0$ we associate the inequality (6.11)

$$
\left|\sigma_{h}\right|^{r}-\eta\left(\frac{2}{l_{w}^{1 / 2}}\left\|u^{n}-w\right\|_{2}\right)^{r} \leq\left(\left|\sigma_{h}\right|-\frac{2}{l_{w}^{1 / 2}}\left\|u^{n}-w\right\|_{2}\right)^{r} \leq\left|\sum_{\xi_{h-1}<x_{j} \leq \xi_{h}} s_{j}^{n}\right|^{r},
$$

which follows from (6.9). Using (2.6) and the elementary inequality

$$
B^{r} \leq(B-b)^{r}+\eta b^{r}
$$

which is valid for $0<b<B, 0<\eta<1, b<b_{\eta}$, with $b_{\eta}$ a positive number which depends on $\eta$, from (6.11) we deduce that, for such values of $h$,

$$
\left|\sigma_{h}\right|^{r}-\eta\left(\frac{2}{l_{w}^{1 / 2}}\left\|u^{n}-w\right\|_{2}\right)^{r} \leq \sum_{\xi_{h-1}<x_{j} \leq \xi_{h}}\left|s_{j}^{n}\right|^{r}
$$

If instead $\kappa_{h}>0$ for some $h \in\{1, \ldots, M\}$, denoting by $A_{h}$ (resp. $a_{h}$ ) the left hand side of (6.9) (resp. of (6.10) we have, for $n$ sufficiently large and provided $b_{0}$ in (6.8) is taken sufficiently small, $0<a_{h}<A_{h} / 2$. Observe that, given numbers $0<a<A / 2$, the concavity of the function $p \rightarrow p^{r}$ implies

$$
\begin{aligned}
& \min \left\{\sum_{i=0}^{H} p_{i}^{r}: H \in \mathbb{N}, H \geq 1, \sum_{i=0}^{H} p_{i} \geq A, \sum_{i=1}^{H} p_{i} \geq a, p_{0} \geq \min _{i=1, \ldots, H}\left\{p_{i}\right\}>0\right\} \\
= & \min \left\{p_{0}^{r}+p_{1}^{r}: p_{0}+p_{1}=A, a \leq p_{1} \leq A / 2\right\}=(A-a)^{r}+a^{r} .
\end{aligned}
$$

From this observation and the definition of $a_{h}, A_{h}$, we deduce

$$
\begin{aligned}
& \left|\sigma_{h}\right|+(1-\eta)\left(\kappa_{h} \sigma\right)^{r}-\eta\left(\frac{2}{l_{w}^{1 / 2}}\left\|u^{n}-w\right\|_{2}\right)^{r} \\
\leq & \left(\left|\sigma_{h}\right|-\kappa_{h} \sigma\right)^{r}+\left(\kappa_{h} \sigma-\frac{2}{l_{w}^{1 / 2}}\left\|u^{n}-w\right\|_{2}\right)^{r} \leq \sum_{\xi_{h-1}<x_{j} \leq \xi_{h}}\left|s_{j}^{n}\right|^{r},
\end{aligned}
$$


where we have again made use of (6.12). By adding the $M$-equations (6.11), (6.15), we get

$$
\sum_{h=1}^{M}\left|\sigma_{h}\right|^{r}+(1-\eta) \sum_{h=1}^{M}\left(\kappa_{h} \sigma\right)^{r} \leq \sum_{j}\left|s_{j}^{n}\right|^{r}+\eta M\left(\frac{2}{l_{w}^{1 / 2}}\left\|u^{n}-w\right\|_{2}\right)^{r} .
$$

Therefore, recalling that $\mathcal{F}(w) / \gamma_{a}=\sum_{h=1}^{M}\left|\sigma_{h}\right|^{r}$, we have

$$
\begin{aligned}
\gamma_{a}(1-\eta)\left(\sum_{h=0}^{M} \kappa_{h} \sigma\right)^{r} & \leq \gamma_{a}(1-\eta) \sum_{h=0}^{M}\left(\kappa_{h} \sigma\right)^{r} \\
& \leq \mathcal{F}\left(u^{n}\right)-\mathcal{F}(w)+\eta M \gamma_{a}\left(\frac{2}{l_{w}^{1 / 2}}\left\|u^{n}-w\right\|_{2}\right)^{r} \\
& \leq\left|\mathcal{F}\left(u^{n}\right)-\mathcal{F}(w)\right|+\eta M \gamma_{a}\left(\frac{2}{l_{w}^{1 / 2}}\left\|u^{n}-w\right\|_{2}\right)^{r},
\end{aligned}
$$

which, thanks to (6.6), contradicts the assumption that $\sum_{h=1}^{M} \kappa_{h}>\bar{\kappa}$. This concludes the proof of (6.5).

To complete the proof we observe that

$$
\gamma_{a}\left(\sum_{j=1, \ldots, N, j \notin\left\{j_{1}, \ldots, j_{M}\right\}}\left|s_{j}\right|^{r}-\sum_{h=1}^{M}\left(\left|\sigma_{h}\right|^{r}-\left|s_{j_{h}}\right|^{r}\right)\right)=\mathcal{F}(u)-\mathcal{F}(w) .
$$

If $\left|\sigma_{h}\right|-\left|s_{j_{h}}\right|>0$, using (6.12) and (6.5) we can write

$$
\left|\sigma_{h}\right|^{r}-\eta\left(\left|\sigma_{h}\right|-\left|s_{j_{h}}\right|\right)^{r} \leq\left|\sigma_{h}\right|^{r}-\eta(\kappa \sigma)^{r} \leq\left|s_{j_{h}}\right| .
$$

From this and (6.18) it follows

$$
\begin{aligned}
\gamma_{a}\left(\sum_{j=1, \ldots, N, j \notin\left\{j_{1}, \ldots, j_{M}\right\}}\left|s_{j}\right|\right)^{r} & \leq \gamma_{a} \sum_{j=1, \ldots, N, j \notin\left\{j_{1}, \ldots, j_{M}\right\}}\left|s_{j}\right|^{r} \\
& \leq \mathcal{F}(u)-\mathcal{F}(w)+\eta M(\kappa \sigma)^{r} .
\end{aligned}
$$

This, together with (6.5), implies (6.2), and concludes the proof.

The next lemma is a generalization of inequality (5.99) and can be considered as a proof that the gradient flow defined by $\mathcal{F}$ has a regularizing effect. Roughly speaking, this lemma says that $u(\tau)$ always tries to reduce its distance from a suitable defined step average of $u(\tau)$ which is naturally regarded as a regularization of $u(\tau)$. To state the lemma we introduce the operator $u \in \mathcal{P}_{\text {fin }}(I) \rightarrow z_{u} \in \mathcal{P}_{\text {fin }}(I)$ defined for $u$ sufficiently close to $\bar{v}$ in the sense of Lemma 6.2 so that $j_{h}, h=$ $0, \ldots, M$ are well defined:

$$
\left\{\begin{array}{l}
z_{u}:=\widehat{\sigma}_{0}+\sum_{h=1}^{M} \widehat{\sigma}_{h} 1_{\left[x_{j_{h}}, \beta[\right.}, \\
\sum_{k=0}^{h} \widehat{\sigma}_{k}:=\frac{1}{x_{j_{h+1}}-x_{j_{h}}} \int_{x_{j_{h}}}^{x_{j_{h+1}}} u d x, \quad h=0, \ldots, M, \quad x_{j_{0}}:=\alpha, x_{j_{M+1}}:=\beta .
\end{array}\right.
$$

In the following lemma the function $u$ denotes the minimizing movement starting at time zero from a given initial datum in $\mathcal{P}_{\text {fin }}(I)$, and the derivatives in (6.20), (6.24) below are computed at $\tau=0$. 
Lemma 6.3. Let $u=s_{0}+\sum_{j=1}^{N} s_{j} 1_{\left[x_{j}, \beta[\right.} \in \mathcal{P}_{\text {fin }}(I)$ be in a neighbourhood of $w$ as in (6.2). Then

$$
\frac{d}{d \tau} \frac{1}{2}\left\|u-z_{u}\right\|_{2}^{2} \leq-\sum_{j=1, \ldots, N, j \neq j_{h}, h=1, \ldots, M} s_{j}\left(q_{j}-\widetilde{q}_{j}^{h}\right),
$$

where

$$
\left\{\begin{array}{l}
q_{l}:=\mathrm{e}_{a}^{\prime}\left(\left|s_{l}\right|\right) \frac{s_{l}}{\left|s_{l}\right|}, \quad 0<l \leq N, \\
q_{0}=q_{N+1}=q_{j_{0}}=q_{j_{M+1}}:=0,
\end{array}\right.
$$

and

$\widetilde{q}_{j}^{h}=q_{j_{h}}-q_{j_{h+1}}+\frac{1}{x_{j_{h+1}}-x_{j_{h}}}\left[\left(x_{j_{h+1}}-x_{j}\right) q_{j_{h}}+\left(x_{j}-x_{j_{h}}\right) q_{j_{h+1}}\right], x_{j_{h}}<x_{j}<x_{j_{h+1}}$.

Moreover, given $\lambda \in] 0,1[$, if $\kappa$ in Lemma 6.2 is sufficiently small, we have

$$
\left|\widetilde{q}_{j}^{h}\right|<\lambda q_{j}, \quad \forall j
$$

and

$$
\frac{d}{d \tau} \frac{1}{2}\left\|u-z_{u}\right\|_{2}^{2} \leq-C\left\|u-z_{u}\right\|_{2}^{r}
$$

with $C=\gamma_{a} \frac{r(1-\lambda)}{2^{r}|I|^{r / 2}}$.

Proof. Set for simplicity $z:=z_{u}$ and $z_{h}$ the constant value (in space) of $z_{\mid]} x_{j_{h}}, x_{j_{h+1}}$, and observe that $\dot{z}=z_{\dot{u}}$. We compute

$$
\frac{1}{2} \frac{d}{d \tau}\|u-z\|_{2}^{2}=\langle u-z, \dot{u}-\dot{z}\rangle=\langle u, \dot{u}\rangle-\langle z, \dot{z}\rangle,
$$

where $\langle\cdot, \cdot\rangle$ is the $L^{2}(I)$-scalar product, and we have used the following facts:

$$
\begin{aligned}
\langle z, \dot{u}\rangle & =\sum_{h=0}^{M} \int_{x_{j_{h}}}^{x_{j_{h+1}}} z \dot{u} d x=\sum_{h=0}^{M} z_{h} \frac{d}{d \tau} \int_{x_{j_{h}}}^{x_{j_{h+1}}} u d x \\
& =\sum_{h=0}^{M} z_{h} \dot{z}_{h}\left(x_{j_{h+1}}-x_{j_{h}}\right)=\langle z, \dot{z}\rangle,
\end{aligned}
$$

and

$$
\langle u, \dot{z}\rangle=\sum_{h=0}^{M} \int_{x_{j_{h}}}^{x_{j_{h+1}}} u \dot{z}_{h}=\sum_{h=0}^{M} z_{h} \dot{z}_{h}\left(x_{j_{h+1}}-x_{j_{h}}\right)=\langle z, \dot{z}\rangle .
$$

Observe now that (5.40) can be rewritten as

$$
\sum_{j=0}^{l} \dot{s}_{j}=\frac{1}{x_{l+1}-x_{l}}\left(q_{l+1}-q_{l}\right), \quad l=0, \ldots, N,
$$


where we adopt the notation in (6.21), with $x_{0}:=\alpha, x_{N+1}=\beta$, and we set $j_{0}:=0$, $j_{M+1}:=N+1$. Since, for $h=0, \ldots, M$,

$$
\left\{\begin{array} { l } 
{ z = \frac { 1 } { x _ { j _ { h + 1 } } - x _ { j _ { h } } } \sum _ { i = 0 } ^ { j _ { h + 1 } - j _ { h } - 1 } ( x _ { j _ { h } + 1 + i } - x _ { j _ { h } + i } ) \sum _ { j = 0 } ^ { j _ { h } + i } s _ { j } , } \\
{ \dot { z } = \frac { 1 } { x _ { j _ { h } + 1 } - x _ { j _ { h } } } \sum _ { i = 0 } ^ { j _ { h + 1 } - j _ { h } - 1 } ( x _ { j _ { h } + 1 + i } - x _ { j _ { h } + i } ) \sum _ { j = 0 } ^ { j _ { h } + i } \dot { s } _ { j } , }
\end{array} \text { on } \left[x_{j_{h}}, x_{j_{h+1}}[,\right.\right.
$$

from (6.28) it follows

$$
\begin{aligned}
\dot{z} & =\frac{1}{x_{j_{h+1}}-x_{j_{h}}} \sum_{i=0}^{j_{h+1}-j_{h}-1}\left(q_{j_{h}+i+1}-q_{j_{h}+i}\right) \\
& =\frac{1}{x_{j_{h+1}}-x_{j_{h}}}\left(q_{j_{h}+1}-q_{j_{h}}\right), \quad \text { on }\left[x_{j_{h}}, x_{j_{h+1}}[.\right.
\end{aligned}
$$

Therefore

$$
\begin{aligned}
\langle z, \dot{z}\rangle= & \sum_{h=0}^{M}\left(x_{j_{h}+1}-x_{j_{h}}\right) z_{h} \dot{z}_{h} \\
= & \sum_{h=0}^{M} \sum_{i=0}^{j_{h+1}-j_{h}-1}\left(q_{j_{h+1}}-q_{j_{h}}\right) \frac{x_{j_{h}+1+i}-x_{j_{h}+i}}{x_{j_{h+1}}-x_{j_{h}}} \sum_{j=0}^{j_{h}} s_{j} \\
& +\sum_{h=0}^{M} \sum_{i=1}^{j_{h+1}-j_{h}-1}\left(q_{j_{h+1}}-q_{j_{h}}\right) \frac{x_{j_{h}+1+i}-x_{j_{h}+i}}{x_{j_{h+1}}-x_{j_{h}}} \sum_{j=j_{h}+1}^{j_{h}+i} s_{j}=: A+B .
\end{aligned}
$$

If we set

$$
\sum_{j=j_{k}+1}^{j_{k+1}-1} s_{j}=\widetilde{s}_{k}
$$

we can write

$$
\sum_{j=0}^{j_{h}} s_{j}=\sum_{k=0}^{h} s_{j_{k}}+\sum_{k=0}^{h-1} \widetilde{s}_{k} .
$$

Using this we rewrite $A$ in the form $A=A_{1}+A_{2}$ where

$$
\begin{aligned}
A_{1} & =\sum_{h=0}^{M} \frac{\left(q_{j_{h+1}}-q_{j_{h}}\right)}{x_{j_{h}+1}-x_{j_{h}}} \sum_{i=0}^{j_{h+1}-j_{h}-1}\left(x_{j_{h}+1+i}-x_{j_{h}+i}\right) \sum_{k=0}^{h} s_{j_{k}} \\
& =\sum_{h=0}^{M}\left(q_{j_{h+1}}-q_{j_{h}}\right) \sum_{k=0}^{h} s_{j_{k}}=\sum_{h=1}^{M+1} q_{j_{h}} \sum_{k=0}^{h-1} s_{j_{k}}-\sum_{h=0}^{M} q_{j_{h}} \sum_{k=0}^{h} s_{j_{k}} \\
& =q_{j_{M+1}} \sum_{k=0}^{h-1} s_{j_{k}}-q_{j_{0}} \sum_{k=0}^{h} s_{j_{k}}+\sum_{h=1}^{M} q_{j_{h}}\left(\sum_{k=0}^{h-1} s_{j_{k}}-\sum_{k=0}^{h} s_{j_{k}}\right)=-\sum_{h=1}^{M} s_{j_{h}} q_{j_{h}},
\end{aligned}
$$

where we have used $q_{j_{0}}=q_{j_{M+1}}=0$. The term $A_{2}$ has the same algebraic structure as $A_{1}$ with $\widetilde{s}_{k}$ replacing $s_{j_{k}}$. Hence

$$
A_{2}=-\sum_{h=1}^{M} \widetilde{s}_{h-1} q_{j_{h}}=-\sum_{h=1}^{M} \sum_{j=j_{h-1}+1}^{j_{h}-1} s_{j} q_{j_{h}}=-\sum_{h=0}^{M-1} \sum_{j=j_{h}+1}^{j_{h+1}-1} s_{j} q_{j_{h}} .
$$




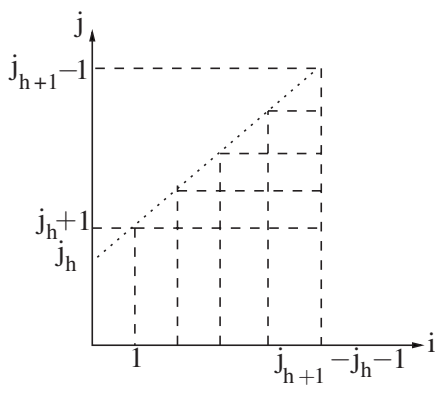

FiguRE 7. The exchange of order of summation in 6.31)

In order to derive a useful expression for $B$ we use the formula

$$
\sum_{i=1}^{j_{h+1}-j_{h}-1} \sum_{j=j_{h}+1}^{j_{h}+i}=\sum_{j=j_{h}+1}^{j_{h+1}-1} \sum_{i=j-j_{h}}^{j_{h+1}-j_{h}-1}
$$

see Figure 7 .

From this observation it follows

$$
B=\sum_{h=0}^{M}\left(q_{j_{h}+1}-q_{j_{h}}\right) \sum_{j=j_{h}+1}^{j_{h+1}-1} s_{j} \sum_{i=j-j_{h}}^{j_{h+1}-j_{h}-1} \frac{x_{j_{h}+1+i}-x_{j_{h}+i}}{x_{j_{h}+1}-x_{j_{h}}} .
$$

From (6.30), (6.32) we get (using again that $q_{j_{0}}=0=q_{j_{M}}$ )

$$
\begin{aligned}
A_{2}+B & =-\sum_{h=0}^{M} \sum_{j=j_{h}+1}^{j_{h+1}-1} s_{j}\left[q_{j_{h}}+\left(q_{j_{h}}-q_{j_{h}+1}\right) \sum_{i=j-j_{h}}^{j_{h+1}-j_{h}-1} \frac{x_{j_{h}+1+i}-x_{j_{h}+i}}{x_{j_{h}+1}-x_{j_{h}}}\right] \\
& =-\sum_{h=0}^{M} \sum_{j=j_{h}+1}^{j_{h+1}-1} s_{j} \widetilde{q}_{j}^{h},
\end{aligned}
$$

where we have set, for $j \notin\left\{j_{h}: h=0, \ldots, M\right\}$,

$$
\widetilde{q}_{j}^{h}:=q_{j_{h}}+\left(q_{j_{h}}-q_{j_{h+1}}\right) \sum_{i=j-j_{h}}^{j_{h+1}-j_{h}-1} \frac{x_{j_{h}+1+i}-x_{j_{h}+1}}{x_{j_{h+1}}-x_{j_{h}}},
$$

which turns out to be equal to (6.22). We conclude that

$$
\langle z, \dot{z}\rangle=-\sum_{h=1}^{M} s_{j_{h}} q_{j_{h}}-\sum_{h=0}^{M} \sum_{j=j_{h}+1}^{j_{h+1}-1} s_{j} \widetilde{q}_{j}^{h}
$$

From (6.33) and $\langle u, \dot{u}\rangle=-\sum_{j=1}^{M} s_{j} q_{j}=-\sum_{h=1}^{M} s_{j_{h}} q_{j_{h}}-\sum_{h=0}^{M} \sum_{j=j_{h}+1}^{j_{h+1}-1} s_{j} q_{j}$ (see (5.99) ) we get

$$
\frac{1}{2} \frac{d}{d \tau}\|u-z\|_{2}^{2}=-\sum_{h=0}^{M} \sum_{j=j_{h}+1}^{j_{h+1}-1} s_{j}\left(q_{j}-\widetilde{q}_{j}^{h}\right)=-\sum_{j=1, \ldots, N, j \neq j_{h}, h=0, \ldots, M} s_{j}\left(q_{j}-\widetilde{q}_{j}^{h}\right),
$$

and this proves (6.20). 
Assume now that there is a constant $0<\lambda<1$ such that (6.23) holds. Then from (6.34) and (6.21) it follows

$$
\frac{d}{d \tau}\|u-z\|_{2}^{2} \leq-r \gamma_{a}(1-\lambda) \sum_{j=1, \ldots, N, j \neq j_{h}, h=0, \ldots, M}\left|s_{j}\right|^{r} .
$$

Define

$$
\omega=\omega_{u}:=s_{0}+\sum_{h=1}^{M} s_{j_{h}} 1_{\left[x_{j_{h}}, \beta[\right.}
$$

It follows

$$
\|u-\omega\|_{2} \leq|I|^{1 / 2} \sum_{j=1, \ldots, N, j \neq j_{h}, h=1, \ldots, M}\left|s_{j}\right| \leq|I|^{1 / 2}\left(\sum_{j=1, \ldots, N, j \neq j_{h}, h=1, \ldots, M}\left|s_{j}\right|^{r}\right)^{1 / r} .
$$

Similarly from (6.29) it follows, for $x_{j_{h}} \leq x<x_{j_{h}+1}$,

$$
\begin{aligned}
z(x) & =\frac{1}{x_{j_{h+1}}-x_{j_{h}}} \sum_{i=0}^{j_{h+1}-j_{h}-1}\left(x_{j_{h}+1+i}-x_{j_{h}+i}\right)\left(\sum_{k=0}^{h} s_{j_{k}}+\sum_{j=0, \ldots, j_{h}+i, j \neq j_{k}, k=0, \ldots, h} s_{j}\right) \\
& =\sum_{k=0}^{h} s_{j_{k}}+\frac{1}{x_{j_{h+1}}-x_{j_{h}}} \sum_{i=0}^{j_{h+1}-j_{h}-1}\left(x_{j_{h}+1+i}-x_{j_{h}+i}\right) \sum_{j=0, \ldots, j_{h}+i, j \neq j_{k}, k=0, \ldots, h} s_{j} .
\end{aligned}
$$

Using (6.38) and the definition (6.36) of $\omega$ it follows

$$
\|z-\omega\|_{2} \leq|I|^{1 / 2} \sum_{j=1, \ldots, N, j \neq j_{h}, h=0, \ldots, M}\left|s_{j}\right| \leq|I|^{1 / 2}\left(\sum_{j=1, \ldots, N, j \neq j_{h}, h=0, \ldots, M}\left|s_{j}\right|^{r}\right)^{1 / r} .
$$

From (6.37) and (6.39) it follows

$$
\|u-z\|_{2}^{r} \leq 2^{r}|I|^{r / 2} \sum_{j=1, \ldots, N, j \neq j_{h}, h=0, \ldots, M}\left|s_{j}\right|^{r} .
$$

From (6.40) and (6.35) we derive

$$
\frac{d}{d \tau}\|u-z\|_{2}^{2} \leq-\gamma_{a} \frac{r(1-\lambda)}{2^{r}|I|^{r / 2}}\|u-z\|_{2}^{r},
$$

which is (6.24).

To conclude the proof it remains to show that we can assume inequality (6.23).

From the definition (6.21) of $q_{l}$ it follows that, if $\kappa$ is the number in Lemma 6.2, then, recalling (6.5),

$$
\frac{\left|q_{j_{h}}\right|}{\left|q_{j}\right|}=\left(\frac{\left|s_{j}\right|}{\left|s_{j_{h}}\right|}\right)^{1-r} \leq\left(\frac{\kappa \sigma}{\left|\sigma_{h}\right|-\kappa \sigma}\right)^{1-r} \leq(2 \kappa)^{1-r}, \quad j \neq j_{h}, h=0, \ldots, M .
$$

Therefore, from the expression (6.22) of $\widetilde{q}_{j}^{h}$ it follows

$$
\left|\widetilde{q}_{j}^{h}\right| \leq 3 \max _{h}\left|q_{j_{h}}\right| \leq 3(2 \kappa)^{1-r}\left|q_{j}\right|, \quad j \neq j_{h}, h=0, \ldots, M .
$$

This shows that, by taking $\kappa$ such that $3(2 \kappa)^{1-r}<\lambda$, we can satisfy condition (6.21). This concludes the proof of the lemma. 


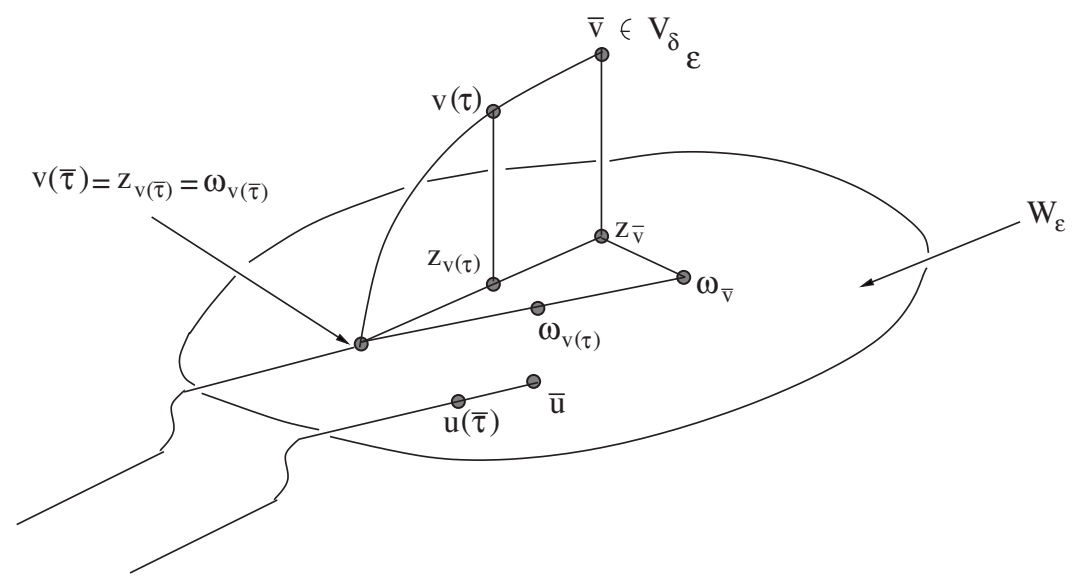

FiguRE 8

We are now in a position to prove Theorem 6.1,

Set $\bar{u}=\bar{\sigma}_{0}+\sum_{h=1}^{M} \bar{\sigma}_{h} 1_{\left[y_{h}, \beta[\right.}$ and $\bar{\sigma}:=\min _{h}\left|\bar{\sigma}_{h}\right|$. Define

$$
W:=\left\{u \in \mathcal{P}_{\text {fin }}(I): \mathcal{H}^{0}\left(J_{u}\right)=\mathcal{H}^{0}\left(J_{\bar{u}}\right)\right\} .
$$

From Theorem 5.4 and the general theory of ODEs, it follows that given $\varepsilon>0$ there exists an $L^{2}$-neighbourhood $W_{\varepsilon} \subset W$ of $\bar{u}$ such that, for each choice of $\bar{w}_{i} \in W_{\varepsilon}$, $i=1,2$, the minimizing movements $w_{i}(\tau)$ starting from $\bar{w}_{i}$ satisfy

$$
\left\|w_{1}(\tau)-w_{2}(\tau)\right\|_{2}<\varepsilon, \quad \tau \in[0,+\infty[.
$$

The main point in the proof is to show (see Figure 8 ) that if

$$
\bar{v} \in V_{\delta}:=\left\{u \in \mathcal{P}_{\text {fin }}(I):\|u-\bar{u}\|_{2}<\delta,|\mathcal{F}(u)-\mathcal{F}(\bar{u})|<\delta\right\},
$$

and $0<\delta \leq \delta_{\varepsilon}$ for some $\delta_{\varepsilon}$, then there exists $\bar{\tau}>0$ such that

$$
\left\{\begin{array}{l}
\|v(\tau)-u(\tau)\|_{2}<\varepsilon \quad \forall \tau \in[0, \bar{\tau}], \\
v(\bar{\tau}), u(\bar{\tau}) \in W_{\varepsilon} .
\end{array}\right.
$$

Once this is proved, to conclude the proof it suffices to identify $v(\bar{\tau}), u(\bar{\tau})$ with $\bar{w}_{1}$, $\bar{w}_{2}$ and $v(\bar{\tau}+s), u(\bar{\tau}+s)$ with $w_{1}(s), w_{2}(s)$.

On the basis of Lemmata 6.2 and 6.3 there exist $\bar{\delta}>0$ and a function $\delta \in] 0, \bar{\delta}[\rightarrow$ $\kappa_{\delta}>0$, with $\lim _{\delta \rightarrow 0^{+}} \kappa_{\delta}=0$, such that

$$
u \in V_{\delta}, \quad u=s_{0}+\sum_{j=1}^{N} s_{j} 1_{\left[x_{j}, \beta[\right.},
$$

implies that the functions $z_{u} \in W, \omega_{u}:=s_{0}+\sum_{h=1}^{M} s_{j_{h}} 1_{\left[x_{j_{h}}, \beta[\right.} \in W$, defined respectively in (6.19), (6.36), are well defined and

$$
\left\|u-z_{u}\right\|_{2}+\left\|u-\omega_{u}\right\|_{2}+\left\|\bar{u}-z_{u}\right\|_{2}+\left\|\bar{u}-\omega_{u}\right\|_{2}=O\left(\kappa_{\delta} \bar{\sigma}\right)=O(\delta),
$$

where $O:] 0, \bar{\delta}[\rightarrow] 0,+\infty\left[\right.$ denotes a function such that $\lim _{s \rightarrow 0^{+}} O(s)=0$.

Moreover we can assume that $\bar{\delta}$ is sufficiently small to ensure that (6.23) and (6.24) hold for any $u \in V_{\bar{\delta}}$. 
Claim 1. There exist $\tau_{1}>0$ and $\left.\delta_{1} \in\right] 0, \bar{\delta} / 2[$ such that

$$
v(\tau) \in V_{\bar{\delta}} \quad \forall \tau \in\left[0, \tau_{1}\left[, \quad \forall \bar{v} \in V_{\delta_{1} / 2}\right.\right.
$$

Thanks to the semicontinuity of $\mathcal{F}$ we can fix $\delta_{1}>0$ such that

$$
\|u-\bar{u}\|_{2}<\delta_{1} \Longrightarrow \mathcal{F}(u) \geq \mathcal{F}(\bar{u})-\frac{\bar{\delta}}{2} .
$$

We have

$$
\begin{aligned}
\|v(\tau)-\bar{v}\|_{2} & \leq \int_{0}^{\tau}\|\dot{v}(t)\|_{2} d t \leq \tau^{1 / 2}\left(\int_{0}^{\tau}\|\dot{v}(t)\|_{2}^{2} d t\right)^{1 / 2} \\
& \leq \tau^{1 / 2}|\mathcal{F}(v(\tau))-\mathcal{F}(\bar{v})|^{1 / 2} \leq \tau^{1 / 2}(\mathcal{F}(\bar{v}))^{1 / 2} \leq \tau^{1 / 2}\left(\mathcal{F}(\bar{u})+\frac{\delta_{1}}{2}\right)^{1 / 2},
\end{aligned}
$$

where the last inequality follows from the assumption $\bar{v} \in V_{\delta_{1} / 2}$. Therefore (6.47) $\|\bar{u}-v(\tau)\|_{2} \leq\|\bar{u}-\bar{v}\|_{2}+\|\bar{v}-v(\tau)\|_{2} \leq \frac{\delta_{1}}{2}+\tau^{1 / 2}\left(\mathcal{F}(\bar{u})+\frac{\delta_{1}}{2}\right)^{1 / 2}<\delta_{1}, \forall \tau \in\left[0, \tau_{1}[\right.$, where $\tau_{1}:=\frac{\delta_{1}^{2}}{2\left(\delta_{1}+2 \mathcal{F}(\bar{u})\right)}$. From ([6.46) and (6.47) we derive $\mathcal{F}(v(\tau)) \geq \mathcal{F}(\bar{u})-\frac{\bar{\delta}}{2}$, that together with $|\mathcal{F}(\bar{u})-\mathcal{F}(\bar{v})|<\frac{\delta_{1}}{2}$ and $\mathcal{F}(\bar{v}) \geq \mathcal{F}(v(\tau))$ implies

$$
|\mathcal{F}(v(\tau))-\mathcal{F}(\bar{u})|<\frac{\delta_{1}}{2}+\frac{\bar{\delta}}{2}<\bar{\delta}, \quad \forall \tau \in\left[0, \tau_{1}[.\right.
$$

From this and (6.47) the claim follows.

Claim 2. There exist $\delta \in] 0, \frac{\delta_{1}}{2}\left[\right.$ and $\tau_{\delta}>0, \tau_{\delta}=O\left(\delta_{1}\right)$, such that

$$
\forall \bar{v} \in V_{\delta} \quad \exists \bar{\tau} \in\left[0, \tau_{\delta}\left[: \quad v(\bar{\tau})=z_{v(\bar{\tau})}=\omega_{v(\bar{\tau})} .\right.\right.
$$

From the previous part of the proof and (6.24) it follows

$$
\frac{d}{d \tau}\left\|v(\tau)-z_{v(\tau)}\right\|_{2}^{2} \leq-C\left(\left\|v(\tau)-z_{v(\tau)}\right\|_{2}^{2}\right)^{r / 2} \quad \forall \tau \in\left[0, \tau_{1}\left[, \bar{v} \in V_{\delta_{1} / 2} .\right.\right.
$$

Let $\delta \in] 0, \delta_{1} / 2[$ be chosen in such a way that

$$
\left\|\bar{v}-z_{\bar{v}}\right\| \leq O(\delta) \leq\left(\frac{C(2-r)}{2} \frac{\tau_{1}}{2}\right)^{\frac{2}{2-r}} .
$$

Then (6.50) and $\|\bar{v}-\bar{u}\|_{2} \leq \delta$ imply

$$
\begin{aligned}
\left\|v(\tau)-z_{v(\tau)}\right\|_{2}^{2} & \leq\left(\left\|\bar{v}-z_{\bar{v}}\right\|_{2}^{2-r}-\frac{C(2-r)}{2} \tau\right)^{\frac{2}{2-r}} \\
& \leq\left(\frac{C(2-r)}{2}\left(\frac{\tau_{1}}{2}-\tau\right)\right)^{\frac{2}{2-r}}, \quad \forall \tau \in\left[0, \tau_{1} / 2\right] .
\end{aligned}
$$

It follows that there exists $0 \leq \bar{\tau} \leq \frac{\tau_{1}}{2}$ such that $\left\|v(\bar{\tau})-z_{v(\bar{\tau})}\right\|_{2}=0$. From this and the expression of $\tau_{1}$ the claim follows with $\tau_{\delta}=\frac{\tau_{1}}{2}$.

From

$$
\|u(\tau)-\bar{u}\|_{2}=O(\bar{\tau})=O\left(\delta_{1}\right), \quad \tau \in[0, \bar{\tau}],
$$

(6.47) and (6.49) it follows

$$
u(\bar{\tau}), v(\bar{\tau}) \in W_{O\left(\delta_{1}\right)} .
$$


From (6.47) and (6.52) we also get

$$
\|u(\tau)-v(\tau)\|_{2}=O\left(\delta_{1}\right), \quad \forall \tau \in[0, \bar{\tau}] .
$$

Therefore there exists $\delta_{1, \varepsilon}>0$ such that $\delta_{1}=\delta_{1, \varepsilon}$ in (6.53), (6.54) implies (6.43). This concludes the proof of the theorem if we define $\delta_{\varepsilon}$ by (6.51) with $\delta_{1}=\delta_{1, \varepsilon}$ and $\tau_{1}$ defined as in (6.47) with $\delta_{1}=\delta_{1, \varepsilon}$.

\section{WEAK SOLUTIONS STARTING FROM $\bar{u}$ WITH INFINITE JUMP POINTS}

Our main result in this section is Theorem 7.4 below, where we consider initial data $\bar{u} \in \operatorname{Dom}(\mathcal{F})$ allowed to have an infinite number of jump points. In this situation we can still associate to $\bar{u}$ a minimizing movement, but we cannot claim uniqueness. To state and prove Theorem 7.4 we need the notions of generalized minimizing movement [22] and descending slope [25], 38], 3].

Definition 7.1. Let $\bar{u} \in L^{2}(I)$ and let $\mathcal{G}$ be as in (5.1). We say that $u:[0,+\infty[\rightarrow$ $L^{2}(I)$ is a generalized minimizing movement associated with $\mathcal{F}$ starting from $\bar{u}$ if there exists a sequence $\left.\left\{\lambda_{i}\right\} \subset\right] 1,+\infty\left[\right.$ and a sequence $w_{i}: \mathbb{N} \cup\{0\} \rightarrow L^{2}(I)$ of functions such that $w_{i}(0)=\bar{u}$ for all $i$ and

(i) $\lim _{i \rightarrow+\infty} \lambda_{i}=+\infty$;

(ii) for each $i \in \mathbb{N}$ and $k \in \mathbb{N} \cup\{0\}$ :

$$
\mathcal{G}\left(\lambda_{i}, w_{i}(k+1), w_{i}(k)\right)=\min \left\{\mathcal{G}\left(\lambda_{i}, v, w_{i}(k)\right): v \in L^{2}(I)\right\} ;
$$

(iii) $\lim _{i \rightarrow+\infty} w_{i}\left(\left[\lambda_{i} \tau\right]\right)=u(\tau)$ in $L^{2}(I)$ for any $\tau \in[0,+\infty[$.

Definition 7.2. Given $v \in \operatorname{Dom}(\mathcal{F})$ we define the descending slope of $\mathcal{F}$ at $v$ as

$$
|\partial \mathcal{F}|(v):=\limsup _{\|w-v\|_{2} \rightarrow 0} \frac{(\mathcal{F}(v)-\mathcal{F}(w), 0)^{+}}{\|v-w\|_{2}}
$$

where $a^{+}:=\max (a, 0)$. We also set

$$
\left|\partial^{-} \mathcal{F}\right|(v):=\inf \left\{\liminf _{h \rightarrow+\infty}|\partial \mathcal{F}|\left(u_{h}\right): u_{h} \rightarrow v\right\}, \quad v \in \operatorname{Dom}(\mathcal{F}),
$$

the relaxed descending slope of $\mathcal{F}$.

Remark 7.3. If $\bar{u} \in \operatorname{Dom}(\mathcal{F})$ and $u$ is a generalized minimizing movement starting from $\bar{u}$, then $\mathcal{F}(u(\tau)) \leq \mathcal{F}(\bar{u})$ for any $\tau \geq 0$, and if $\bar{u} \in L^{\infty}(I)$, then $\|u(\tau)\|_{\infty} \leq$ $\|\bar{u}\|_{\infty}$. If $\bar{u} \in \mathcal{P}_{\text {fin }}(I)$, then the generalized minimizing movement starting from $\bar{u}$ is unique and coincides with the minimizing movement (starting from $\bar{u}$ ) considered in Section 5 ,

Theorem 7.4. Let $\bar{u} \in \operatorname{Dom}(\mathcal{F})$. Then

(i) there exists one generalized minimizing movement u starting from $\bar{u}$.

(ii) The map $\tau \in\left[0,+\infty\left[\rightarrow u(\tau) \in L^{2}(I)\right.\right.$ is absolutely continuous and its metric derivative $\left|u^{\prime}\right|(\tau):=\lim _{h \rightarrow 0} \frac{\|u(\tau+h)-u(\tau)\|_{2}}{|h|}$ belongs to $L^{2}([0,+\infty[)$.

(iii) There exists a nonincreasing function $g:[0,+\infty[\rightarrow \mathbb{R}$ such that

$\frac{1}{2} \int_{\tau_{1}}^{\tau_{2}}\left(\left|u^{\prime}\right|(\tau)\right)^{2} d \tau+\frac{1}{2} \int_{\tau_{1}}^{\tau_{2}}\left(\left|\partial^{-} \mathcal{F}\right|(u(\tau))\right)^{2} d \tau \leq g\left(\tau_{1}\right)-g\left(\tau_{2}\right),\left[\tau_{1}, \tau_{2}\right] \subset[0,+\infty[$. 
(iv) For every $\tau>0$ we have $\mathcal{H}^{0}\left(J_{u(\tau)}\right)<+\infty$. More precisely

$$
\left.\mathcal{H}^{0}\left(J_{u(\tau)}\right)<n \quad \forall \tau \in\right] \tau_{n},+\infty[, \quad \forall n \in \mathbb{N},
$$

where

$$
\tau_{n}:=\frac{C_{\bar{u}}}{n^{2(1-r)}}, \quad C_{\bar{u}}=\frac{2|I|^{4}}{r^{2} \gamma_{a}^{2 / r}}(\mathcal{F}(\bar{u}))^{\frac{2-r}{r}} .
$$

Proof. Assertions (i), (ii) and (iii) are a consequence of Proposition 5.3 and [3, Teorema 2.4]. To prove (iv) we need a careful analysis of $\left|\partial^{-} \mathcal{F}\right|$, that we perform in the next two lemmata.

In the following lemma we show that the domain of the relaxed descending slope coincides with $\mathcal{P}_{\text {fin }}(I)$.

Lemma 7.5. We have

$$
\left|\partial^{-} \mathcal{F}\right|(u)=+\infty \quad \forall u \in \operatorname{Dom}(\mathcal{F}) \backslash \mathcal{P}_{\text {fin }}(I) .
$$

Proof. We first prove that

$$
|\partial \mathcal{F}|(u)=+\infty \quad \forall u \in \operatorname{Dom}(\mathcal{F}) \backslash \mathcal{P}_{\text {fin }}(I) .
$$

Given $u \in \operatorname{Dom}(\mathcal{F}) \backslash \mathcal{P}_{\text {fin }}(I)$, write

$$
u(x)=s_{0}+\sum_{i \in \mathbb{N}: x_{i} \in J_{u}} s_{i} 1_{\left[x_{i}, \beta[\right.}(x),
$$

with $0<\left|s_{i+1}\right| \leq\left|s_{i}\right|$ for all $i \in \mathbb{N}$; since $u \in \operatorname{Dom}(\mathcal{F})$ we have $\sum_{i \in \mathbb{N}}\left|s_{i}\right|^{r}<+\infty$. For $t>0$ and $N \in \mathbb{N}$ define

$$
\varphi_{i}^{N}:= \begin{cases}-\frac{\left|s_{N}\right|^{r}}{t} \operatorname{sign}\left(s_{i}\right)\left|s_{i}\right|^{1-r} & \text { if } i \in\{1, \ldots, N\}, \\ 0 & \text { if } i>N,\end{cases}
$$

and set

$$
\varphi_{N}(x):=\sum_{i \in \mathbb{N}: x_{i} \in J_{u}} \varphi_{i}^{N} 1_{\left[x_{i}, \beta[\right.}(x)
$$

for $x \in I$, and $\varphi_{0}^{N}:=0$. Observe that $0<t\left|\varphi_{i}^{N}\right| \leq\left|s_{i}\right|$ for all $i \in\{1, \ldots, N\}$. Using also the fact that $\left|s_{i}+t \varphi_{i}^{N}\right|=|| s_{i}|-t| \varphi_{i}^{N}||=\left|s_{i}\right|-t\left|\varphi_{i}^{N}\right|$ and the concavity of the function $\xi \in\left[0,+\infty\left[\rightarrow \xi^{r}\right.\right.$, we have

$$
\begin{aligned}
\mathcal{F}(u)-\mathcal{F}\left(u+t \varphi_{N}\right) & =\gamma_{a} \sum_{i=1}^{N}\left(\left|s_{i}\right|^{r}-\left|s_{i}+t \varphi_{i}^{N}\right|^{r}\right) \\
& =\gamma_{a} \sum_{i=1}^{N}\left(\left|s_{i}\right|^{r}-\left(\left|s_{i}\right|-t\left|\varphi_{i}^{N}\right|\right)^{r}\right) \geq \gamma_{a} r t \sum_{i=1}^{N}\left|\varphi_{i}^{N}\right|\left|s_{i}\right|^{r-1} \\
& =\gamma_{a} r\left|s_{N}\right|^{r} \sum_{i=1}^{N}\left|s_{i}\right|^{1-r+r-1}=\gamma_{a} r N\left|s_{N}\right|^{r}
\end{aligned}
$$


Moreover, by Hölder's inequality applied with $p:=(1-r)^{-1}$ and $q=1 / r$, we have

$$
\begin{aligned}
\frac{\left\|\varphi_{N}\right\|_{2}}{|I|^{1 / 2}} & \leq\left\|\varphi_{N}\right\|_{\infty} \leq \sum_{i=1}^{N}\left|\varphi_{i}^{N}\right| \leq N^{r}\left(\sum_{i=1}^{N}\left|\varphi_{i}^{N}\right|^{1 /(1-r)}\right)^{1-r} \\
& =N^{r} \frac{\left|s_{N}\right|^{r}}{t}\left(\sum_{i=1}^{N}\left|s_{i}\right|\right)^{1-r} .
\end{aligned}
$$

From (7.7) and (7.8) we deduce

$$
\frac{\mathcal{F}(u)-\mathcal{F}\left(u+t \varphi_{N}\right)}{t\left\|\varphi_{N}\right\|_{2}} \geq \frac{\gamma_{a} r}{\left(\sum_{i=1}^{N}\left|s_{i}\right|\right)^{1-r}|I|^{1 / 2}} N^{1-r} .
$$

Recalling (2.6) we have $\sum_{i=1}^{N}\left|s_{i}\right| \leq\left(\frac{\mathcal{F}(u)}{\gamma_{a}}\right)^{1 / r}$. Therefore

$$
\frac{\mathcal{F}(u)-\mathcal{F}\left(u+t \varphi_{N}\right)}{t\left\|\varphi_{N}\right\|_{2}} \geq C N^{1-r}
$$

which implies

$$
|\partial \mathcal{F}|(u) \geq C N^{1-r} \quad N \in \mathbb{N}
$$

with

$$
C:=\frac{r \gamma_{a}^{1 / r}}{(\mathcal{F}(u))^{\frac{1-r}{r}}|I|^{1 / 2}},
$$

and (7.5) follows.

We now prove (7.4). Let $\left\{u_{h}\right\} \subset \operatorname{Dom}(\mathcal{F})$ be a sequence converging to $u$ in $L^{2}(I)$; we can assume that $\mathcal{F}\left(u_{h}\right) \leq \mathcal{F}(u)$ for all $h \in \mathbb{N}$. Write

$$
u_{h}=s_{0}^{h}+\sum_{x_{i}^{h} \in J_{u_{h}}} s_{i}^{h} 1_{\left[x_{i}^{h}, \beta[\right.} .
$$

Fix $N \in \mathbb{N}$. Since $\mathcal{H}^{0}\left(J_{u}\right)=+\infty$ it follows that there exists $h_{N}$ such that $\mathcal{H}^{0}\left(J_{u_{h}}\right)>$ $N$ for all $h>h_{N}$. Let $\varphi_{N}^{h}:=\sum_{i} \varphi_{h, i}^{N} 1_{\left[x_{i}^{h}, \beta\right.}$, where $h>h_{N}, \varphi_{h, i}^{N}$ are defined as in (7.6) with $s_{N}^{h}$ and $s_{i}^{h}$ in place of $s_{N}$ and $s_{i}$ respectively. Using (7.10) we have

$$
\frac{\mathcal{F}\left(u_{h}\right)-\mathcal{F}\left(u_{h}+t \varphi_{N}^{h}\right)}{t\left\|\varphi_{N}^{h}\right\|_{2}} \geq C_{h} N^{1-r}, \quad h>h_{N},
$$

where $C_{h}$ is defined as $C$ with $\mathcal{F}\left(u_{h}\right)$ replacing $\mathcal{F}(u)$. Recalling now that $\sup _{h} \mathcal{F}\left(u_{h}\right)$ $\leq \mathcal{F}(u)$, we deduce $C_{h} \geq C$ for all $h$. Hence (7.13) implies $|\partial \mathcal{F}|\left(u_{h}\right) \geq C N^{1-r}$ for all $h>h_{N}$. Therefore $\liminf _{h \rightarrow+\infty}|\partial \mathcal{F}|\left(u_{h}\right) \geq C N^{1-r}$. Since $\left\{u_{h}\right\}$ is an arbitrary sequence converging to $u$, it follows that

$$
\left|\partial^{-} \mathcal{F}\right|(u) \geq C N^{1-r}
$$

Since $N$ is arbitrary, (7.4) follows.

Remark 7.6. From Theorem 7.4 (iii) we deduce that $\mathcal{H}^{0}\left(J_{u(\tau)}\right)<+\infty$ for almost every $\tau \in\left[0,+\infty\left[\right.\right.$. Indeed, if by contradiction $\mathcal{H}^{0}\left(J_{u(\tau)}\right)=+\infty$ for $\tau$ in a set of positive measure in $] 0,+\infty[$, then from Lemma 7.5 we obtain that the second integral on the left hand side of (7.2) is $+\infty$ for some time interval $\left[\tau_{1}, \tau_{2}\right]$, thus contradicting (7.2) itself. 
The following lemma shows that, if $u \in \mathcal{P}_{\text {fin }}(I)$, then $\left|\partial^{-} \mathcal{F}\right|(u)$ coincides with the $L^{2}(I)$-norm of the gradient (at $u$ ) of the restriction $\hat{\mathcal{F}}$ of $\mathcal{F}$ to the set of functions having the same jump points as $u$.

Lemma 7.7. Let $u=s_{0}+\sum_{i=1}^{N} s_{i} 1_{\left[x_{i}, \beta[\right.} \in \mathcal{P}_{\text {fin }}(I), x_{0}:=\alpha<x_{1}<\cdots<x_{N}<$ $x_{N+1}:=\beta$. Then

$$
\left|\partial^{-} \mathcal{F}\right|(u)=|\partial \mathcal{F}|(u)=\|g(u)\|_{2},
$$

where $g=g_{0}+\sum_{i=1}^{N} g_{i} 1_{\left[x_{i}, \beta[\right.}$ is defined in (5.37), (5.38) and (5.39).

Proof. Observe that

$$
-|\partial \mathcal{F}|(u)=\inf \left\{\liminf _{t \rightarrow 0^{+}}\left(\frac{\mathcal{F}(u+t \varphi)-\mathcal{F}(u)}{t}, 0\right)^{-}: \varphi \in \operatorname{Dom}(\mathcal{F}),\|\varphi\|_{2} \leq 1\right\},
$$

where $c^{-}:=\min (c, 0)$. We have

$$
\begin{aligned}
& \frac{\mathcal{F}(u+t \varphi)-\mathcal{F}(u)}{t}=\frac{1}{t} \sum_{x \in J_{\varphi} \backslash J_{u}} \mathrm{e}_{a}\left(t\left|\varphi\left(x_{+}\right)-\varphi\left(x_{-}\right)\right|\right) \\
& +\frac{1}{t} \sum_{x \in J_{\varphi} \cap J_{u}}\left[\mathrm{e}_{a}\left(\left|u\left(x_{+}\right)+t \varphi\left(x_{+}\right)-\left(u\left(x_{-}\right)+t \varphi\left(x_{-}\right)\right)\right|\right)-\mathrm{e}_{a}\left(\left|u\left(x_{+}\right)-u\left(x_{-}\right)\right|\right)\right] .
\end{aligned}
$$

Therefore, since the lim inf as $t \rightarrow 0^{+}$of the first term on the right hand side is $+\infty$, in evaluating the infimum in (7.16) we can restrict the computation to consider only functions $\varphi$ such that $J_{\varphi} \subseteq J_{u}$. We then have

$$
\lim _{t \rightarrow 0^{+}} \frac{\mathcal{F}(u+t \varphi)-\mathcal{F}(u)}{t}=\langle\nabla \hat{\mathcal{F}}(u), \varphi\rangle .
$$

Finally, the equality $\left|\partial^{-} \mathcal{F}\right|(u)=|\partial \mathcal{F}|(u)$ follows from the lower semicontinuity of the right hand side of (7.15).

Remark 7.8. From (7.12), (7.14) we deduce

$$
u \in \mathcal{P}_{\text {fin }}(I) \Longrightarrow \mathcal{H}^{0}\left(J_{u}\right) \leq C_{1}(\mathcal{F}(u))^{1 / r}\left(\left|\partial^{-} \mathcal{F}\right|(u)\right)^{\frac{1}{1-r}},
$$

where $C_{1}:=\left(\frac{1}{r}\right)^{\frac{1}{1-r}} \frac{|I| \frac{2}{1-r}}{\gamma_{a}^{\frac{1}{r(1-r)}}}$.

We are now in a position to conclude the proof of the theorem.

Define $\tau_{n}^{\lambda_{i}}:=\sup \left\{\tau>0: \mathcal{H}^{0}\left(J_{w_{i}\left(\left[\lambda_{i} \tau\right]\right.}\right) \geq n\right\}$. Then by Proposition [5.6] it follows

$$
n \leq \mathcal{H}^{0}\left(J_{w_{i}\left(\left[\lambda_{i} \tau\right]\right)}\right) \quad \forall \tau \in\left[0, \tau_{n}^{\lambda_{i}}\right] .
$$

Therefore by (7.19) and $\mathcal{F}\left(w_{i}\left(\left[\lambda_{i} \tau\right]\right)\right) \leq \mathcal{F}(\bar{u})$ we deduce

$$
n^{1-r} \leq C_{1}^{1-r}(\mathcal{F}(\bar{u}))^{(1-r) / r}\left|\partial^{-} \mathcal{F}\right|\left(w_{i}\left(\left[\lambda_{i} \tau\right]\right)\right) \quad \forall \tau \in\left[0, \tau_{n}^{\lambda_{i}}\right] .
$$

Integrating the square of (7.20) in $] 0, \tau_{n}^{\lambda_{i}}[$ and recalling that

$$
\int_{0}^{\tau_{n}^{\lambda_{i}}}\left(\left|\partial^{-} \mathcal{F}\right|\left(w_{i}\left(\left[\lambda_{i} s\right]\right)\right)\right)^{2} d s \leq 2 \mathcal{F}(\bar{u})
$$

we get

$$
\tau_{n}^{\lambda_{i}} \leq \frac{2 C_{1}^{2(1-r)}(\mathcal{F}(\bar{u}))^{\frac{2-r}{r}}}{n^{2(1-r)}}=\tau_{n}
$$


Therefore

$$
\mathcal{H}^{0}\left(J_{w_{i}\left(\left[\lambda_{i} \tau\right]\right)}\right)<n \quad \forall \tau>\tau_{n}, \forall i \in \mathbb{N} .
$$

Then (7.3) follows from the inequality $\mathcal{H}^{0}\left(J_{u(\tau)}\right) \leq \liminf _{i \rightarrow+\infty} \mathcal{H}^{0}\left(J_{w_{i}\left(\left[\lambda_{i} \tau\right]\right)}\right)$ (see Lemma 5.7). The proof of the theorem is complete.

Remark 7.9. Notice that statement (iv) of Theorem 7.4 also yields upper bounds for the times $\tau_{i}$ in Theorem 5.4

\section{REFERENCES}

[1] G. Alberti, S. Müller: A new approach to variational problems with multiple scales, Comm. Pure Appl. Math. 54 (2001), 761-825. MR1823420 (2002f:49016)

[2] R. Alicandro, A. Braides, M.S. Gelli: Free-discontinuity problems generated by singular perturbations, Proc. Roy. Soc. Edinburgh Sect. A 128 (1998), 1115-1129. MR.1664085 (2000e:35012)

[3] L. Ambrosio: Movimenti minimizzanti, Rend. Acc. Naz. Sc. XL, Mem. Mat. Appl. 113 (1995), 191-246. MR1387558 (97c:49044)

[4] L. Ambrosio, C. De Lellis, C. Mantegazza: Line energies for gradient vector fields in the plane, Calc. Var. Partial Differential Equations, 9 (1999), 227-255. MR1731470(2001g:74021)

[5] L. Ambrosio, N. Fusco, D. Pallara: Functions of Bounded Variation and Free Discontinuity Problems, Clarendon Press (Oxford), 2000. MR1857292 (2003a:49002)

[6] L. Ambrosio, N. Gigli, G. Savaré: Gradient Flows in Metric Spaces and in the Wesserstein Space of Probability Measures, ETH Lectures in Mathematics, Birkhäuser, 2004.

[7] G.I. Barenblatt, M. Bertsch, R. Dal Passo, M. Ughi: A degenerate pseudoparabolic regularisation of a nonlinear forward-backward heat equation arising in the theory of heat and mass exchange in stably stratified turbulent shear flow, SIAM J. Math. Anal. 24 (1993), 1414-1439. MR1241152 (94h:35129)

[8] G.I. Barenblatt, M. Bertsch, R. Dal Passo, V.M. Prostokishin, M. Ughi: A mathematical model of turbulent heat and mass transfer in stably stratified shear flow, J. Fluid Mech. 253 (1993), 341-358. MR1233902 (94g:80005)

[9] G. Bellettini: On gradient flows of some non-convex functionals of Perona-Malik type, Oberwolfach Report 26/2004.

[10] G. Bellettini, G. Fusco: A regularized Perona-Malik functional: Some aspects of the gradient dynamics, EQUADIFF 2003 Proceedings of the International Conference on Differential Equations, Hasselt, Belgium 22 - 26 July 2003, Freddy Dumortier, Henk Broer, Jean Mawhin, Andre Vanderbauwhede and Sjoerd Verduyn Lunel, eds., 639-644. MR2185104

[11] G. Bellettini, G. Fusco, N. Guglielmi: A concept of solution for forward-backward equations of the form $u_{t}=\frac{1}{2}\left(\phi^{\prime}\left(u_{x}\right)\right)_{x}$ and numerical experiments for the singular perturbation $u_{t}=-\epsilon^{2} u_{x x x x}+\frac{1}{2}\left(\phi^{\prime}\left(u_{x}\right)\right)_{x}$, Discrete Contin. Dyn. Systems, 16 (4), 2006, 783-842. MR.2257160 (2007j:35080)

[12] G. Bellettini, M. Novaga, E. Paolini: Global solutions to the gradient flow equation of a nonconvex functional, SIAM J. Math. Anal., 37 (5) (2006), 1657-1687. MR2215602 (2007a:35060)

[13] G. Bouchitté, A. Braides, G. Buttazzo: Relaxation results for some free discontinuity problems, J. Reine Angew. Math. 458 (1995), 1-18. MR1310950 (95j:49014)

[14] G. Bouchitté, C. Dubs, P. Seppecher: Regular approximation of free-discontinuity problems, Math. Mod. Meth. Appl. Sci. 10 (2000), 1073-1097. MR.1780150 (2001k:49033)

[15] A. Braides: Approximation of Free-Discontinuity Problems. Lecture Notes in Math., 1694, Springer 1998. MR:1651773(99j:49001)

[16] A. Braides: $\Gamma$-Convergence for Beginners. Oxford Lecture Series in Mathematics and its Applications, 2002. MR1968440 (2004e:49001)

[17] R.J. Braun, J.W. Cahn, G.B. McFadden, A.A. Wheeler: Anisotropy of interfaces in an ordered alloy: a multiple order parameter model. Trans. Royal Soc. A 355 (1997), 1787-1832. 
[18] F. Catté, P.-L. Lions, J.-M. Morel, T. Coll: Image selective smoothing and edge detection by nonlinear diffusion, SIAM J. Numer. Anal., 29 (1992), 182-193. MR1149092 (93a:94005)

[19] G. CORTESANI: Sequences of non-local functionals which approximate free-discontinuity problems, Arch. Rational Mech. Anal. 144 (1998), 357-402. MR.1656480 (99j:49021)

[20] G. Cortesani, R. Toader: A density result in $S B V$ with respect to non-isotropic energies, Nonlinear Anal. 38 (1999), 1507-1519. MR.1709990 (2000g:49018)

[21] G. DAL MASO: An Introduction to $\Gamma$-convergence, Birkhäuser, Boston (1993). MR 1201152 (94a:49001)

[22] E. De Giorgi: New problems on minimizing movements, In Boundary Value Problems for Partial Differential Equations and Applications, C. Baiocchi and J.-L. Lions, eds., RMA: Res. Notes Appl. Math. 29, Masson, Paris (1993), 81-98. MR.1260440 (95a:35057)

[23] E. De Giongi: Congetture riguardanti alcuni problemi di evoluzione, Duke Math. J. 81 (1995), 255-268

[24] E. De Giongi: Su alcuni problemi instabili legati alla teoria della visione, Atti del Convegno in onore di Carlo Ciliberto (Napoli, 1995), T. Bruno, P. Buonocore, L. Carbone, V. Esposito, eds., 91-98, La Città del Sole, Napoli, 1997.

[25] E. De Giorgi, A. Marino, M. Tosques: Problemi di evoluzione in spazi metrici e curve di massima pendenza, Atti Accad. Naz. Lincei, Rend. Cl. Sci. Fis. Mat. Natur., 68 (1980), 180-187. MR636814 (83m:49052)

[26] S. DeMoulini: Young measure solutions for a nonlinear parabolic equation of forwardbackward type, SIAM J. Math. Anal. 27 (1996), 376-403. MR1377480 (97a:35096)

[27] S. Esedoglu: An analysis of the Perona-Malik scheme, Comm. Pure Appl. Math. LIV (2001), 1442-1487. MR1852979 (2003i:94005)

[28] F. Fierro, R. Goglione, M. Paolini: Numerical simulations of mean curvature flow in the presence of a nonconvex anisotropy, Math. Models Methods Appl. Sci. 8 (1998), 573-601. MR.1634826 (99k:58040)

[29] M. Gobbino: Gradient flow for the one-dimensional Mumford-Shah functional, Ann. Sc. Norm. Sup. Pisa Cl. Sci. (4) XXVII (1998), 145-193. MR1658873 (99j:49003)

[30] M. GobBino: Minimizing movements and evolution problems in Euclidean spaces, Ann. Mat. Pura Appl. CLXXVI (1999), 29-48. MR.1746533 (2001b:49011)

[31] M. Gobbino: Entire solutions of the one-dimensional Perona-Malik equation, Comm. Partial Differential Equations 32 (2007), 719-743.

[32] M.E. Gurtin, H.-M. Soner, P.E. Souganidis: Anisotropic motion of an interface relaxed by the formation of infinitesimal wrinkles, J. Differential Equations, 119 (1995), 54-108. MR1334488 (97a:73013)

[33] K. HöLLIG, Existence of infinitely many solutions for a forward-backward heat equation, Trans. Amer. Math. Soc. 278 (1983), 299-316. MR697076 (84m:35062)

[34] D. Horstmann, H.G. Othmer, K.J. Painter: Aggregation under local reinforcement: From lattice to continuum, European J. Appl. Math. 15 (2004), 546-576. MR2128611 (2006b:35166)

[35] D. Horstmann, B. Schweizer: Comparison of two solution concepts for forward-backward diffusion, Preprint (2006).

[36] B. KaWohl, N. Kutev: Maximum and comparison principle for one-dimensional anisotropic diffusion, Math. Ann. 311 (1998), 107-123. MR1624275 (99h:35110)

[37] S. Kichenassamy: The Perona-Malik paradox, SIAM J. Appl. Math. 57 (2001), 1328-1342. MR.1470926 (98i:35088)

[38] A. Marino, C. Saccon, M. Tosques: Curves of maximal slope and parabolic variational inequalities on nonconvex constraints, Ann. Scuola Norm. Sup. Pisa Cl. Sci. (4) 16 (1989), 281-330. MR1041899 (91d:58035)

[39] M. Morini: Sequences of singularly perturbed functionals generating free-discontinuity problems, SIAM J. Math. Anal. 35, (2003), 759-805. MR2048406 (2005a:49023)

[40] S. MüLLER: Variational models for microstructure and phase transitions, Calculus of variations and geometric evolution problems (Cetraro 1996), 85-210, Lecture Notes in Math., 1713, Springer, Berlin, 1999. MR1731640 (2001b:49019) 
[41] M. Nitzberg, D. Mumford, T. Shiota: Filtering, Segmentation and Depth, Lecture Notes in Computer Science, vol. 662, Springer-Verlag, Berlin, 1993. MR.1226232 (94h:92012)

[42] K.J. Painter, D. Horstmann, H.G. Othmer: Localization in lattice and continuum models of reinforced random walks, Appl. Math. Lett. 16 (2003), 375-381. MR1961428

[43] P. Perona, J. Malik: Scale space and edge detection using anisotropic diffusion, IEEE Trans. Pattern Anal. Mach. Intell. 12 (1990), 629-639.

[44] P.I. Plotnikov: Passage to the limit with respect to viscosity in an equation with variable parabolicity direction, Differential Equations 30 (1994), 614-622. MR.1299852 (95g:35085)

[45] M. Slemrod: Dynamics of measure valued solutions to a backward-forward heat equation, J. Dynam. Differential Equations 3 (1991), 1-28. MR1094722 (92m:35124)

Dipartimento di Matematica, Università di Roma "Tor Vergata", via della Ricerca Scientifica 00133 Roma, Italy - And - INFN Laboratori NaZionali di Frascati, Italy

E-mail address: belletti@mat.uniroma2.it

Dipartimento di Matematica, Università di L'Aquila, via Vetoio, loc. Coppito, 67100 L'Aquila, ITALy

E-mail address: fusco@univaq.it 